\title{
Index Theory and Positive Scalar Curvature
}

\author{
Dissertation \\ zur Erlangung des mathematisch-naturwissenschaftlichen Doktorgrades \\ "Doctor rerum naturalium" \\ der Georg-August-Universität Göttingen
}

\author{
vorgelegt von \\ Daniel Pape
}

aus Stadtoldendorf

Göttingen 2011 


\section{Referent:}

Prof. Dr. Thomas Schick

\section{Koreferent:}

Prof. Dr. Bernhard Hanke

Mitglieder der Prüfungskommission:

Prof. Dr. Bernhard Hanke

Prof. Dr. Preda Mihailescu

Prof. Dr. Thomas Schick

Prof. Dr. Max Wardetzky

Prof. Dr. Ingo Witt

Prof. Chenchang Zhu, Ph.D.

Tag der mündlichen Prüfung:

23.09.2011 
In Erinnerung an Hans Kick 



\section{Contents}

0. Introduction $\quad 1$

0.1 . The problem ..................... 1

0.2 . Presentation of results . . . . . . . . . . . 2

0.3. Organization of this thesis . . . . . . . . . . . . 4

$\begin{array}{ll}\text { Acknowledgements } & 5\end{array}$

1. Coarse index theory and positive scalar curvature 6

1.1. Introduction . . . . . . . . . . . . . . . 6

1.2. The coarse $\mathrm{C}^{*}$-algebra $\ldots \ldots \ldots \ldots \ldots \ldots$

1.3. Coarse indices . . . . . . . . . . . . . . . . . . 11

1.3.1. The functional calculus for regular operators . . . . . . . 12

1.3.2. Definition of the coarse indices . . . . . . . . . . 20

1.4. A vanishing theorem for the coarse indices . . . . . . . . . . 23

1.4.1. Introduction . . . . . . . . . . . . 23

1.4.2. Finite-dimensionality of the $L^{2}$-kernel . . . . . . . . . 24

1.4.3. Preliminary facts about Hilbert $\mathrm{C}^{*}$-modules . . . . . . 27

1.4.4. The vanishing of $\mathrm{K}_{0}(i)$ : First proof . . . . . . . . 32

1.4.5. The vanishing of $\mathrm{K}_{0}(i)$ and $\mathrm{K}_{1}(i)$ : General proof. . . . . . 37

1.4.6. The vanishing theorem . . . . . . . . . . . . . 40

1.5. Roe's partitioned manifold index theorem . . . . . . . . . . 45

1.5.1. Introduction . . . . . . . . . . . . 45

1.5.2. Dirac operators on hypersurfaces . . . . . . . . 45

1.5.3. The theorem . . . . . . . . . . . . 51

1.6. A geometric application . . . . . . . . . . . . 56

2. A counterexample to a conj. about pos. scalar curvature $\quad 65$

2.1. Introduction . . . . . . . . . . . . . 65

2.2. Preliminary remarks . . . . . . . . . . . . 65

2.3. The counterexample . . . . . . . . . . . . 66

$\begin{array}{ll}\text { A. Appendix } & \mathbf{7 0}\end{array}$

1.1. Some technical lemmas . . . . . . . . . . . . . . . . 70

1.2. More detailed estimates . . . . . . . . . . . . 73

$\begin{array}{ll}\text { Conventions } & 74\end{array}$

$\begin{array}{ll}\text { Nomenclature } & 74\end{array}$

$\begin{array}{ll}\text { References } & 74\end{array}$ 


\section{Introduction}

\subsection{The problem}

A major open problem in differential geometry is to decide whether a given smooth manifold admits a Riemannian metric of positive scalar curvature or not. This question has attracted a lot of attention over the last decades since it is both mathematically intriguing as well as important for applications in the realm of mathematics and physics. We only name Seiberg-Witten theory and the Einstein-Hilbert action here.

Let $M$ be a smooth (compact or non-compact) manifold (with or without) boundary. As stated above the central problem we are interested in within this thesis is whether or not $M$ admits a Riemannian metric $g_{i j}$ whose scalar curvature

$$
\kappa=\sum g^{i j} R_{i j k}^{k}
$$

is an every positive function. Each sphere $S^{n}$ (for $n \in \mathbb{N}_{\geq 2}$ ) and each (real, complex or quaterionic) projective space admits a Riemannian metric of positive scalar curvature. The same is true for each compact semi-simple Lie group. By [15] no torus $T^{n}$ (for $n \in \mathbb{N}$ ) admits a Riemannian metric of positive scalar curvature. It is well-known that each compact manifold with $\operatorname{dim}(M) \geq 3$ and each complete non-compact manifold with $\operatorname{dim}(M) \geq 5$ admits a metric of constant negative scalar curvature, see [25] and [4]. The existence of a Riemannian metric of positive scalar curvature on the other hand is non-trivially linked to the topology of the manifold. The first important result in this direction, due to Lichnerowicz, shows that the $A$-hat-genus $\hat{A}(M)$ of a closed spin manifold $M$ must vanish in order for the manifold to admit a metric of positive scalar curvature. Here the $A$-hat genus is a topological datum of the manifold defined via the Pontryagin classes of $M$ and Hirzebruch's calculus of multiplicative sequences. This result is obtained by means of the Atiyah-Singer index theorem and Lichnerowicz' astute observation that the square of the Dirac operator induced by the spin structure satisfies the equation

$$
D^{2}=\nabla^{*} \nabla+\frac{\kappa}{4} \text { id } .
$$

This interplay between index theory of Dirac-type operators and positive scalar curvature was refined in later years and accumulated in the Gromov-LawsonRosenberg Conjecture which gives both a necessary and sufficient condition for a compact spin manifold (with $\operatorname{dim}(M) \geq 5$ ) to admit a metric of positive scalar curvature, and links this question to the K-theory of the group $\mathrm{C}^{*}$-algebra of the fundamental group $\pi=\pi_{1}(M)$ of $M$. This conjecture is known to be true in many cases but also known to fail in general, see [24] or [41]. 


\section{Introduction}

\subsection{Presentation of results}

This thesis has two main results, which we set out to describe next.

The first result is a vanishing theorem for coefficient-versions of the higher Roe indices, and is stated as Theorem 1.4.28 in Subsection 1.4.6. It gives a generalization of a result previously obtained by Roe [37] and $\mathrm{Yu}$ [49]. A similar result was obtained by Block and Weinberger [5] based on previous work by Bunke [6]. After stating the result below we compare it briefly with the one of Block and Weinberger. Our result reads as follows.

0.2.1 Theorem (Vanishing theorem). Let $(M, g)$ be non-compact complete Riemannian spin manifold whose scalar curvature is uniformly positive outside of a compact subset. Then the even (if $\operatorname{dim}(M)$ is even) and odd Roe index with coefficients in the group $\mathrm{C}^{*}$-algebra $A=\mathrm{C}^{*} \pi$ of the fundamental group $\pi=\pi_{1}(M)$

$$
\operatorname{ind}_{0}(D) \in \mathrm{K}_{0}\left(\mathrm{C}^{*}(M, A)\right)
$$

and

$$
\operatorname{ind}_{1}(D) \in \mathrm{K}_{1}\left(\mathrm{C}^{*}(M, A)\right)
$$

vanish. Here $D: \Gamma(M, S) \rightarrow \Gamma(M, S)$ with $S=\Sigma M \otimes \mathscr{V}_{M}$ is the twisted Dirac operator obtained by twisting the spinorial Dirac operator of $(M, g)$ with the Mishchenko line bundle $\left(\mathscr{V}_{M}, \nabla^{\mathscr{V} M}\right)$.

As indicated above this result was previously known for the untwisted spinorial Dirac operator and the usual Roe indices (i.e. those with complex coefficients).

Our vanishing theorem bears similarities to the Bochner-Lichnerowicz theorem of Block and Weinberger [5, Thm. 4.8] which reads as follows.

0.2.2 Theorem (see [5]). Let $(M, g)$ be an $n$-dimensional complete Riemannian spin manifold with fundamental group $\pi=\pi_{1}(M)$. Let $f: M \rightarrow \mathbb{R}$ be a smooth and proper map and $N=f^{-1}(t)$ a regular submanifold (compact and of codimension 1) corresponding to a regular value $t \in \mathbb{R}$. If the scalar curvature of the Riemannian manifold is uniformly positive outside a compact subset, the higher index à la Rosenberg

$$
\operatorname{ind}_{\mathrm{C}_{r}^{*} \pi}\left(D_{N} \otimes\left(\mathscr{V}_{M}\right)_{\mid N}\right)=\left[C_{t} \otimes f * \otimes C_{M}\right] \in \mathrm{K}_{n-1}\left(\mathrm{C}_{r}^{*} \pi\right)
$$

vanishes. (That this index is described by a certain correspondence $C_{t} \otimes f * \otimes C_{M}$ in the sense of Connes and Skandalis is the content of [5, Thm. 3.4].) Notice that the given index is not the usual Rosenberg $\alpha$-index $\alpha(N):=$ $\operatorname{ind}_{\mathrm{C}_{r}^{*} \pi_{1}(N)}\left(D_{N} \otimes \mathscr{V}_{N}\right)$ lying in $\mathrm{K}_{*}\left(\mathrm{C}^{*} \pi_{1}(N)\right)$.

This result is referred to as 'equivariant version of Roe's partitioned index theorem' in [8, p. 7]. Indeed, it is pointed out in [5, p. 389] that the theorem can be reduced to Roe's partitioned manifold index theorem when $\pi$ is trivial. We note that though both results assume that the scalar curvature is positive at infinity, the manifold $M$ in our vanishing theorem has not to be 'partitioned' by a codimension one submanifold $N$. Furthermore, the considered indices lie in different K-theory groups. 


\section{Introduction}

The partitioned manifold index theorem by Roe has recently been extended by Zadeh [51] to a coefficient version. As an application of our vanishing result, one can obtain a codimension two obstruction result similar to the one given by Gromov and Lawson [16, Thm. 7.47] using Zadeh's version of Roe's theorem.

Our vanishing theorem implies together with the coefficient version of Roe's index theorem the following result which the reader should compare with the Bochner-Lichnerowicz theorem of Block and Weinberger stated above.

0.2.3 Theorem. Let $(M, g)$ be an $n$-dimensional complete Riemannian spin manifold with fundamental group $\pi=\pi_{1}(M)$. Let $f: M \rightarrow \mathbb{R}$ be a smooth and proper map and $N=f^{-1}(t)$ a regular submanifold (compact and of codimension 1) corresponding to a regular value $t \in \mathbb{R}$. If the scalar curvature of the Riemannian manifold is uniformly positive outside a compact subset the Rosenberg $\alpha$-index of $N$

$$
\alpha(N)=\operatorname{ind}_{\mathrm{C}^{*} \pi_{1}(N)}\left(D_{N} \otimes \mathscr{V}_{N}\right) \in \mathrm{K}_{n-1}\left(\mathrm{C}^{*} \pi_{1}(N)\right)
$$

vanishes, provided there exists a flat bundle $E$ over $M$ with $E_{\mid N}=\mathscr{V}_{N}$. More generally, we have: If $E$ is a flat bundle over $M$, then the index of $D_{M} \otimes E$ is related to the index of $D_{N} \otimes E_{\mid N}$ by

$$
\zeta_{N}\left(\operatorname{ind}_{\mathrm{C}^{*} \pi_{1}(M)}\left(D_{M} \otimes E\right)\right)=\operatorname{ind}_{\mathrm{C}^{*} \pi_{1}(N)}\left(D_{N} \otimes E_{\mid N}\right)
$$

where

$$
\zeta_{N}: \mathrm{K}_{n}\left(\mathrm{C}^{*}\left(M, \mathrm{C}^{*} \pi\right)\right) \rightarrow K_{n-1}\left(\mathrm{C}^{*} \pi_{1}(N)\right)
$$

is a certain homomorphism induced by $f$, constructed in Subsection 1.5.3.

Thus the vanishing of $\operatorname{ind}_{\mathrm{C}^{*} \pi_{1}(M)}\left(D_{M} \otimes E\right)$ (following from Theorem 0.2.1) is the reason for the vanishing of $\alpha(N)$. This can sometimes be used to conclude from the non-existence of a Riemannian metric of positive scalar curvature on a submanifold the non-existence of such a metric on the ambient manifold. The codimension two obstruction theorem of Section 1.6 is such an application.

The second result is a counterexample to a recent conjecture from [7] giving necessary and sufficient conditions for the existence of a Riemannian metric of positive scalar curvature on so-called (compact) totally non-spin manifolds. This result is stated as Proposition 2.3.3 in Section 2.3 of Chapter 2. Here a totally non-spin manifold is one for which neither the manifold nor its universal covering admits a spin structure. Nevertheless the manifolds considered by the conjecture are assumed to be at least orientable, and it reads as follows.

0.2.4 Conjecture (see [7]). Suppose that $M$ is a compact oriented totally nonspin manifold, with fundamental group $\pi$ and of dimension $n \geq 5$. Let $f: M \rightarrow$ $\underline{B} \pi$ be the composition of the classifying map $c: M \rightarrow B \pi$ of the universal covering of $M$, and the natural map $B \pi \rightarrow \underline{B} \pi$. Denote by $[M]$ the fundamental class of $M$ in $H_{n}(M)$. Then $M$ admits a metric of positive scalar curvature if and only if $f_{*}[M]$ vanishes in $H_{n}(\underline{B} \pi)$.

Here $B \pi$ is the classifying space of the group $\pi$ and $\underline{B} \pi$ is the (maybe less familiar) classifying space for proper actions. 


\subsection{Organization of this thesis}

The whole work is divided in two chapters, each chapter bringing one of the two main results announced in the previous section. Finally, there is a brief appendix with auxiliary results used in the proof of Theorem 1.4.27.

The first chapter deals with the vanishing theorem for the coarse indices. We begin in Section 2 by briefly recapitulating the definition of the coarse $\mathrm{C}^{*}$ algebra (with coefficients) and of a relative version of this algebra, which will be used in the proof of the vanishing theorem. In Section 3 we proceed to first discuss Kucerovsky's functional calculus [28] for regular operators on Hilbert $\mathrm{C}^{*}$-modules which is then used to define coefficient version of the coarse indices in a second step. In the final part of Section 3 these are shown in the usual way to be obstructions to the existence of a metric of uniform positive scalar curvature and thus are suitable to investigate the existence of metrics of positive scalar curvature via covering spaces. Section 4 contains the vanishing theorem and is the central part of the first chapter. The proof relies on two auxiliary results. The first is contained in Subsection 1.4.4 and 1.4.5 and shows that the inclusion of the compact operators into the Roe $\mathrm{C}^{*}$-algebra induces the trivial map in K-theory. This is proven for even and odd K-theory in Subsection 1.4.5 and in a different way for even K-theory in the preceding Subsection 1.4.4. Though the proof of the former is more general the last is more illustrative. The second auxilliary result is contained in Subsection 1.4.6 and roughly states that some normalized function of the twisted spinorial Dirac operator is a compact operator if the scalar curvature is positive at infinity. Chapter 1 concludes with a discussion of the coefficient version of Roe partitioned manifold index theorem and the derivation of a geometric application of the vanishing theorem in the final Sections 5 and 6, respectively.

The second chapter is rather brief and contains after an introduction and some preparatory remarks the above announced counterexample 


\title{
Acknowledgements
}

\author{
In irgendeinem abgelegenen Winkel des in \\ zahllosen Sonnensystemen flimmernd aus- \\ gegossenen Weltalls gab es einmal ein Ge- \\ stirn, auf dem kluge Tiere das Erkennen er- \\ fanden. Es war die hochmütigste und ver- \\ logenste Minute der $\gg$ Weltgeschichte $\ll$ : aber \\ doch nur eine Minute. Nach wenigen Atem- \\ zügen der Natur erstarrte das Gestirn, \\ und die klugen Tiere mussten sterben. \\ Friedrich Nietzsche, Über Wahrheit \\ und Lüge im außermoralischen Sinne, 1873
}

To begin with, I would like to thank all those who have accompanied me on my way over the last past years to where I stand today. I am especially grateful to my adviser Thomas Schick for always taking the time to discuss with me, for answering my questions patiently and sharing his mathematical insights with me. His guidance has been truely exceptional and I owe many thanks to him. I am also grateful to Bernhard Hanke for expressing his willingness to be the co-marker for this work.

Furthermore, I would like to express my gratitude to my parents and grandparents for supporting me over the past years. My brother has always been there for me, and I would like to thank him for this. I am deeply indebted to Antje Nücklich for all she ever did for me, and for being such a wonderful friend.

I would like to thank Henrik Schumacher and Ingo Treunowski who helped me to sort out the worst blunders by proof-reading parts of this thesis, and Tathagata Banerjee as well as Holger Kammeyer for skimming through several pages.

Another thank you goes to Nicolas Ginoux, Bernhard Hanke, Ralf Meyer, and Mostafa Zadeh for numerous emails. Mostafa Zadeh also shared preprint versions of his articles with me.

Finally, I would like to acknowledge financial support by the Deutsche Forschungsgemeinschaft (DFG) and extend my thanks to everyone in the RTG 1493 and at the Mathematical Institute for making Göttingen such a unique place. 


\section{Coarse index theory and positive scalar curvature}

\subsection{Introduction}

The main objective of this chapter is to formulate and prove a vanishing theorem for the coarse indices of certain Dirac operators. More specifically, the class of operators we study consists of twisted spinorial Dirac operators on complete Riemannian spin manifolds where the twisting bundle is the Mishchenko line bundle of the underlying spin manifold. In coarse index theory one studies certain higher indices of Dirac operators like these. These higher indices take values in the $\mathrm{K}$-theory of the coarse $\mathrm{C}^{*}$-algebra (or Roe $\mathrm{C}^{*}$-algebra) associated with the metric structure of the underlying Riemannian manifold. The term 'coarse' is used because the assignment of a Riemannian manifold (or more generally a proper metric space) to the $\mathrm{K}$-theory of its associated coarse $\mathrm{C}^{*}$ algebra becomes functorial with respect to so-called coarse maps which appear in the study of the large scale geometry of these spaces. For a more detailed account of coarse index theory and Roe's coarse geometry we refer the interested reader to [21], [37], [38] and [40].

In Section 2 of this chapter we introduce the coarse $\mathrm{C}^{*}$-algebra associated with a representation $\rho: C_{0}(X) \rightarrow \mathscr{L}_{A}^{*}(\mathscr{H})$ of the $\mathrm{C}^{*}$-algebra of continuous functions on a metric space $X$ vanishing at infinity by adjointable operators on a Hilbert $A$-module. In this kind of generality the notion of a coarse $\mathrm{C}^{*}$-algebra already appeared in [20]. After introducing a relative version of the coarse $\mathrm{C}^{*}$-algebra which will be important in the course of the proof of the targeted vanishing theorem, we introduce the coarse indices in Section 3. For this purpose we briefly recall the notion of a regular operator on a Hilbert $\mathrm{C}^{*}$-module and describe Kucerovsky's [28] functional calculus for these operators. After this we can define the coarse indices and show that they are obstructions to the existence of uniform positive scalar curvature metrics. In the following Section 4 we state the announced vanishing theorem. This will generalize a previously known version for the case $A=\mathbb{C}$, which is due to Roe [37] and $\mathrm{Yu}$ [49]. The original proofs of Roe and $\mathrm{Yu}$ are based on the finite dimensionality of the $L^{2}$-kernel of the spinorial Dirac operators on a complete Riemannian spin manifold whose metric has positive scalar curvature outside of a compact subset. We briefly review this result of Gromov and Lawson [16] in Subsection 1.4.2. After some preparation in Subsection 1.4.3, we show in Subsection 1.4.4 that the map induced by the inclusion of the compact operators in the Roe $\mathrm{C}^{*}$-algebra vanishes in even K-theory. An analogous result holds for the odd K-theory as we show in Subsection 1.4.5 by means of a more general result, which applies both to 
the even and odd case. The generalized version of the vanishing theorem will be given in Subsection 1.4.6. In the following final parts of this chapter we recall Roe's partitioned manifold index theorem in Section 5 and describe in Section 6 how this theorem together with the vanishing theorem can be used to prove a codimension two-obstruction theorem in style of the one by Gromov and Lawson [16, Thm. 7.47].

\subsection{The coarse $C^{*}$-algebra}

In this section we introduce modules over metric spaces and their associated coarse $\mathrm{C}^{*}$-algebra. We will assume that the reader is familiar with the notion of a Hilbert $\mathrm{C}^{*}$-module. An introduction (and much more) to Hilbert $\mathrm{C}^{*}$-modules can be found in [34], [43], [44] or [48].

1.2.1 Notation. If $A$ is a $\mathrm{C}^{*}$-algebra and $\mathscr{H}$ a Hilbert $A$-module, we denote by $\mathscr{L}_{A}^{*}(\mathscr{H})$ and $\mathscr{K}_{A}(\mathscr{H})$ the $\mathrm{C}^{*}$-algebra of all adjointable operators and of all compact operators $^{1}$ on $\mathscr{H}$, respectively. Likewise, $\mathscr{U}_{A}(\mathscr{H})$ denotes the set of all unitary operators on $\mathscr{H}$. For $S, T \in \mathscr{L}_{A}^{*}(\mathscr{H})$ we write $S \sim T$ if $S-T \in \mathscr{K}_{A}(\mathscr{H})$.

Let $X$ be a locally compact ${ }^{2}$ metric space and $\mathscr{H}$ a Hilbert $A$-module, where $A$ is a unital (complex) $\mathrm{C}^{*}$-algebra. We introduce the following language: An $X$ module structure for $\mathscr{H}$ is a $*$-homomorphism $\rho: C_{0}(X) \rightarrow \mathscr{L}_{A}^{*}(\mathscr{H})$, i.e. a representation of the $\mathrm{C}^{*}$-algebra $C_{0}(X)$ by adjointable operators on $\mathscr{H}$. An $X$ module is a pair $(\mathscr{H}, \rho)$ consisting of a Hilbert $A$-module $\mathscr{H}$ and an $X$-module structure $\rho$ for $\mathscr{H}$.

The following is our main example.

1.2.2 Example. Let $X=M$ be an oriented Riemannian manifold, $A$ a unital $\mathrm{C}^{*}$-algebra, and $\mathscr{H}=L^{2}(M, E)$ the Hilbert $A$-module of squareintegrable sections of a bundle $\pi: E \rightarrow M$ of Hilbert $A$-modules (here integration is with respect to the Riemannian-Lebesgue measure given by the metric and the orientation). This becomes an $X$-module via the $*$-homomorphism $\rho: C_{0}(X) \rightarrow \mathscr{L}_{A}^{*}(\mathscr{H})$ for which $\rho(\varphi)=M_{\varphi}$ is the operator given by multiplying sections of $E$ by $\varphi$. We will often use $\rho(\varphi)$ in this sense for $\varphi \in C(X)$ not necessarily in $C_{0}(X)$.

1.2.3 Remark. Sometimes it is useful to impose additional requirements on $\rho$, such as for example that $\rho$ should be non-degenerate (i.e. the image of the action is dense) and ample (i.e. no non-zero element acts via $\rho$ as a compact operator). These conditions are fulfilled in Example 1.2.2 and can be used to make the assignment of a metric space $X$ to the K-theory of its coarse $\mathrm{C}^{*}$ algebra (to be introduced below) functorial by introducing the notion of 'coarse maps' between such spaces. Cf. [21, Def. 5.1.3] and [21, p. 149 f.], or [38, p. 19].

\footnotetext{
${ }^{1}$ See Definition 1.4.10 later in this chapter.

${ }^{2}$ We assume this because we want that $C_{0}(X)$ is $\mathrm{C}^{*}$-algebra. In addition to this, this assumption will allow us to construct cut-off functions later on.
} 
1. Coarse index theory and positive scalar curvature

1.2.4 Notation. Points in $X$ are denoted $x, y$, z, etc. Elements of $C_{0}(X)$ are denoted by $\varphi, \psi$, etc. Elements of $\mathscr{H}$ are denoted $u, v$, etc.

1.2.5 Definition. Let $(\mathscr{H}, \rho)$ be an $X$-module.

(i) The support of an element $u \in \mathscr{H}$, denoted by $\operatorname{supp}(u)$, is the complement in $X$ of the following subset

$$
\bigcup\left\{U \subset X ; U \text { is open and } \forall \varphi \in C_{0}(U): \rho(\varphi) u=0\right\} .
$$

(ii) The support of an operator $T \in \mathscr{L}_{A}^{*}(\mathscr{H})$, denoted by $\operatorname{supp}(T)$, is the complement in $X \times X$ of the following subset

$$
\left\{(x, y) \in X \times X ; \exists \varphi, \psi \in C_{0}(X) \text { with } \varphi(x), \psi(y) \neq 0: \rho(\varphi) T \rho(\psi)=0\right\} \text {. }
$$

Denote by $\mathscr{H}_{\text {cpt }}$ the set of all compactly supported elements in $\mathscr{H}$.

(iii) An operator $T \in \mathscr{L}_{A}^{*}(\mathscr{H})$ is pseudolocal if $[T, \rho(\varphi)] \in \mathscr{K}_{A}(\mathscr{H})$ holds for any $\varphi \in C_{0}(X)$. The $\mathrm{C}^{*}$-algebra of all pseudolocal operators is denoted by $\Psi^{0}(X)$.

(iv) An operator $T \in \mathscr{L}_{A}^{*}(\mathscr{H})$ is locally compact if both $\rho(\varphi) T \in \mathscr{K}_{A}(\mathscr{H})$ and $T \rho(\varphi) \in \mathscr{K}_{A}(\mathscr{H})$ hold for any $\varphi \in C_{0}(X)$. The $\mathrm{C}^{*}$-algebra of all locally compact operators is denoted by $\Psi^{-1}(X)$.

(v) An operator $T \in \mathscr{L}_{A}^{*}(\mathscr{H})$ has finite propagation ${ }^{3}$ if there exists $R>0$ such that $\rho(\varphi) T \rho(\psi)=0$ for all $\varphi, \psi \in C_{0}(X)$ with $d(\operatorname{supp}(\varphi), \operatorname{supp}(\psi)) \geq$ $R$. The infimum over all such $R$ is called the propagation of $T$.

(vi) Call $(\mathscr{H}, \rho)$ admissible if $\mathscr{H}_{\mathrm{cpt}}$ is dense in $\mathscr{H}$, and one has $\rho(\varphi) u=u$, whenever $u \in \mathscr{H}_{\mathrm{cpt}}$ and $\varphi \in C(X)$ with $\varphi_{\mid \operatorname{supp}(u)}=1$. Furthermore, we require $u=0$ if and only if $\operatorname{supp}(u)=\varnothing$.

One readily verifies that $\Psi^{-1}(X)$ is a $\mathrm{C}^{*}$-ideal in $\Psi^{0}(X)$.

1.2.6 Notation. We will denote the set of all locally compact operators $T \in$ $\Psi^{-1}(X)$ which are of finite propagation by $\Psi_{\mathrm{fp}}^{-1}(X)$.

The next lemma gives another characterization of finite propagation operators.

1.2.7 Lemma. Let $X$ be a locally compact metric space and $(\mathscr{H}, \rho)$ an admissible $X$-module. The following are equivalent:

(i) The operator $T \in \mathscr{L}_{A}^{*}(\mathscr{H})$ has finite propagation.

(ii) There exists $R>0$ such that $\operatorname{supp}(T u) \subset \overline{B(\operatorname{supp}(u) ; R)}$ holds for every element $u \in \mathscr{H}$.

\footnotetext{
${ }^{3}$ Finite propagation operators are also often called controlled operators or operators of bounded propagation.
} 
Proof. "(i) $\Longrightarrow$ (ii)": Let $R>0$ be as in (v) of Definition 1.2.5. We show that $x \notin \overline{B(\operatorname{supp}(u) ; R)}$ implies

$$
x \in \bigcup\left\{U \subset X ; U \text { is open and } \forall \varphi \in C_{0}(U): \rho(\varphi) u=0\right\} .
$$

By assumption, there exists a small $\varepsilon>0$ such that $x \in U$ where $U$ is the open set $U:=X \backslash \overline{B(\operatorname{supp}(u) ; R+\varepsilon)}$. Furthermore, one has for any $\varphi \in C_{0}(U)$

$$
\rho(\varphi) T u=\rho(\varphi) T \rho(\psi) u=0 .
$$

Here $\psi \in C_{0}(X)$ is a function with $\psi_{\mid \operatorname{supp}(u)}=1$ and $\psi_{\mid X \backslash B(\operatorname{supp}(u) ; \varepsilon)}=0$. Such a function $\psi$ exists by [30, Section 2.4] since $X$ is locally compact, and $\rho(\varphi) T \rho(\psi)=0$ holds because $d(\operatorname{supp}(\varphi), \operatorname{supp}(\psi)) \geq R$.

"(ii) $\Longrightarrow$ (i)": Let $u \in \mathscr{H}$ and $\varphi, \psi \in C_{0}(M)$. Then one has for $R>0$ as in (ii)

$$
\begin{aligned}
\operatorname{supp}((\rho(\varphi) T \rho(\psi)) u) & \subset \operatorname{supp}(\varphi) \cap \operatorname{supp}((T \rho(\psi)) u) \\
& \subset \operatorname{supp}(\varphi) \cap \overline{B(\operatorname{supp}(\psi) \cap \operatorname{supp}(u) ; R)} \\
& \subset \operatorname{supp}(\varphi) \cap \overline{B(\operatorname{supp}(\psi) ; R)}
\end{aligned}
$$

and the set on the RHS is empty. Thus $\operatorname{supp}((\rho(\varphi) T \rho(\psi)) u)=\varnothing$, which implies $\rho(\varphi) T \rho(\psi)=0$ by (vi) of Definition 1.2.5.

We now introduce the $\mathrm{C}^{*}$-algebra associated with an $X$-module.

1.2.8 Definition (Roe $\mathrm{C}^{*}$-algebra of an $X$-module). Let $(\mathscr{H}, \rho)$ be an $X$ module. The Roe $\mathrm{C}^{*}$-algebra or coarse $\mathrm{C}^{*}$-algebra of $(\mathscr{H}, \rho)$ is the $\mathrm{C}^{*}$ algebra generated by all operators $T \in \mathscr{L}_{A}^{*}(\mathscr{H})$ which are locally compact and of finite propagation:

$$
\mathrm{C}^{*}(\mathscr{H}, \rho):=\mathrm{C}^{*}\{\text { locally compact, finite propagation ops. }\} \text {. }
$$

Thus $\mathrm{C}^{*}(\mathscr{H}, \rho)=\mathrm{C}^{*}\left(\Psi_{\mathrm{fp}}^{-1}(X)\right)$.

Next we introduce a relative version of the Roe $\mathrm{C}^{*}$-algebra. This will take a preeminent role in the proof of the vanishing theorem in Section 1.4.6.

1.2.9 Definition (Relative Roe $\mathrm{C}^{*}$-algebra). Let $(\mathscr{H}, \rho)$ be an $X$-module and $K \subset X$ a subset. An operator $T \in \mathscr{L}_{A}^{*}(\mathscr{H})$ with finite propagation is supported near $K$ if there exists $R>0$ such that

$$
\rho(\varphi) T=T \rho(\varphi)=0
$$

for each $\varphi \in C_{0}(X)$ with $d(\operatorname{supp}(\varphi), K)>R$. The Roe $\mathrm{C}^{*}$-algebra or coarse $\mathrm{C}^{*}$-algebra of $(\mathscr{H}, \rho)$ relative to $K$ is the $\mathrm{C}^{*}$-algebra generated by all $T \in$ $\Psi_{\mathrm{fp}}^{-1}(X)$ which are supported near $K$ and is denoted by $\mathrm{C}_{K}^{*}(\mathscr{H}, \rho)$.

The following lemma gives another description of the relative Roe $\mathrm{C}^{*}$-algebra. 


\section{Coarse index theory and positive scalar curvature}

1.2.10 Lemma. Let $X$ be a locally compact metric space, $(\mathscr{H}, \rho)$ an admissible $X$-module and $K \subset X$ a compact subset. Assume $\rho$ extends to a unital $\mathrm{C}^{*}$ homomorphism on $C(X)$. The following holds:

$$
\mathrm{C}_{K}^{*}(\mathscr{H}, \rho)=\mathrm{C}^{*}\left\{T \in \Psi_{\mathrm{fp}}^{-1}(X) ; T \text { has property }(*)\right\}
$$

where the property (*) reads as follows: for each $\varepsilon>0$ there exists $R>0$ such that $\|T u\|<\varepsilon$ for all compactly supported $u \in \mathscr{H}$ with $\|u\|=1$ and $d(\operatorname{supp}(u), K)>R$.

Proof. "C": Let $T \in \Psi_{\mathrm{fp}}^{-1}(X) \subset \mathscr{L}_{A}^{*}(\mathscr{H})$ be supported near $K$ and $\varepsilon>0$. Then there exists $R>0$ such that $T \rho(\varphi)=\rho(\varphi) T=0$ for each $\varphi \in C_{0}(X)$ with $d(\operatorname{supp}(\varphi), K)>R$. Assume $u \in \mathscr{H}$ is compactly supported and $d(\operatorname{supp}(u), K)>R+1$. Since $X$ is locally compact, there exists by [30, Section 2.4] a function $\psi \in C_{\mathrm{cpt}}(X)$ which is equal to 1 on a small neighbourhood of $\operatorname{supp}(u)$ and whose support satisfies $d(\operatorname{supp}(\psi), K)>R$. But this implies $\|T u\|=\|T \rho(\psi) u\|=0<\varepsilon$ showing that $T$ satisfies $(*)$.

" $\supset$ ": Let $T \in \Psi_{\mathrm{fp}}^{-1}(X)$ be a locally compact operator of finite propagation which satisfies property $(*)$. Let $\varepsilon>0$. We show that there exists an operator $T_{\varepsilon} \in \Psi_{\mathrm{fp}}^{-1}(X)$ which is supported near $K$ and which satisfies $\left\|T-T_{\varepsilon}\right\| \leq \varepsilon$. This implies $T \in \mathrm{C}_{K}^{*}(\mathscr{H}, \rho)$.

Given $\varepsilon>0$, there exists $R>0$ such that $\|T u\|<\varepsilon$ for all compactly supported $u \in \mathscr{H}$ with $\|u\|=1$ and $\operatorname{supp}(u) \cap \overline{B(K ; R)} \neq \varnothing$. By [30, Section 2.4] there exists a function $\varphi_{\varepsilon} \in C_{\mathrm{cpt}}(X)$ with $\overline{B(K ; R+1)} \subset \operatorname{supp}\left(\varphi_{\varepsilon}\right)$ with $\varphi_{\mid \overline{B(K ; R+1)}}=$ 1. Set $T_{\varepsilon}:=T \rho\left(\varphi_{\varepsilon}\right)$. Then $T_{\varepsilon}$ is locally compact and has finite propagation since $T$ is already of this kind. Furthermore, $(*)$ implies

$$
\left\|T u-T_{\varepsilon} u\right\|=\left\|T \rho\left(1-\varphi_{\varepsilon}\right) u\right\| \leq \varepsilon .
$$

From this $\left\|T-T_{\varepsilon}\right\| \leq \varepsilon$ follows because $\mathscr{H}_{\text {cpt }}$ is dense in $\mathscr{H}$.

1.2.11 Notation. From now on $\mathscr{H}$ will be as in Example 1.2.2. In this case we write $\mathrm{C}^{*}(M ; A)$ for $\mathrm{C}^{*}\left(L^{2}(M, E), \rho\right)$ or $\mathrm{C}^{*}(M, \mathscr{H})$ if we want to make the module $\mathscr{H}$ explicit. Analogously, $\mathrm{C}_{K}^{*}(M ; A)$ stands for $\mathrm{C}_{K}^{*}\left(L^{2}(M, E), \rho\right)$.

Finally, we prove the following important lemma about the relative Roe $\mathrm{C}^{*}$ algebra of a compact subset.

1.2.12 Lemma. If $K$ is compact, then $\mathrm{C}_{K}^{*}(M ; A)$ equals $\mathscr{K}_{A}\left(L^{2}(M, E)\right)$.

Proof. "つ": Let $\left(e_{j}\right)_{j \in \mathbb{N}}$ be an orthonormal basis of $L^{2}(M, E)$ consisting of compactly supported elements ${ }^{4}$. We claim that each elementary compact operator ${ }^{5}$ $\Theta_{e_{j} a, e_{k} b}$ for $j, k \in \mathbb{N}$ and $a, b \in A$ is supported near $K$ and locally compact. After proving this the claim follows since $\mathscr{K}_{A}\left(L^{2}(M, E)\right)$ is the $\mathrm{C}^{*}$-algebra generated by all elementary compact operators, and since $x_{n} \rightarrow x$ and. $y_{n} \rightarrow y$

\footnotetext{
${ }^{4}$ One can use Claim 6.3.14 from [21] to obtain such a basis for each ample non-degenerate module.

${ }^{5}$ See Definition 1.4.10 for our notation.
} 
implies $\Theta_{x_{n}, y} \rightarrow \Theta_{x, y}$ and $\Theta_{x, y_{n}} \rightarrow \Theta_{x, y}$. So let $j, k \in \mathbb{N}$ and $a, b \in A$. Since each compact operator is locally compact, we only have to prove that $\Theta_{e_{j} a, e_{k} b}$ is supported near $K$.

To see that $\Theta_{e_{j} a, e_{k} b}$ has finite propagation, choose $R>0$ such that $\operatorname{supp}\left(e_{j}\right) \subset$ $B\left(\operatorname{supp}\left(e_{k}\right) ; R\right)$. Then $\operatorname{supp}\left(\Theta_{e_{j} a, e_{k} b} u\right)$ is contained in the ball around $\operatorname{supp}(u)$ of radius $R+\operatorname{diam}\left(\operatorname{supp}\left(e_{k}\right)\right)$. In order to see that $\Theta_{e_{j} a, e_{k} b}$ is supported near $K$, choose $R>0$ such that

$$
\operatorname{supp}\left(e_{j}\right) \cup \operatorname{supp}\left(e_{k}\right) \subset \overline{B(K ; R)} .
$$

Then one has for any $u \in L^{2}(M, E)$

$$
\left(\rho(\varphi) \Theta_{e_{j} a, e_{k} b}\right) u=\left(\rho(\varphi) e_{j} a\right)\left\langle e_{k} b, u\right\rangle=0
$$

and

$$
\Theta_{e_{j} a, e_{k} b}(\rho(\varphi) u)=\left(e_{j} a\right)\left\langle e_{k} b, \rho(\varphi) u\right\rangle=\left(e_{j} a\right)\left(\int_{M}\left(e_{k}(x) b\right) \varphi(x) u(x) d x\right)=0
$$

for any $\varphi \in C_{0}(X)$ with $\operatorname{supp}(\varphi) \cap \overline{B(K ; R)}=\varnothing$.

"C": Let $T \in \Psi_{\mathrm{fp}}^{-1}(X)$ be supported near $K$ and choose $R>0$ as in Definition 1.2.9. Since $K$ is compact, there exists a covering $\left(U_{j}\right)_{j \in \mathbb{N}}$ of $M$ by open subsets such that only finitely many of these have non-empty intersection with $\overline{B(K ; R)}$. Let $\left(\pi_{j}\right)_{j \in \mathbb{N}}$ be a partition of unity subordinated to $\left(U_{j}\right)_{j \in \mathbb{N}}$. We choose the notation in such a way that $\left\{j \in \mathbb{N} ; \operatorname{supp}\left(\pi_{j}\right) \cap \overline{B(K ; R)} \neq \varnothing\right\}$ is $\{1, \ldots, n\}$. If $N \geq n+1$ and $u \in L^{2}(M, E)$, then one has

$$
\left\|T u-\sum_{j=1}^{n} \rho\left(\pi_{j}\right) T u\right\|=\left\|\rho\left(f_{N}\right) T u\right\|
$$

with $f_{N}:=1-\sum_{j=1}^{N} \pi_{j}$, since $d\left(\operatorname{supp}\left(\pi_{j}\right), K\right)>R$ for $j \geq n+1$. The claim follows now because $\rho\left(f_{N}\right) \rightarrow 0$ (strongly). To see this let $v \in \Gamma_{\mathrm{cpt}}(M, E)$. Then

$$
\left\|\rho\left(f_{N}\right) v\right\|^{2}=\int_{M}\left|f_{N}(x)\right|^{2}\|v(x)\|^{2} d x
$$

becomes zero for sufficiently large $N$ as $f_{N}(x)=0$ on $\operatorname{supp}(v)$ for such $N$. The desired result follows now because $\Gamma_{\mathrm{cpt}}(M, E)$ is dense in $L^{2}(M, E)$.

\subsection{Coarse indices}

Let $(M, g)$ be a complete Riemannian spin manifold with Dirac operator $D$. In this section we define the even and odd coarse index (class)

$$
\operatorname{ind}_{p}(D) \in \mathrm{K}_{p}\left(\mathrm{C}^{*}(M, A)\right) \quad, \quad p=0,1 .
$$

For this we will use the functional calculus for so-called regular operators on Hilbert $\mathrm{C}^{*}$-modules. We describe this calculus in the next subsection. 


\subsubsection{The functional calculus for regular operators}

The aim of this section is to introduce the functional calculus which in the end will allow us to define the operator $\chi(D)$ (where $\chi$ is a normalizing function) used in the definition of the coarse index. Before this, the calculus will be used to introduce the wave semi-group $\left\{e^{i s D}\right\}_{s \in \mathbb{R}}$. This is a one-parameter family of unitary operators by means of which we can express the functions $\varphi(D)$ of $D$ (where $\varphi$ is a function in $C_{0}(\mathbb{R})$ ) via Fourier transformation as $(2 \pi)^{-1} \int \hat{\varphi}(t) e^{i t D} d t$. This will enable us to show that $\varphi(D)$ belongs to the Roe $\mathrm{C}^{*}$-algebra for $\varphi \in C_{0}(\mathbb{R})$.

The notion of a regular operator on a Hilbert module is due to Woronowicz and Baaj-Julg. Our presentation if based mainly on [28] and [50].

1.3.1 Definition (Regular operator). A closed $^{6} A$-linear operator $T: \mathfrak{d o m}(T) \rightarrow \mathscr{H}$ with domain $\mathfrak{d o m}(T) \subset \mathscr{H}$ is called regular if

(i) Both $T$ and $T^{*}$ are densely defined.

(ii) The range of $1+T^{*} T$ is dense.

We denote the set of all regular operators on the Hilbert $A$-module $\mathscr{H}$ by $\mathscr{R}_{A}(\mathscr{H})$.

The following theorem guarantees that the Dirac-type operator we are mostly interested in is regular in the sense of Definition 1.3.1.

1.3.2 Theorem. Let $(M, g)$ be a complete Riemannian spin manifold with spinor bundle $\Sigma M$. The closure

$$
D: H^{1}(M, S) \rightarrow L^{2}(M, S)
$$

of the twisted spinoral Dirac operator, with twisting bundle the Mishchenko line bundle,

$$
D: \Gamma_{\mathrm{cpt}}(M, S) \rightarrow \Gamma_{\mathrm{cpt}}(M, S) \subset L^{2}(M, S)
$$

with $S=\Sigma M \otimes \mathscr{V}_{M}$, is regular and self-adjoint.

Proof. See [51, Lemma 2.1].

The next theorem introduces the continuous functional calculus for normal regular operators.

Recall that the spectrum $\sigma(T)$ of an (unbounded) operator $T$ on a Hilbert $\mathrm{C}^{*}$-module $\mathscr{H}$ over a $\mathrm{C}^{*}$-algebra $A$ is simply the spectrum of $T$ viewed as an (unbounded) operator on the particular Banach space $\mathscr{H}$. Thus $\sigma(T)$ is the complement of the resolvent set $\rho(T) \subset \mathbb{C}$, where $\lambda \in \rho(T)$ if and only if the operator $\rho(\lambda):=(T-\lambda I): \mathfrak{d o m}(D) \rightarrow \mathscr{H}$ has a bounded inverse. Notice that $\rho(\lambda)$ is automatically $A$-linear but it is not clear whether $\rho(\lambda) \in \mathscr{L}_{A}^{*}(\mathfrak{d o m}(T), \mathscr{H})$ or not. In particular, $0 \in \sigma(T)$ if and only if $T: \mathfrak{d o m}(T) \rightarrow \mathscr{H}$ admits no bounded inverse.

\footnotetext{
${ }^{6}$ This is defined exactly as in the Hilbert space case (i.e. $A=\mathbb{C}$ ).
} 
1. Coarse index theory and positive scalar curvature

1.3.3 Theorem (Continuous functional calculus). Let $T \in \mathscr{R}_{A}(\mathscr{H})$ be a normal regular A-linear operator on the Hilbert A-module $\mathscr{H}$. Then there exists a map

$$
\pi_{T}: C(\mathbb{C}) \rightarrow \mathscr{R}_{A}(\mathscr{H}), f \mapsto f(T)
$$

with the following properties:

(i) $\left(\operatorname{id}_{\mathbb{C}}\right)(T)=T$ and $\pi_{T}(1)=I$.

(ii) $(f+\lambda g)(T)=f(T)+\lambda g(T)$ for any $\lambda \in \mathbb{C}$.

(iii) $(f \cdot g)(T)=\overline{f(T) \circ g(T)}=\overline{g(T) \circ f(T)}$.

(iv) If $f \in B C(\mathbb{C})$ is a bounded continuous function, then $f(T)$ is bounded.

(v) If $f, g \in C(\mathbb{C})$ coincide on $\sigma(T)$, then $f(T)=g(T)$.

(vi) Spectral mapping theorem: One has $\sigma(f(T))=\overline{f(\sigma(T))}$ for $f \in C_{0}(\mathbb{C})$.

(vii) If $f \in B C(\mathbb{C})$, then $\|f(T)\|=\|f\|_{\sigma(T)}$ is the supremum of $|f|$ on $\sigma(T)$.

(viii) If $g \in B C(\mathbb{C})$, then $(f \circ g)(T)=f(g(T))$ for any $f \in C(\mathbb{C})$.

(ix) $f(T)^{*}=f\left(T^{*}\right)$.

(x) If $T$ is bounded, then $\pi_{T}$ is the usual functional calculus of the normal element $T$ in the $C^{*}$-algebra $\mathscr{L}_{A}^{*}(\mathscr{H})$.

(xi) Continuity property: Assume $\left(f_{n}\right)_{n \in \mathbb{N}} \subset C(\mathbb{C})$ is a sequence of continuous functions and $F \in C(\mathbb{C})$ is such that $\left|f_{n}\right| \leq|F|$. If $f_{n} \rightarrow f$ uniformly on compact subsets, then $f_{n}(T) \rightarrow f(T)$ pointwise on $\mathfrak{d o m}(F(T))$.

(xii) The restriction $\pi_{T}: C_{0}(\mathbb{C}) \rightarrow \mathscr{L}_{A}^{*}(E)$ is a $\mathrm{C}^{*}$-algebra homomorphism.

Proof. We only need to prove (v), (vi) and (vii) here since a proof of the remaining statements can be found in [28, p. 473 ff., especially Prop. 16 on p. 474]. Before doing so we briefly outline the construction of the functional calculus from [28].

Let $\mathbb{D} \subset \mathbb{C}$ denote the open unit disk. The map $h: \mathbb{C} \rightarrow \mathbb{D}, h(z)=z(1+$ $\left.|z|^{2}\right)^{-1 / 2}$ is a bijection and using it we define a map $-: C_{0}(\mathbb{C}) \rightarrow C_{0}(\mathbb{D})$ by mapping $g \in C_{0}(\mathbb{C})$ to the function $\bar{g} \in C_{0}(\mathbb{D})$ with $\bar{g}(h(z)):=h(g(z))$. Next let $Q: \mathscr{R}_{A}(\mathscr{H}) \rightarrow \mathscr{V}_{A}(\mathscr{H})$ with $Q(T):=T\left(1+T^{*} T\right)^{-1 / 2}$ be the bounded transform from [28, Prop. 10] and $\Phi: C(\mathbb{C}) \rightarrow \mathscr{L}_{A}^{*}(\mathscr{H})$ with $\Phi(f)=f(Q(T))$ the functional calculus of the now bounded operator $Q(T)$. Because $Q$ maps $\mathscr{L}_{A}^{*}(\mathscr{H}) \subset \mathscr{R}_{A}(\mathscr{H})$ onto the open unit disc in $\mathscr{L}_{A}^{*}(\mathscr{H})$, see again [28, Prop. 10], and therefore if $\|f\|_{\infty}<1$ whence also $\|f(Q(T))\|<1$, there exists $T_{f} \in$ $\mathscr{L}_{A}^{*}(\mathscr{H})$ with $Q\left(T_{f}\right)=f(Q(T))$ or $T_{f}=Q^{-1}(f(Q(T)))$. Using this the desired functional calculus $\pi_{T}: C_{0}(\mathbb{C}) \rightarrow \mathscr{L}_{A}^{*}(\mathscr{H})$ is finally defined by $\pi_{T}(g):=T_{\bar{g}}$.

This calculus for functions in $C_{0}(\mathbb{C})$ can be extended to a calculus for arbitrary continuous functions as follows: by [28, Cor. 14] the algebraic tensor product $C_{0}(\mathbb{C}) \odot_{\pi_{T}} \mathscr{H}$ is isomorphic to $\mathscr{H}$ via the map that sends the elementary tensors 


\section{Coarse index theory and positive scalar curvature}

$f \odot e$ onto $f(T) e$. Using this the given action $\pi_{T}: C_{0}(\mathbb{C}) \rightarrow \mathscr{L}_{A}^{*}(\mathscr{H})$ induces an action $\pi_{T}: C(\mathbb{C}) \rightarrow \mathscr{R}_{A}(\mathscr{H})$ as follows: let $\pi_{T}(f)$ for $f \in C(\mathbb{C})$ be the operator whose domain $\mathfrak{d o m}\left(\pi_{T}(f)\right)$ is the linear span generated by those elementary tensors $h \odot e$ (with $e \in \mathscr{H}$ and $h \in C_{0}(\mathbb{C})$ ) for which $f h \in C_{0}(\mathbb{C})$ and which maps $h \odot e$ to $(f h) \odot e$. With this definition $f(T)$ is clearly defined everywhere whenever $f$ is bounded.

(v) We can assume $\rho(T) \neq \varnothing$. By linearity, it suffices to show that $f(T)=0$ if $f$ vanishes on $\sigma(T)$. First of all, assume that $f$ is compactly supported with $\operatorname{supp}(f) \subset B\left(\lambda ; \varepsilon_{\lambda}\right)$ where $\lambda \in \rho(T)$ and $\varepsilon_{\lambda}>0$ is such that $\left\|(T-\lambda)^{-1}\right\| \leq \varepsilon_{\lambda}^{-1}$. Then we can write

$$
\begin{aligned}
f(T) & =f(T) \circ(T-\lambda)^{n} \varepsilon_{\lambda}^{-n} \circ(T-\lambda)^{-n} \varepsilon_{\lambda}^{n} \\
& =\overline{f(T) \circ(T-\lambda)^{n} \varepsilon_{\lambda}^{-n}} \circ(T-\lambda)^{-n} \varepsilon_{\lambda}^{n} \\
& =f_{n}(T) \circ(T-\lambda)^{-n} \varepsilon_{\lambda}^{n}
\end{aligned}
$$

by (iii), where $f_{n}(t):=f(t)(t-\lambda)^{n} \varepsilon_{\lambda}^{-n}$. Here we have used that $f(T)$ is continuous by (iv) and that $T$ is closed. Thus

$$
\|f(T)\| \leq\left\|f_{n}(T)\right\|\left\|(T-\lambda)^{-n} \varepsilon_{\lambda}^{n}\right\| \leq\left\|f_{n}\right\|_{\infty}
$$

since $\pi_{T}$ is continuous with $\left\|\pi_{T}\right\|=1$. Since $\operatorname{supp}(f)$ lies in $B\left(\lambda ; \varepsilon_{\lambda}\right)$ and is compact, there exists $0<q<1$ with $|t-\lambda| \varepsilon_{\lambda}^{-1}<q$ for all $t \in \operatorname{supp}(f)$, implying $\|f(T)\|=0$. Secondly, assume $f$ compactly supported in $\rho(T)$. There exist $\lambda_{1}, \ldots, \lambda_{n} \in \rho(T)$ and $\varepsilon_{1}, \ldots, \varepsilon_{n}>0$ such that the balls $B\left(\lambda_{j} ; \varepsilon_{j}\right)$ cover $\operatorname{supp}(f)$ and such that $\left\|T-\lambda_{j}\right\| \leq \varepsilon_{j}^{-1}$. Let $\left\{\pi_{1}, \ldots, \pi_{n}\right\}$ be a partition of unity subordinated to open covering given by these balls. Then $\left(f \cdot \pi_{j}\right)(T)=0$ by the previous part and thus also $f(T)=0$ in this case. Finally, we remark that each $f$ which vanishes on $\sigma(T)$ is a uniform limit of functions compactely supported in $\rho(T)$ whose absolute values are bounded by $|f|$, so that (ix) implies also $f(T)=0$ in this case.

(vi) The claim follows once we have shown a corresponding version of it holds for $q$ : indeed the spectral mapping theorem for the normal operator $Q(T)$ together with $\sigma(q(T))=\overline{q(\sigma(T))}$ implies

$$
\begin{aligned}
\sigma(f(T)) & =\sigma\left(\pi_{T}(f)\right) \\
& =\sigma\left(\left(f \circ q^{-1}\right)(Q(T))\right) \\
& =\left(f \circ q^{-1}\right)(\sigma(Q(T))) \\
& =\left(f \circ q^{-1}\right)(\sigma(q(T))) \\
& =\left(f \circ q^{-1}\right)(\overline{q(\sigma(T))}) \\
& =\overline{f(\sigma(T))}
\end{aligned}
$$

for each $f \in C_{0}(\mathbb{C})$. Here the inclusion " $\subset$ " for the last equality is obvious. In order to show the other inclusions " $\supset$ " it suffices to show $\left(f \circ q^{-1}\right)(\overline{q(\sigma(T))}) \supset$ $f(\sigma(T))$ (as the LHS in the inclusion is closed by the above). The latter then easily follows from $\overline{q(\sigma(T))} \supset q(\sigma(T))$. 
Furthermore, one can prove $\sigma(f(T)) \subset \overline{f(\sigma(T))}$ for any $f \in B C(\mathbb{C})$ directly in the following way: let $\mu \notin \overline{f(\sigma(T))}$ and let $g_{\mu}: \mathbb{C} \rightarrow \mathbb{C}$ be a bounded continuous extension of the bounded continuous function on $\sigma(T)$ whose value in $t \in \sigma(T)$ is $(f(t)-\mu)^{-1}$. By (iii) and (v)

$$
\begin{aligned}
g_{\mu}(T) \circ(f(T)-\mu I) & =\overline{g_{\mu}(T) \circ(f(T)-\mu I)} \\
& =\left(g_{\mu} \cdot\left(f-\mu \operatorname{id}_{\mathbb{C}}\right)\right)(T) \\
& =\pi_{T}(1) \\
& =T
\end{aligned}
$$

and likewise

$$
\begin{aligned}
(f(T)-\mu I) \circ g_{\mu}(T) & =\overline{(f(T)-\mu I) \circ g_{\mu}(T)} \\
& =\left(\left(f-\mu \mathrm{id}_{\mathbb{C}}\right) \cdot g_{\mu}\right)(T) \\
& =\pi_{T}(1) \\
& =T
\end{aligned}
$$

Thus $\mu \notin \sigma(f(T))$. Here we have used that $g_{\mu}(T)$ and $f(T)$ are bounded. Therefore $\sigma(f(T)) \subset \overline{f \sigma(T))}$ and in particular $\sigma(q(T)) \subset \overline{q(\sigma(T))}$.

We are left to prove $\overline{q(\sigma(T))} \subset \sigma(q(T))$. For this suppose there exists $\lambda \in \sigma(T)$ such that $q(\lambda) \notin \sigma(q(T))$. Then there exists a bounded operator $G$ which inverts $(q(T)-q(\lambda) I)$. Furthermore, there exists $g_{\lambda} \in B C(\mathbb{C})$ with $(q(T)-q(\lambda) I)=$ $(T-\lambda I) g_{\lambda}(T)$. But this implies that the bounded operator $G \circ g_{\lambda}(T)$ inverts $(T-\lambda I)$ contradicting $\lambda \in \sigma(T)$. Thus $q(\sigma(T)) \subset \sigma(q(T))$ and as a consequence thereof $\overline{q(\sigma(T))} \subset \sigma(q(T))$. To see that $G \circ g_{\lambda}(T)$ would really invert $(T-\lambda I)$ we notice that both

$$
\begin{aligned}
I & =G \circ(q(T)-q(\lambda) I) \\
& =G \circ \overline{(T-\lambda I) \circ g_{\lambda}(T)} \\
& =G \circ \overline{(T-\lambda I) \circ g_{\lambda}(T)} \\
& =G \circ \overline{g_{\lambda}(T) \circ(T-\lambda I)} \\
& =G \circ g_{\lambda}(T) \circ(T-\lambda I)
\end{aligned}
$$

and analogously

$$
\begin{aligned}
I & =(q(T)-q(\lambda) I) \circ G \\
& =(T-\lambda I) \circ g_{\lambda}(T) \circ G
\end{aligned}
$$

hold by (iii). Here we have used that $T$ is closed and $g_{\lambda}(T)$ is bounded to see that $(T-\lambda I) \circ q_{\lambda}(T)$ is bounded, as well as that $g_{\lambda}(T)$ and $G$ are both functions of $Q(T)$ and hence commute.

(vii) We have already seen $\|f(T)\| \leq\|f\|_{\infty}$ in the proof of part (v). But by (v) we can replace $\|f\|_{\infty}$ here by $\|f\|_{\sigma(T)}$. This can be seen the following way: since $f$ is bounded, $\sigma(f(T))$ is compact. Furthermore we know $\|f(T)\|=$ $\|g(T)\|=\|g\|_{\infty}$ by $(\mathrm{v})$ for any $g \in B C(\mathbb{C})$ which coincides with $f$ on $\sigma(T)$. If we assume $\|f(T)\|>\|f\|_{\sigma(T)}$ this yields a contradiction. Simply let $\phi$ be a Urysohn function for the pair $(\sigma(T), \mathbb{C} \backslash U)$, where $U$ is an open neighbourhood 
of $\sigma(T)$, and set $g:=\phi \cdot f$. On the other hand the spectral radius formula together with (vi) implies

$$
\begin{aligned}
\|f(T)\| & \geq \sup \{|z| ; z \in \sigma(f(T))\} \\
& =\sup \{|w| ; w \in \overline{f(\sigma(T))}\} \\
& \geq \sup \{|w| ; w \in f(\sigma(T))\} \\
& =\|f\|_{\sigma(T)}
\end{aligned}
$$

and thus the desired relation.

The following lemma is used in the proof of Theorem 1.3.5.

1.3.4 Lemma. Let $W$ be the set of all functions $\varphi \in C_{0}(\mathbb{R}) \cap L^{1}(\mathbb{R})$ such that $\operatorname{supp}(\hat{\varphi})$ is compact ${ }^{7}$. Then there exists for each $\varphi \in C_{0}(\mathbb{R})$ a sequence $\left(\varphi_{n}\right)_{n \in \mathbb{N}}$ in $W$ with $\varphi_{n} \rightarrow \varphi$ uniformly and $\left|\varphi_{n}\right| \leq|\Phi|$ for a constant function $\Phi \in C(\mathbb{R})$. In particular, $W$ is dense in $C_{0}(\mathbb{R})$, and $\varphi_{n}(D)$ converges strongly to $\varphi(D)$ on all of $\mathscr{H}$ according to part (viii) of Theorem 1.3.3

Proof. Let $\psi \in C_{0}(\mathbb{R})$. We will show that to each $n \in \mathbb{N}$ there exists a $\varphi_{n} \in W$ such that

$$
\sup _{t \in \mathbb{R}}\left|\psi(t)-\varphi_{n}(t)\right|<\frac{1}{n}
$$

To achieve this let $n \in \mathbb{N}$ and choose first of all a Schwartz function $f_{n} \in \mathscr{S}(\mathbb{R})$ with

$$
\sup _{t \in \mathbb{R}}\left|\psi(t)-f_{n}(t)\right|<\frac{1}{2 n} .
$$

Since $C_{\mathrm{cpt}}^{\infty}(\mathbb{R})$ is dense in $L^{1}(\mathbb{R})$ and because of the Fourier inversion formula we can find $\varphi_{n} \in W$ given by $\varphi_{n}(t)=\int \hat{\varphi}_{n}(s) e^{i s t} d t$ such that

$$
\sup _{t \in \mathbb{R}}\left|f_{n}(t)-\varphi_{n}(t)\right| \leq\left\|\hat{f}_{n}-\hat{\varphi}_{n}\right\|_{1}<\frac{1}{2 n} \text {. }
$$

Thus

$$
\sup _{t \in \mathbb{R}}\left|\psi(t)-\varphi_{n}(t)\right|<\frac{1}{n}
$$

for this choice of $\varphi_{n}$. Furthermore, $\left\|\varphi_{n}\right\|_{\infty}$ converges to $\|\psi\|_{\infty}$, which allows to obtain a function $\Phi \in C(\mathbb{R})$ as desired.

Let $D \in \mathscr{R}_{\mathrm{C}^{*} \pi}\left(L^{2}(M, S)\right)$ be as in Theorem 1.3.2. (Most of the following results could be stated in more general form for an arbitrary self-adjoint operator $T \in \mathscr{R}_{A}(\mathscr{H})$ instead of $D$.) One has $\sigma(D) \subset \mathbb{R}$ and can thus consider the family $\{U(s)\}_{s \in \mathbb{R}}$ given by the operators

$$
U(s):=\exp (i s D)
$$

for $s \in \mathbb{R}$, which are obtained from the operator $D$ by applying the functional calculus $\pi_{D}$ to the bounded function $f: \mathbb{R} \rightarrow \mathbb{C}$ with $f(t):=\exp (i s t)$.

The following theorem is the main result of this section.

\footnotetext{
${ }^{7}$ Here we use the notation used in [37, p. 45]. The set $W$ contains, e.g., the sinus cardinalis, which is given by $\operatorname{sinc}(t)=t^{-1} \sin (t)$ for $t \in \mathbb{R}$.
} 
1. Coarse index theory and positive scalar curvature

1.3.5 Theorem. The following hold:

(i) The family $\{U(s)\}_{s \in \mathbb{R}}$ is a one-parameter group of (bounded) unitary Alinear operators, i.e. the map $U: \mathbb{R} \rightarrow \mathscr{U}_{A}(\mathscr{H}), s \mapsto U(s)$ is a group homomorphism.

(ii) The one-parameter group $\{U(s)\}_{s \in \mathbb{R}}$ solves the wave equation ${ }^{8} U^{\prime}(s)=$ $i D U(s)$ uniquely for the initial condition

$$
\lim _{s \rightarrow 0} U(s) u=u \quad, \quad u \in \mathfrak{d o m}(D)
$$

and is called the wave semigroup.

(iii) Each operator $U(s)$ is an operator of finite propagation in the sense of Definition 1.2.5.

(iv) One can express the functional calculus of $D$ with $\{U(s)\}_{s \in \mathbb{R}}$ via Fourier transformation as

$$
\varphi(D)=\frac{1}{2 \pi} \int_{\mathbb{R}} \hat{\varphi}(s) U(s) d s
$$

for $\varphi \in W$, where $W$ is the set of all (bounded) smooth functions $\varphi \in C^{\infty}(\mathbb{R}) \cap L^{1}(\mathbb{R})$ such that $\hat{\varphi} \in C_{\mathrm{cpt}}^{\infty}(\mathbb{R})$ is compactly supported. Here Equation (1.3.8) holds in the weak sense, i.e. one has

$$
\langle\varphi(D) u, v\rangle=\frac{1}{2 \pi} \int_{\mathbb{R}} \hat{\varphi}(s)\langle U(s) u, v\rangle d s
$$

for $u, v \in \Gamma_{\mathrm{cpt}}(M, S)$ and the right-hand side is the Riemann integral of a bounded continuous function. Cf. [21, Prop. 10.3.5].

(v) As a consequence of (iii) and (iv), one has $\varphi(D) \in \mathrm{C}^{*}(\mathscr{H}, \rho)$ for $\varphi \in$ $C_{0}(\mathbb{R})$.

Proof. (i) It follows from part (iv) of Theorem 1.3.3 that each operator $U(s)$ is bounded. That these operators are unitary, follows from (vi) of Theorem 1.3.3 since $D$ is self-adjoint by Theorem 1.3.2: we have

$$
U(s)^{*}=\exp \left(-i s D^{*}\right)=\exp (-i s D)=U(-s) .
$$

Analogously, we obtain from (iii) of Theorem 1.3.3 that

$$
U\left(s+s^{\prime}\right)=\exp \left(i\left(s+s^{\prime}\right) D\right)=\exp (i s D) \circ \exp \left(i s^{\prime} D\right)=U(s) \circ U\left(s^{\prime}\right) .
$$

Thus $U: \mathbb{R} \rightarrow \mathscr{U}_{A}(\mathscr{H})$ is a group homomorphism.

(ii) We show that for each $s_{0} \in \mathbb{R}$ the limit

$$
U^{\prime}\left(s_{0}\right)=\lim _{s \rightarrow s_{0}} \frac{U(s)-U\left(s_{0}\right)}{s-s_{0}}
$$

\footnotetext{
${ }^{8}$ Here the limit $U^{\prime}(s)$ exists pointwise and equals $i D U(s)$.
} 


\section{Coarse index theory and positive scalar curvature}

exists pointwise and equals $i D U\left(s_{0}\right)$. For this let $\left(s_{n}\right)_{n \in \mathbb{N}}$ be any sequence converging to $s_{0}$ and $C>0$ such that $\left|s_{n}-s_{0}\right|<C$ for each $n \in \mathbb{N}$. We have

$$
\frac{U\left(s_{n}\right)-U\left(s_{0}\right)}{s_{n}-s_{0}}=f_{n}(D)
$$

with $f_{n}(t)=\left(\exp \left(i s_{n} t\right)-\exp \left(i s_{0} t\right)\right)\left(s_{n}-s_{0}\right)^{-1}$. Obviously, $f_{n} \rightarrow f$ pointwise for $f(t):=i t \exp \left(i s_{0} t\right)$. As we show next, the convergence $f_{n} \rightarrow f$ is even uniform on compact subsets. For this end, assume $|t| \leq B$. A straightforward computation then shows

$$
\left|f_{n}(t)-f(t)\right| \leq\left(\sum_{j=2}^{\infty} \frac{C^{j-2} B^{j}}{j !}\right)\left|s_{n}-s_{0}\right|
$$

which proves that $f_{n} \rightarrow f$ uniformly on $[-B, B]$. An easy computation shows

$$
\begin{aligned}
\left|f_{n}(t)\right| & =\left|\frac{\exp \left(i s_{n} t\right)-\exp \left(i s_{0} t\right)}{s_{n}-s_{0}}\right| \\
& =\left|\frac{1}{s_{n}-s_{0}}\left(\int_{s_{0} t}^{s_{n} t} e^{i \tau} d \tau\right)\right| \\
& \leq|t| .
\end{aligned}
$$

Since the sequence of functions $\left(f_{n}\right)_{n \in \mathbb{N}}$ also converges uniformly on compact subset, we can now conclude from part (viii) of Theorem 1.3.3 that $f_{n}(D) \rightarrow$ $f(D)$ pointwise on $\mathfrak{d o m}(D)$.

In order to show that the wave equation is uniquely solvable for the given initial condition, notice that the self-adjointness of $D$ implies

$$
\frac{d}{d s}\|C(s) u\|^{2}=0
$$

for any $u \in \mathfrak{d o m}(D)$, whenever $\{C(s)\}_{s \in \mathbb{R}}$ is a solution of the wave equation.

(iii) Cf. [21, Prop. 10.3.1] and its proof.

(iv) Let $u, v \in \Gamma_{\text {cpt }}(M, S)$. Suppose $\operatorname{supp}(\hat{\varphi}) \subset[-R, R]$ for $R>0$. As the following remarks show, the function $s \mapsto\langle U(s) u, v\rangle$ of $s \in[-R, R]$ is continuous. If $\left(s_{n}\right)_{n \in \mathbb{N}}$ is a sequence in $[-R, R]$ converging to $s_{0}$, then we can first of all estimate as follows:

$$
\begin{aligned}
\left|\left\langle\left(U\left(s_{n}\right)-U\left(s_{0}\right)\right) u, v\right\rangle\right| & \leq\left\|\left(U\left(s_{n}\right)-U\left(s_{0}\right)\right) u\right\|\|v\| \\
& \leq\left\|U\left(s_{0}\right)\right\|\left\|\left(U\left(s_{n}-s_{0}\right)-I\right) u\right\|\|v\| .
\end{aligned}
$$

Set $h_{n}:=s_{n}-s_{0}$. The operator $U\left(h_{n}\right)-I$ is the operator obtained from $D$ by applying the function $f_{n}(t):=\exp \left(i h_{n} t\right)-1$ to it. Notice that (writing $f_{h}(t)$ for $\exp (i h t)-1)$

$$
\left|f_{h}(t)\right|=\left|\int_{0}^{h t} e^{i \tau} d \tau\right| \leq|h||t| .
$$




\section{Coarse index theory and positive scalar curvature}

So certainly $\left|f_{h}(t)\right| \leq|F(t)|$ for $F$ being a constant multiple of the identity function, provided the variable $h$ is bounded. In particular, $\mathfrak{d o m}(F(D))=$ $\mathfrak{d o m}(D)$. Also,

$$
\left|f_{h}(t)\right| \leq C|h|
$$

for $|t| \leq C$ whenever $C>0$, so that $f_{n} \rightarrow 0$ uniformly on compact subsets and thus $f_{n}(D) u \rightarrow 0$ for every $u \in \mathfrak{d o m}(D)$ by (viii) of Theorem 1.3.3. Thus $\frac{1}{2 \pi} \int_{\mathbb{R}} \hat{\varphi}(s)\langle U(s) u, v\rangle d s$ exists as an integral of a continuous function on a compact interval.

By the Fourier-Inversion Theorem one has $\varphi(t)=\frac{1}{2 \pi} \int_{-R}^{+R} \hat{\varphi}(s) e^{i s t} d s$. Furthermore, there exists a sequence $\left(\tau_{n}\right)_{n \in \mathbb{N}}$ of step functions which uniformly converges to $\hat{\varphi}$. Since the sequence $\left(f_{n}\right)_{n \in \mathbb{N}}$ with $f_{n}(s):=e^{i s t} \tau_{n}$ of gap continuous functions converges uniformly to the function given by $s \mapsto \hat{\varphi}(s) e^{i s t}$, we have

$$
\varphi(t)=\frac{1}{2 \pi} \int_{-R}^{+R} \hat{\varphi}(s) \exp (i s t) d t=\frac{1}{2 \pi} \lim _{n \rightarrow \infty} \int_{-R}^{+R} \tau_{n}(s) e^{i s t} d s
$$

for any $t \in \mathbb{R}$. Set $\varphi_{n}(s):=\frac{1}{2 \pi} \int_{-R}^{+R} \tau_{n}(s) e^{i s t} d s$. Let $u \in \mathscr{H}$. We claim $\varphi_{n}(D) u \rightarrow \varphi(D) u$. This follows from the following estimate

$$
\begin{aligned}
\left\|\left(\varphi(D)-\varphi_{n}(D)\right) u\right\| & =\left\|\frac{1}{2 \pi}\left(\int_{-R}^{+R}\left(\hat{\varphi}(s)-\tau_{n}(s)\right) e^{i s D} d s\right) u\right\| \\
& \leq \frac{1}{2 \pi} \int_{-R}^{+R}\left|\hat{\varphi}(s)-\tau_{n}(s)\right|\left\|e^{i s D} u\right\| d s \\
& =\frac{1}{2 \pi} \int_{-R}^{+R}\left|\hat{\varphi}(s)-\tau_{n}(s)\right|\|u\| d s .
\end{aligned}
$$

From this we can conclude

$$
\begin{aligned}
\langle\varphi(D) u, v\rangle & =\lim _{n \rightarrow \infty}\left\langle\varphi_{n}(D) u, v\right\rangle \\
& =\lim _{n \rightarrow \infty}\left\langle\frac{1}{2 \pi}\left(\int_{-R}^{+R} \tau_{n}(s) e^{i s D} d s\right) u, v\right\rangle \\
& =\lim _{n \rightarrow \infty} \frac{1}{2 \pi} \int_{-R}^{+R} \tau_{n}(s)\left\langle e^{i s D} u, v\right\rangle d s \\
& =\frac{1}{2 \pi} \int_{-R}^{+R}\left\langle\hat{\varphi}(s) e^{i s t} u, v\right\rangle d s .
\end{aligned}
$$

Here we have used for the second inequality the fact that

$$
\left(\int_{a}^{b} A(s) d s\right) \cdot u=\int_{a}^{b}(A(s) \cdot u) d s
$$

for $u \in E$, whenever $A:[a, b] \rightarrow \mathscr{L}(E)$ is a continuous path of bounded operators on a Banach space $E$.

(v) That $\varphi(D)$ is a locally compact operator for $\varphi \in C_{0}(\mathbb{R})$ can be proven along the lines of [21, Prop. 10.5.2]. 


\section{Coarse index theory and positive scalar curvature}

We are left to prove that $\varphi(D)$ is a finite propagation operator. This will follow from the finite propagation property of the operator $U(s)=e^{i s D}$ as follows. First of all, let us assume $\varphi \in W$ and $\operatorname{supp}(\hat{\varphi}) \subset[-R, R]$. In this case we can compute

$$
\begin{aligned}
\langle\rho(f) \varphi(D) \rho(g) u, v\rangle & =\left\langle\varphi(D) \rho(g) u, \rho(f)^{*} v\right\rangle \\
& =\int_{-R}^{+R} \hat{\varphi}(s)\left\langle U(s) \rho(g) u, \rho(f)^{*} v\right\rangle d s \\
& =\int_{-R}^{+R} \hat{\varphi}(s)\langle\rho(f) U(s) \rho(g) u, v\rangle d s .
\end{aligned}
$$

whenever $f, g \in C_{0}(M)$. From this the desired result follows since $U(s)$ has propagation $|s|$.

According to Lemma 1.3.4 there exists for every $\varphi \in C_{0}(\mathbb{R})$ a sequence $\left(\varphi_{n}\right)$ in $W$ which converges uniformly to $\varphi$, and Part (ix) of Lemma 1.3.3 implies $\varphi_{n}(D) \rightarrow \varphi(D)$ in norm. Thus also $\varphi(D) \in \mathrm{C}^{*}(\mathscr{H}, \rho)$ in this case.

\subsubsection{Definition of the coarse indices}

Let $\mathrm{D}^{*}(\mathscr{H}, \rho)$ be the $\mathrm{C}^{*}$-algebra generated by all controlled and pseudolocal $T \in \mathscr{L}_{A}^{*}(\mathscr{H})$. The $\mathrm{C}^{*}$-algebra $\mathrm{C}^{*}(\mathscr{H}, \rho)$ is an ideal in the unital $\mathrm{C}^{*}$-algebra $\mathrm{D}^{*}(\mathscr{H}, \rho)$ and we can consider the associated six term exact sequence in $\mathrm{K}$ theory

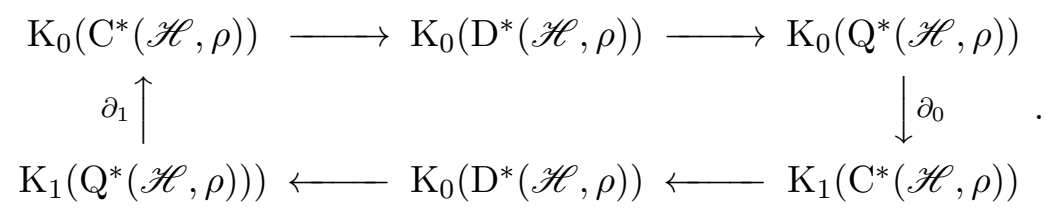

Here we have written $\mathrm{Q}^{*}(\mathscr{H}, \rho)$ for $\mathrm{D}^{*}(\mathscr{H}, \rho) / \mathrm{C}^{*}(\mathscr{H}, \rho)$ for reasons of space.

For $S, T \in \mathrm{D}^{*}(\mathscr{H}, \rho)$ we write $S \sim T$ if $S-T \in \mathrm{C}^{*}(\mathscr{H}, \rho)$.

Let $\chi \in C^{\infty}(\mathbb{R})$ be a normalizing function, i.e. an odd function $\chi \in C^{\infty}(\mathbb{R})$ with $\chi(0)=0$ and $\lim _{t \rightarrow \pm \infty} \chi(t)= \pm 1$. Set

- $\varphi:=1-\chi^{2} \in C_{0}(\mathbb{R})$ and

- $\bar{\chi}:=(1+\chi) / 2$.

By part (v) of Theorem 1.3.5 we have

$$
\bar{\chi}(D)^{2}=\left(\frac{1+2 \chi+\chi^{2}}{4}\right)(D)=\left(\frac{1+2 \chi+(1-\varphi)}{4}\right)(D) \sim \bar{\chi}(D) .
$$

Hence $\bar{\chi}(D)$ defines a projection in $\mathrm{Q}^{*}(\mathscr{H}, \rho)$ and consequently an element in the $\mathrm{K}_{0}$-group of this quotient $\mathrm{C}^{*}$-algebra. Analogously, if $\mathscr{H}=\mathscr{H}_{0} \oplus \mathscr{H}_{1}$ and $\rho=\rho_{0} \oplus \rho_{1}$ is in addition graded such that

$$
D=\left[\begin{array}{cc}
0 & D_{1} \\
D_{0} & 0
\end{array}\right]
$$


with respect to this grading, and $U: \mathscr{H}_{0} \rightarrow \mathscr{H}_{1}$ is a unitary isomorphism which covers $^{9}$ the identity $\operatorname{id}_{M}: M \rightarrow M$, then it can be shown that $U^{*} \chi\left(D_{0}\right): \mathscr{H}_{0} \rightarrow \mathscr{H}_{0}$ defines a unitary element in the quotient $\mathrm{C}^{*}$-algebra $\mathrm{Q}^{*}\left(\mathscr{H}_{0}, \rho_{0}\right)$ and therefore an element in the $\mathrm{K}_{1}$-group of this $\mathrm{C}^{*}$-algebra. Using these elements we define the coarse index as follows:

1.3.6 Definition (Coarse index). Let $\chi \in C^{\infty}(\mathbb{R})$ be a normalizing function and $\bar{\chi}:=(1+\chi) / 2$. The coarse index of $D$ is the following class:

$$
\operatorname{ind}_{p}(D):= \begin{cases}\partial_{1}\left[U^{*} \chi\left(D_{0}\right)\right] & , p=0 \\ \partial_{0}[\bar{\chi}(D)] & , p=1 .\end{cases}
$$

We call $\operatorname{ind}_{0}(D) \in \mathrm{K}_{0}\left(\mathrm{C}^{*}(\mathscr{H}, \rho)\right)$ and $\operatorname{ind}_{1}(D) \in \mathrm{K}_{0}\left(\mathrm{C}^{*}\left(\mathscr{H}_{0}, \rho_{0}\right)\right)$ the even and odd index of $D$, respectively.

One can show that the indices $\operatorname{ind}_{p}(D) \in \mathrm{K}_{p}\left(\mathrm{C}^{*}(M ; A)\right.$ (for $\left.p=0,1\right)$ are welldefined, that is, they do neither depend on the particular choice of the normalizing function $\chi$ nor the choice of the unitary $U: \mathscr{H}_{0} \rightarrow \mathscr{H}_{1}$ covering the identity. For details we refer the interested reader to [38].

The following Theorem 1.3.7 shows that the coarse indices of a Dirac operator $D$ are obstructions to the invertibility of $D$ (acting on $H^{1}(M, S)$ ). Using the Lichnerowicz formula it follows that the coarse indices are obstructions to the existence of uniform positive scalar curvature on the underlying manifold, see Theorem 1.3.9. In Section 1.4.6 of this chapter we show that even more is true: the coarse index is an obstruction to the existence of a metric which is uniformly positive outside a compact subset. This will be important for applications, see the proof of Theorem 1.6.9 later in this chapter.

1.3.7 Theorem (Coarse index and spectrum). If $\operatorname{ind}_{p}(D) \neq 0$, then the following holds for the spectrum of $D$ :

1. Graded case $(p=0)$ : One has $0 \in \sigma(D)$.

2. Ungraded case $(p=1)$ : One has $\sigma(D)=\mathbb{R}$.

Or, to put it negatively:

1'. Graded case $(p=0)$ : One has $\operatorname{ind}_{0}(D)=0$ if $D$ is invertible.

2'. Ungraded case $(p=1)$ : One has $\operatorname{ind}_{1}(D)=0$ if $D+\lambda I$ is invertible for some $\lambda \in \mathbb{R}$

Proof. 1. Graded case: Assume $0 \notin \sigma(D)$. Then there exists a normalizing function $\chi$, which only takes the values \pm 1 on $\sigma(D)$. Hence $\chi(D)^{2}=I$ and we can compute as follows

$$
\begin{aligned}
{\left[\begin{array}{cc}
0 & \chi\left(D_{0}\right)^{*} \\
\chi\left(D_{0}\right) & 0
\end{array}\right]\left[\begin{array}{cc}
0 & \chi\left(D_{0}\right)^{*} \\
\chi\left(D_{0}\right) & 0
\end{array}\right] } & =\left[\begin{array}{cc}
\chi\left(D_{0}\right)^{*} \chi\left(D_{0}\right) & 0 \\
0 & \chi\left(D_{0}\right) \chi\left(D_{0}\right)^{*}
\end{array}\right] \\
& =\left[\begin{array}{cc}
I_{0} & 0 \\
0 & I_{1}
\end{array}\right] .
\end{aligned}
$$

${ }^{9}$ Cf. [21, Def. 6.9.3] for the this. 
Hence $U^{*} \chi\left(D_{0}\right)$ is unitary and thus defines an element in $\mathrm{K}_{1}\left(\mathrm{D}^{*}\left(\mathscr{H}_{0}, \rho_{0}\right)\right)$. Because of the exactness of the six-term exact sequence we finally obtain

$$
\operatorname{ind}_{0}(D)=\left(\partial_{1} \circ \mathrm{K}_{1}(\pi)\right)\left[U^{*} \chi\left(D_{0}\right)\right]=0 .
$$

2. Ungraded case: Assume there exists $x_{0} \in \mathbb{R}$ with $x_{0} \notin \sigma(D)$. Since $\sigma(D)$ is closed and whence the resolvent set $\rho(D)$ is open, there exists $\varepsilon>0$ with $\left(x_{0}-\varepsilon, x_{0}+\varepsilon\right) \subset \rho(D)$, and one can find a normalizing function $\chi \in C(\mathbb{R})$, which is constantly -1 left of $x_{0}-\varepsilon$ and constantly +1 on the right of $x_{0}+\varepsilon$. Hence $\chi(D)^{2}=I$, as $\chi^{2}=1$ on $\sigma(D)$. Therefore $\chi(D)$ is an involution in the Roe algebra $\mathrm{C}^{*}(\mathscr{H}, \rho)$, which defines a $\mathrm{K}_{0}$-class

$$
\left[\frac{\chi(D)+I}{2}\right] \in \mathrm{K}_{0}\left(\mathrm{C}^{*}(\mathscr{H}, \rho)\right) .
$$

Because of the exactness of the six term exact sequence we thus obtain

$$
\operatorname{ind}_{0}(D)=\left(\partial_{0} \circ K_{0}(\pi)\right)[\chi(D)]=0 .
$$

This completes the proof.

1.3.8 Lemma. Let $\mathscr{H}$ be a Hilbert A-module. Assume $T: \operatorname{dom}(T) \rightarrow \mathscr{H}$ is an self-adjoint regular operator with domain in $\mathscr{H}$ such that for some $C>0$ the inequality

$$
\|T u\| \geq C\|u\|
$$

holds for any $u \in \mathfrak{d o m}(T)$. Then $T$ admits a bounded inverse.

Proof. We give a proof along the lines of [29, Lemma 3.1] and use the functional calculus for $T$ presented in Theorem 1.3.3. Suppose $0 \in \sigma(T)$. Choose a function $f \in C_{0}(\mathbb{R})$ with $f(t)=0$ for $|t| \geq C / 2$ which has $f(0)=1$ as its maximal absolute value. Then the function $g \in C_{0}(\mathbb{R})$ with $g(t)=t f(t)$ is such that $\|g\|_{\infty} \leq C / 2$. Therefore $\|g(T)\| \leq C / 2$ by (iv) of Theorem 1.3.3. Furthermore, one has $\|f(T)\|=1$ by part (iv) of Theorem 1.3.3. Therefore we can choose $u \in \mathscr{H}$ such that $\|f(T) u\|>1 / 2$. This implies by part (iii) of Theorem 1.3.3 that

$$
\|T f(T) u\|=\|g(T) u\| \leq \frac{C}{2}<C\|f(T) u\| .
$$

On the other hand $\|T f(T) u\| \geq C\|f(T) u\|$ by assumption.

1.3.9 Theorem (Coarse index as obstruction to uniform positive scalar curvature). Let $(M, g)$ be a complete Riemannian spin manifold and

$$
D: \Gamma(M, \Sigma M) \rightarrow \Gamma(M, \Sigma M)
$$

the spinorial Dirac operator, and $E$ be a flat Hilbert A-module bundle over $M$. If the scalar curvature scal of $M$ satisfies $\mathrm{scal} \geq C>0$ for some $C>0$, then the Roe indices $\operatorname{ind}_{p}\left(D_{E}\right)$ (for $\left.p=0,1\right)$ of the twisted Dirac operator $D_{E}$ vanish. 
Proof. We set $S=\Sigma M \otimes E$. Because $E$ is flat, the Lichnerowicz formula takes the form

$$
D_{E}^{2}=\Delta^{S}+\frac{\text { scal }_{4}}{4} \mathrm{id}_{S}
$$

Thus for every section $u \in \Gamma_{\text {cpt }}(M, S)$ one has (because $D_{E}$ is formally selfadjoint)

$$
\begin{aligned}
\left\|D_{E} u\right\|^{2} & =\left\langle D_{E} u, D_{E} u\right\rangle \\
& =\left\langle D_{E}^{2} u, u\right\rangle \\
& =\left\langle\Delta^{S} u, u\right\rangle+\left\langle\frac{\operatorname{scal}}{4} u, u\right\rangle \\
& =\|\nabla u\|^{2}+\int_{\operatorname{supp}(u)} \frac{\operatorname{scal}(x)}{4}\|u(x)\|^{2} d x \\
& \geq\|\nabla u\|^{2}+\frac{C}{4}\|u\|^{2} \\
& \geq \frac{C}{4}\|u\|^{2} .
\end{aligned}
$$

More generally, one knows [16, Thm. 2.8] that $\mathfrak{d o m}\left(\bar{D}_{E}\right)$ is the closure of $\Gamma_{\text {cpt }}(M, S)$ with respect to the Sobolev 1-norm $\|u\|_{1}^{2}=\int_{M}\langle u(x), u(x)\rangle+$ $\langle\nabla u(x), \nabla u(x)\rangle d x$ and

$$
\left\|\bar{D}_{E} u\right\|_{L^{2}(M, S)}^{2}=\|\bar{\nabla} u\|_{L^{2}(M, S)}^{2}+\int_{M} \frac{\operatorname{scal}(x)}{4}\|u(x)\|^{2} d x
$$

for each $u \in \mathfrak{d o m}\left(\bar{D}_{E}\right)$. From this the claim follows from Lemma 1.3.8 applied to the operator $\bar{D}_{E}: H^{1}(M, S) \rightarrow L^{2}(M, S)$ on $L^{2}(M, S)$.

\subsection{A vanishing theorem for the coarse indices}

\subsubsection{Introduction}

In this section we prove the following theorem (see Theorem 1.4.28):

Vanishing Theorem: Let $(M, g)$ be an non-compact and complete Riemannian spin manifold. Assume that $K \subset M$ is a compact subset such that the scalar curvature of the metric is uniformly positive outside of $K$. Then $\operatorname{ind}_{p}(D) \in \mathrm{K}_{p}\left(\mathrm{C}^{*}\left(L^{2}(M, S)\right)\right.$ vanishes for $p=0,1$. Here $D: \Gamma(M, S) \rightarrow \Gamma(M, S)$ with $S=\Sigma M \otimes \mathscr{V}_{M}$ is the twisted Dirac operator obtained by twisting the spinorial Dirac operator of $(M, g)$ with the Mishchenko line bundle $\left(\mathscr{V}_{M}, \nabla^{\mathscr{V}} M\right)$.

This kind of result was first obtained (independently) by Roe and $\mathrm{Yu}$ for the (untwisted) spinorial Dirac operator ${ }^{10}$. See [36, Ch. 5, p. 68] and [49]. Originally, both the proof by Roe as well as the one by $\mathrm{Yu}$ make use of a result

\footnotetext{
${ }^{10}$ The result is part of Yu's thesis from 1991. He writes in [49] that he obtained a preprint version of Roe's Memoirs article [36] shortly afterwards. Yu's article was received 1991 by the publisher but was only published in 1997, which explains the time difference between the publication of [36] and [49]
} 


\section{Coarse index theory and positive scalar curvature}

due to Gromov and Lawson on the finite-dimensionality of the $L^{2}$-kernel of the spinorial Dirac operator on a Riemannian spin manifold whose scalar curvature is uniformly positive outside of a compact subset. We briefly review this result and two of its proofs in Subsection 1.4.2. Since an analogous result is not available for the twisted Dirac operator, it became necessary to find another proof for the vanishing theorem which would not rely on such a result. Nevertheless, the main steps in proving the vanishing theorem will be essentially the same (let $A$ be the group $\mathrm{C}^{*}$-algebra of the fundamental group of $M$ ):

1. Step: The map induced in K-theory by the inclusion $i: \mathscr{K}_{A}\left(L^{2}(M, S)\right) \rightarrow$ $\mathrm{C}^{*}(M ; A)$ vanishes.

2. Step: The element in $\mathrm{K}_{p+1}\left(\mathrm{C}^{*}(M, A) / \mathrm{D}^{*}(M, A)\right)$ that is mapped to $\operatorname{ind}_{p}(D)$ under the boundary map $\partial_{p+1}$ in the six-term exact sequence can be lifted to $\mathrm{K}_{p+1}\left(\mathrm{D}^{*}(M, A) / \mathscr{K}_{A}\left(L^{2}(M, S)\right)\right)$.

From this the vanishing theorem will follow by the naturality of the six-term exact sequence. The first step will be put in practice in Subsection 1.4.4 after collecting some useful material on Hilbert $\mathrm{C}^{*}$-modules in Subsection 1.4.3, and in full generality in 1.4.5. The first proof is an adaptation of the one already given by $\mathrm{Yu}$ [49] for the (untwisted) spinorial Dirac operator and the second, more general one, uses an argument from [22]. For both proofs, the main part of the argument is an 'infinity swindle' which makes use of the existence of a geodesic ray in the non-compact and complete Riemannian manifold $(M, g)$. The adaptation of Yu's proof, described in Subsection 1.4.4, uses the notion of orthonormal bases for Hilbert $\mathrm{C}^{*}$-modules and Kasparov's Stabilization Theorem. We summarize this and other preparatory material in Subsection 1.4.3. In this subsection we will also show that $L^{2}(M, S)$ admits a countable orthonormal basis and is thus isomorphic to the standard Hilbert $A$-module $\ell^{2}(A)$. This will allow us in the sequel to identify the K-theory groups of $\mathscr{K}_{A}\left(L^{2}(M, S)\right)$ with those of $A$. In the final Subsection 1.4.6 we show that the function $I-\chi(D)^{2}$ of the twisted Dirac operator lies in the ideal $\mathrm{C}_{K}^{*}(M ; A)$ of $\mathrm{C}^{*}(M ; A)$ for at least one normalizing function $\chi$, provided the scalar curvature on the underlying Riemannian manifold is uniformly positive outside of a compact subset $K \subset M$. This immediately gives the second of the steps outlined above as will be explained in more detail at the end of Subsection 1.4.6.

\subsubsection{Finite-dimensionality of the $L^{2}$-kernel}

In [16] Gromov and Lawson gave two independent proofs for the fact that the $L^{2}$-kernel

$$
\operatorname{ker} D^{*}=\operatorname{ker} \bar{D} \subset H^{1}(M, S)
$$

of the spinorial Dirac operator $D$ on a complete non-compact Riemannian manifold is finite-dimensional provided the scalar curvature of the manifold is uniformly positive outside a compact subset. Since this means that the projection onto the kernel of the Dirac operator is a compact operator, this result implies that the Dirac operator has vanishing coarse index. We set out to review 
these results here and study whether they remain true if one twists with the Mishchenko line bundle.

The following result is taken from [16, Theorem 3.2, p. 326].

1.4.1 Theorem (Gromov and Lawson). Let $M$ be a complete Riemannian manifold and $D: \Gamma(M, S) \rightarrow \Gamma(M, S)$ a generalized Dirac operator. If there exists a compact subset $K \subset M$ and a constant $\kappa_{0}>0$ such that the scalar curvature satisfies $\kappa \geq \kappa_{0}$ outside of $K$, then the $L^{2}$-kernel

$$
\begin{aligned}
\operatorname{ker} \bar{D} & =\left\{u \in L^{2}(M, S) ; D u=0 \text { (weakly) }\right\} \\
& =\left\{u \in L^{2}(M, S) ;\left\langle u, D^{*} \varphi\right\rangle=0, \forall \varphi \in \Gamma_{\mathrm{cpt}}(M, S)\right\}
\end{aligned}
$$

is finite-dimensional. (Here $\bar{D}$ is the unique self-adjoint extension of D.) In particular, if the fundamental group $\Gamma:=\pi_{1}(M)$ of $M$ is finite, then the $L^{2}$ kernel of $D_{\mathscr{V}_{M}}$, the Dirac operator of $M$ twisted by the Mishchenko line bundle over $M$, is finite-dimensional.

Proof. Let $u \in L^{2}(M, S)$ with $D u=0$. By elliptic regularity $u$ is smooth. If we choose $\kappa_{1}>0$ such that $\kappa_{1} \geq-\kappa / 4$ on $K$, we can make the following estimate (detailed derivation follows)

$$
\|\nabla u\|^{2}+\kappa_{0}\|u\|_{M \backslash K}^{2} \leq \kappa_{1}\|u\|_{K}^{2} .
$$

Derivation: $\kappa \geq \kappa_{0}$ in $M \backslash K$ implies

$$
\begin{aligned}
0 & =\left\langle D^{2} u, u\right\rangle \\
& =\|\nabla u\|^{2}+\left\langle\frac{\kappa}{4} u, u\right\rangle \\
& =\|\nabla u\|^{2}+\int_{K}\left\langle\frac{\kappa}{4} u, \sigma\right\rangle_{x} d x+\int_{M \backslash K}\left\langle\frac{\kappa}{4} u, u\right\rangle_{x} d x \\
& \geq\|\nabla u\|^{2}+\int_{K}\left\langle\frac{\kappa}{4} u, u\right\rangle_{x} d x+\kappa_{0} \int_{M \backslash K}\langle u, u\rangle_{x} d x
\end{aligned}
$$

which gives the desired inequality. From $\|u\|_{M}^{2}=\|u\|_{K}^{2}+\|u\|_{M \backslash K}^{2}$. we obtain from the above inequality the following:

$$
\frac{1}{\kappa_{0}+\kappa_{1}}\|\nabla u\|^{2}+\frac{\kappa_{0}\|u\|_{M}^{2}}{\kappa_{0}+\kappa_{1}} \leq\|u\|_{K}^{2} .
$$

Let $\varepsilon>0$ and choose an $\varepsilon$-dense subset $\left\{x_{1}, \ldots, x_{m}\right\}$ of $K$. Set $H:=\operatorname{ker} \bar{D}$. We claim $\operatorname{dim}(H) \leq d$ with $d:=m r$, where $r:=2^{\langle n\rangle}$ is the rank of the spinor bundle of $M$. Suppose our claim is not true. Then there exists $u_{0} \in H \backslash\{0\}$ with $u_{0}\left(x_{k}\right)=0$ for $1 \leq k \leq d$. (Concerning this: There exists a linear independent subset $\left\{e_{1}, \ldots, e_{d+1}\right\}$ of $\operatorname{ker} \bar{D}$. We make the ansatz $u_{0}=\sum_{j=1}^{d+1} \lambda_{j} e_{j}$ with $\lambda_{j} \in \mathbb{C}$. From this we obtain the following $m$ equations:

$$
0=u_{0}\left(x_{k}\right)=\sum_{j=1}^{d+1} \lambda_{j} e_{j}\left(x_{k}\right) \quad, \quad k=1, \ldots, m
$$


Over each of the balls $B\left(x_{1}, \varepsilon\right), \ldots, B\left(x_{m}, \varepsilon\right)$ we can identify sections of $\Sigma M$ with functions from the corresponding ball to $\mathbb{C}^{r}$. Having done this, the above $m$ equations yield a homogeneous system of linear equations with $d$ equations and $d+1$ unknowns. By the rank-nullity theorem a linear map from $\mathbb{C}^{d+1}$ to $\mathbb{C}^{d}$ has a non-trivial kernel. From this our claim follows.) Of course, we can assume $\left\|u_{0}\right\|_{M}=1$.

Let $\Omega$ be an open neighbourhood of $K$. By Friedrichs' Lemma we can find a constant $C>0$ such that

$$
\|u\|_{C^{1}, K} \leq C\|u\|_{\Omega}
$$

for each $u \in \Gamma(\Omega, S)$ with $D u=0$. From this and because $\left\{x_{1}, \ldots, x_{m}\right\}$ is $\varepsilon$-dense, one can conclude $\left\|u_{0}(x)\right\| \leq C \varepsilon$ for all $x \in K$. But this implies

$$
0<\frac{\kappa_{0}}{\kappa_{0}+\kappa_{1}} \leq\left\|u_{0}\right\|_{K}^{2} \leq C^{2} \varepsilon^{2} \operatorname{vol}(K)
$$

for any $\varepsilon>0$.

The following result is also taken from [16, Theorem 3.7, p. 327] and proves again that the $L^{2}$-kernel of the Dirac operator on a complete non-compact Riemannian manifold whose scalar curvature is uniformly positive outside of a compact subset, is finite dimensional.

1.4.2 Theorem (Gromov and Lawson). Let $M$ be a complete Riemannian manifold and assume that the scalar curvature is uniformly positive outside of a compact subset $K \subset M$. Then there exists a constant $C>0$ such that the Hilbert space sum

$$
H_{C}:=\bigoplus_{|\lambda| \leq C} E_{\lambda} \subset H^{1}(M, S)
$$

is finite-dimensional. Here $E_{\lambda}$ denotes the eigenspace of the operator $\bar{D}: H^{1}(M, S) \rightarrow L^{2}(M, S)$, corresponding to the eigenvalue $\lambda$. In particular $\operatorname{dim}\left(E_{0}\right)=\operatorname{dim} \operatorname{ker}(\bar{D})<\infty$.

Proof. Fix $\kappa_{0}, \kappa_{1}>0$ such that $-\kappa_{1} \leq \kappa / 4$ globally and $\kappa_{0} \leq \kappa / 4$ outside of $K$. To begin with, let $C>0$ be arbitrary at first. For $u \in H_{C}$ with $\bar{D} u=\lambda u$ where $|\lambda| \leq C$, it follows from the Lichnerowicz formula $D^{2}=\nabla^{*} \nabla+\frac{\kappa}{4}$ that

$$
\|\nabla u\|^{2}+\int \frac{\kappa(x)}{4}\|u(x)\|^{2} d x=\|\bar{D} u\|^{2} \leq C^{2}\|u\|^{2} .
$$

From this

$$
\|\nabla u\|^{2}+\kappa_{0}\|u\|_{M \backslash K}^{2} \leq C^{2}\|u\|^{2}+\kappa_{1}\|u\|_{K}^{2}
$$

follows. This implies

$$
\|\nabla u\|^{2}+\left(\kappa_{0}-C^{2}\right)\|u\|^{2} \leq\left(\kappa_{0}+\kappa_{1}\right)\|u\|_{K}^{2} .
$$

Therefore, if $C$ is such that $0<C^{2}<\kappa_{0}$, one has for $u \in H_{C} \backslash\{0\}$ :

$$
C^{\prime}:=\frac{\kappa_{0}-C^{2}}{\kappa_{0}+\kappa_{1}} \leq \frac{\|u\|_{K}^{2}}{\|u\|^{2}} .
$$


For the following consideration, let $C^{\prime \prime}>0$ be the square root of $C^{\prime}$, and $Q \in \Psi^{-1}(M, S)$ a parametrix for $D$ with remainder $R \in \Psi^{-\infty}(M, S)$. Thus

$$
\bar{Q} \bar{D}=I-\bar{R},
$$

where the operators $\bar{D}: H^{1}(M, S) \rightarrow L^{2}(M, S)$ and $\bar{Q}: L^{2}(M, S) \rightarrow L^{2}(M, S)$, as well as $\bar{R}: H^{1}(M, S) \rightarrow L^{2}(M, S)$ are the bounded extension given by $D, Q$ and $R$. Finally, let $\rho: L^{2}(M, S) \rightarrow L^{2}\left(K, S_{\mid K}\right)$ be the restriction operator and consider the compact operator $\tilde{R}=\rho \circ R$. For $u \in H_{C}$ we have $\|D u\| \leq C\|u\|$ and thus

$$
\begin{aligned}
\|\tilde{R} u\| & =\|\rho(I-Q D) u\| \\
& \geq\|u\|_{K}-\|Q D u\| \\
& \geq\|u\|_{K}-\|Q\|\|D u\| \\
& \geq\|u\|_{K}-C\|Q\|\|u\| \\
& \geq\left(C^{\prime \prime}-C\|Q\|\right)\|u\| .
\end{aligned}
$$

By choosing $C>0$ sufficiently small we obtain $\|\tilde{R} u\| \geq C^{\prime \prime \prime}\|u\|$ for some positive constant $C^{\prime \prime \prime}>0$. Since $\tilde{R}$ is compact, $H_{C}$ is finite-dimensional.

To obtain Yu's and Roe's vanishing theorem from the previous results one uses the following lemma.

1.4.3 Lemma (Spectral characterization of self-adjoint Fredholm operators). Let $H$ be a Hilbert space and $T: \operatorname{dom}(T) \rightarrow H$ be a (bounded or unbounded) operator defined on $\mathfrak{d o m}(T) \subset H$. Assume $0 \in \sigma(T)$. The followig are equivalent:

(i) The operator $T$ has discrete spectrum of finite multiplicity in a neighbourhood of zero, i.e. there exists $C>0$ such that $\operatorname{dim}\left(\bigoplus_{|\lambda| \leq C} E_{\lambda}\right)<\infty$ (the sum being a sum of Hilbert spaces).

(ii) The kernel $\operatorname{ker}(T)$ is finite-dimensional and $\operatorname{im}(T)$ is closed.

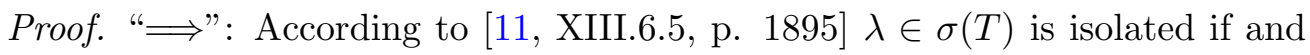
only if $\operatorname{im}(T-\lambda I)$ is closed. Since, by (i), the spectrum of $T$ is discrete near $\lambda=0$, the image of $T$ is closed. Since the spectrum of $T$ near 0 has finite multiplicity $\operatorname{ker} D \subset E_{0}$ is finite-dimensional.

"£": The point $\lambda=0$ is isolated in $\sigma(T)$ since $\operatorname{im}(T)$ is closed by (ii). Thus there exists $C>0$ such that $[-C, C] \cap \sigma(T)=\{0\}$ and $\operatorname{dim}\left(\bigoplus_{|\lambda| \leq C} E_{\lambda}\right)=$ $\operatorname{dim} \operatorname{ker}(T)<\infty$.

\subsubsection{Preliminary facts about Hilbert $\mathrm{C}^{*}$-modules}

In this section $A$ denotes a (complex) $\mathrm{C}^{*}$-algebra. Most of the time it will be assumed that $A$ is unital. But we will state this explicitly when needed. We denote the closed cone given by the positive elements in $A$ by $A_{+}$. A useful reference for the basic properties of $A_{+}$is [33, Section 2.2].

We recollect some facts about orthonormal bases in Hilbert $\mathrm{C}^{*}$-modules. 
1. Coarse index theory and positive scalar curvature

1.4.4 Definition (Orthonormal sequence, orthonormal basis). Let $A$ be a unital $\mathrm{C}^{*}$-algebra with unit element $1_{A}$ and let $\mathscr{H}$ be a Hilbert $A$-module.

1. An orthonormal sequence is a system $\left(e_{i}\right)_{i \in I}$ of vectors in $E$ such that $\left\langle e_{i}, e_{j}\right\rangle=\delta_{i j} \cdot 1_{A}$.

2. An orthonormal basis is an orthonormal sequence $\left(e_{i}\right)_{i \in I}$ which is complete in the sense that $x=\sum_{i \in I} e_{i}\left\langle e_{i}, x\right\rangle$ holds for each $x \in E$.

Also recall the following definition.

1.4.5 Definition (Countably generated). Let $A$ be a $\mathrm{C}^{*}$-algebra. A Hilbert $\mathrm{C}^{*}$ module $\mathscr{H}$ is called countably generated if there exists a sequence $\left(x_{i}\right)_{i \in \mathbb{N}}$ in $\mathscr{H}$ such that $\mathscr{H}=\varlimsup_{A}\left(x_{i}\right)_{i \in \mathbb{N}}$ is the closure of the set of all finite linear combinations in the $x_{i}$ with coefficients in $A$.

1.4.6 Definition (Direct sum of Hilbert $\mathrm{C}^{*}$-modules). Let $A$ be a $\mathrm{C}^{*}$-algebra. Let $\mathscr{H}$ and $\mathscr{H}^{\prime}$ be two Hilbert $A$-modules. Their direct sum $\mathscr{H} \oplus \mathscr{H}^{\prime}$ is a Hilbert $A$-module when equipped with the $A$-valued inner product given by

$$
\left\langle(e, f),\left(e^{\prime}, f^{\prime}\right)\right\rangle:=\left\langle e, e^{\prime}\right\rangle+\left\langle f, f^{\prime}\right\rangle .
$$

Recall that an isomorphism between Hilbert $\mathrm{C}^{*}$-modules is a linear bijection which preserves the inner products.

1.4.7 Theorem (Kasparov's stabilization theorem). Let $A$ be a $\mathrm{C}^{*}$-algebra. If $\mathscr{H}$ is a countably generated Hilbert A-module, then the direct sum of $\mathscr{H}$ with $\ell^{2}(A)$, as defined in Definition 1.4.6, is isomorphic as a Hilbert A-module to $\ell^{2}(A)$ :

$$
\mathscr{H} \oplus \ell^{2}(A) \cong \ell^{2}(A) .
$$

1.4.8 Corollary (Extending orthonormal bases). Let $A$ be a unital $\mathrm{C}^{*}$-algebra and let $\mathscr{H}$ be Hilbert $A$-submodule of $\ell^{2}(A)$. If $\left(e_{i}\right)_{i \in \mathbb{N}}$ is a countable orthonormal basis for $\mathscr{H}$, then there exists a countable orthonormal basis $\left(f_{j}\right)_{j \in \mathbb{N}}$ of $\ell^{2}(A)$ which extends the given orthonormal basis, i.e. one which is such that $\left\{e_{i} ; i \in \mathbb{N}\right\}$ is contained in $\left\{f_{j} ; j \in \mathbb{N}\right\}$.

Proof. We have $\mathscr{H}=\overline{\operatorname{lin}}_{A}\left(e_{i}\right)_{i \in \mathbb{N}}$. Using Kasparov's stabilization theorem we can identify $\ell^{2}(A)$ with $\mathscr{H} \oplus \ell^{2}(A)$. The elements of the standard orthornormal basis for $\ell^{2}(A)$ together with the elements of the given orthonormal basis of $\mathscr{H}$ define a countable orthonormal basis $\left(f_{j}\right)_{j \in \mathbb{N}}$ for $\mathscr{H} \oplus \ell^{2}(A)$ and hence for $\ell^{2}(A)$ via the above identification.

The following convergence result will be used subsequently in the proof of Lemma 1.4.21.

1.4.9 Lemma. Let $A$ be a unital $\mathrm{C}^{*}$-algebra and $\mathscr{H}$ a Hilbert $A$-module. If $\left(f_{j}\right)_{j \in \mathbb{N}}$ is an orthonormal system in $\mathscr{H}$, and $\left(a_{j}\right)_{j \in \mathbb{N}}$ is sequence in $A_{+}$which is bounded by an element in $\mathbb{C} \cdot 1_{A}$, then the series

$$
\sum_{j \in \mathbb{N}}\left\langle f_{j}, x\right\rangle^{*} a_{j}\left\langle f_{j}, x\right\rangle
$$

converges in norm for each fixed $x \in \mathscr{H}$. 
For later application in the proof of Lemma 1.4.21 we remark the following: This lemma applies in particular if the sequence $\left(a_{j}\right)_{j \in \mathbb{N}}$ is constant with the only value being a projection $p \in \operatorname{Proj}(A)$. In this case $p \leq 1$ by [33, Thm. 2.3 .2 , p. 50].

Proof of the lemma. By Corollary 1.4.8 we can choose an orthonormal basis $\left(e_{k}\right)_{k \in \mathbb{N}}$ of $\mathscr{H}$ which contains $\left(f_{j}\right)_{j \in \mathbb{N}}$. We can assume that this sequence is enumerated such that $e_{2 j}=f_{j}$ for any $j \in \mathbb{N}$. From $x=\sum_{k=1}^{\infty} e_{k}\left\langle e_{k}, x\right\rangle$ the equality $\langle x, x\rangle_{H}=\sum_{k=1}^{\infty}\left\langle e_{k}, x\right\rangle^{*}\left\langle e_{k}, x\right\rangle$ follows. In particular, it follows that the latter series converges. Next choose $b \in \mathbb{C} \cdot 1_{A}$ with $a_{j} \leq b$ for all $j \in \mathbb{N}$. Then by $[33$, Theorem $2.2 .5,(3)$, p. 46$]$

$$
\left\|\sum_{j=n}^{\infty}\left\langle f_{j}, x\right\rangle^{*} a_{j}\left\langle f_{j}, x\right\rangle\right\|_{A} \leq\|b\|_{A}\left\|\sum_{k=2 n}^{\infty}\left\langle e_{k}, x\right\rangle^{*}\left\langle e_{k}, x\right\rangle\right\|_{A} .
$$

From this the lemma follows with the help of [48, Corollary on p. 237].

We recall the definition of a compact operator (in the sense of Kasparov) on a Hilbert $\mathrm{C}^{*}$-module. It reads as follows.

1.4.10 Definition (Elementary compact operator, generalized compact operator). Let $\mathscr{H}$ be a Hilbert $A$-module. The operator $\Theta_{x, y}: \mathscr{H} \rightarrow \mathscr{H}$ given by $\Theta_{x, y}(z):=x\langle y, z\rangle$ is called an elementary compact operator. The $\mathrm{C}^{*}$ subalgebra of $\mathscr{L}_{A}^{*}(\mathscr{H})$ generated by the elementary compact operators is denoted by $\mathscr{K}_{A}(\mathscr{H})$. The elements of $\mathscr{K}_{A}(\mathscr{H})$ are called generalized compact operators (in the sense of Kasparov).

Let $A$ be a unital $\mathrm{C}^{*}$-algebra and $S$ a bundle of Hilbert $A$-modules over the manifold $M$.

1.4.11 Definition (The Hilbert $A$-module of square-integrable sections). We define $L^{2}(M, S)$ as the closure of $\Gamma_{\mathrm{cpt}}(M, S)$ with respect to the norm $\|\cdot\|_{L^{2}(M, S)}$ induced by the following $A$-valued inner product:

$$
\langle s, t\rangle:=\int_{M}\langle s(x), t(x)\rangle_{S_{x}} d x .
$$

In the following we will work with a technical assumption which reads as follows.

1.4.12 Assumption. Let $(M, g)$ be a Riemannian manifold, with or without boundary, which is compact or non-compact, and let $p: S \rightarrow M$ be a hermitian bundle of Hilbert $A$-modules with typical fibre $A^{r}$, where $r \in \mathbb{N}$, the free $A$ module of rank $r \in \mathbb{N}$. Typically we will consider $S=\Sigma M \otimes \mathscr{V}_{M}$ with its typical fibre $\mathbb{C}^{\langle n\rangle} \otimes \mathrm{C}^{*} \pi=\left(\mathrm{C}^{*} \pi\right)^{\langle n\rangle}$.

Later in this chapter we will need an explicit description for the K-theory groups of the compact operators on $L^{2}(M, S)$. For this we show that $L^{2}(M, S)$ is isomorphic as a Hilbert $A$-module to the standard Hilbert $A$-module $\ell^{2}(A)$. This gives the required description since the K-theory groups of $\mathscr{K}_{A}\left(\ell^{2}(A)\right)$ are known to be those of $A$.

Recall that an isomorphism between Hilbert $A$-modules is a linear bijection which preserves the inner products. 
1.4.13 Lemma. Let $\left(U_{j}\right)_{j \in \mathbb{N}}$ be a partition of $M$ into simplices given by a triangulation of $M$. Choose vector bundle charts $\psi_{j}: p^{-1}\left(U_{j}\right) \rightarrow U_{j} \times A^{r}$ (fibrewise these are isomorphisms of Hilbert A-modules), which trivialize the bundle over the simplices. Then the map

$$
h: L^{2}(M, E) \rightarrow \bigoplus_{j \in \mathbb{N}} L^{2}\left(U_{j}, U_{j} \times A^{r}\right) \quad, \quad h(s):=\sum_{j \in \mathbb{N}}^{\oplus} \psi_{j} \circ\left(\chi_{j} s\right)
$$

is an isomorphism of Hilbert A-modules. Here $\chi_{j}$ is the characteristic function of $U_{j}$.

Proof. We begin by remarking that the bundle $U_{j} \times A^{r}$ over $U_{j}$ is equipped with the metric induced by the metric $\langle\cdot, \cdot\rangle_{*}$ on $A^{r}$ given by

$$
\left\langle\left(a_{1}, \ldots, a_{r}\right),\left(b_{1}, \ldots, b_{r}\right)\right\rangle_{*}=\sum_{j=1}^{r} a_{j} b_{j}^{*} .
$$

Let $s, t \in \Gamma(M, S)$. Then one has

$$
\begin{aligned}
\langle s, t\rangle & =\int_{M}\langle s(x), t(x)\rangle d x \\
& =\sum_{j \in \mathbb{N}} \int_{U_{j}}\left\langle\chi_{j}(x) s(x), \chi_{j}(x) t(x)\right\rangle d x \\
& =\sum_{j \in \mathbb{N}} \int_{U_{j}}\left\langle\psi_{j}\left(\chi_{j}(x) s(x)\right), \psi_{j}\left(\chi_{j}(x) t(x)\right)\right\rangle d x \\
& =\sum_{j \in \mathbb{N}}\left\langle\psi_{j} \circ\left(\chi_{j} s\right), \psi_{j} \circ\left(\chi_{j} t\right)\right\rangle \\
& =\langle h(s), h(t)\rangle .
\end{aligned}
$$

Thus $h$ preserves the inner products. Cleary, $h$ is also surjective: if we are given sections $s_{j} \in L^{2}\left(U_{j}, U_{j} \times A^{r}\right)$ for each $j$, then we can first of all extend $\psi_{j}^{-1} \circ s_{j}$ trivially to the section $\chi_{j}\left(\psi_{j}^{-1} \circ s_{j}\right)$. Then $s=\sum \chi_{j}\left(\psi_{j}^{-1} \circ s_{j}\right)$ is mapped onto $\left(s_{j}\right)_{j \in \mathbb{N}}$ by $h$.

If $U \subset M$ is an open subset of $M$, we can identify $L^{2}\left(U, U \times A^{r}\right)$, the completion of $\Gamma_{\mathrm{cpt}}\left(U, U \times A^{r}\right)$ with respect to $\|\cdot\|_{L^{2}\left(U, U \times A^{r}\right)}$, with the completion $L_{*}^{2}\left(U, A^{r}\right)$ of $C_{\mathrm{cpt}}^{\infty}\left(U, A^{r}\right)$ with respect to the norm $\|\cdot\|_{L_{*}^{2}\left(U, A^{r}\right)}$ induced by the $A$-valued inner product

$$
\langle f, g\rangle_{L_{*}^{2}\left(U, A^{r}\right)}=\int_{U}\langle f(x), g(x)\rangle_{*} d x
$$

This notation is chosen in this way to distinguish the above norm from the norm $\|\cdot\|_{2}$ on the Banach space $L^{2}\left(U, A^{r}\right)$ of square-integrable, $A^{r}$-valued functions on $U$.

Let $\otimes$ denote the tensor product of complex vector spaces. For $r \in \mathbb{N}$ the space $\mathbb{C}^{r} \otimes A$ becomes a Hilbert $A$-module via $(e \otimes a) b=e \otimes(a b)$ and $\left\langle e_{1} \otimes a_{1}, e_{2} \otimes a_{2}\right\rangle=$ $\left\langle e_{1}, e_{2}\right\rangle\left\langle a_{1}, a_{2}\right\rangle$. Clearly, this Hilbert $A$-module is isomorphic to $A^{r}$. In the 
following it will be more convenient to work with $\mathbb{C}^{r} \otimes A$ instead of $A^{r}$. Let $U \subset M$ be an open subset. We view $C\left(U, \mathbb{C}^{r}\right) \otimes A$ as a subspace of $C\left(U, \mathbb{C}^{r} \otimes A\right)$ by regarding the function $f \otimes a$ as the function with $(f \otimes a)(x)=f(x) \otimes a$ in $x \in U$.

1.4.14 Remark. We observe the following: if $f, g \in L^{2}\left(U, \mathbb{C}^{r}\right)$ and $a, b \in A$, then

$$
\langle f \otimes a, g \otimes b\rangle_{L^{2}\left(U, \mathbb{C}^{r} \otimes A\right)}=\int_{U}\langle f(x), g(x)\rangle\langle a, b\rangle_{*} d x=\langle f, g\rangle_{L^{2}\left(U, \mathbb{C}^{r}\right)}\langle a, b\rangle_{*}
$$

by the definition of the scalar product on $\mathbb{C}^{r} \otimes A$. In particular, if $\left(f_{j}\right)_{j \in \mathbb{N}}$ is a sequence in $C_{\mathrm{cpt}}^{\infty}\left(U, \mathbb{C}^{r}\right)$, which converges in mean to $f \in C_{\mathrm{cpt}}^{\infty}\left(U, \mathbb{C}^{r}\right)$ then, for any $a \in A$, the sequence $\left(f_{j} \otimes a\right)_{j \in \mathbb{N}}$ of functions in $C_{\mathrm{cpt}}^{\infty}\left(U, \mathbb{C}^{r} \otimes A\right)$ converges to $f \otimes a$ in the norm-topology of $L_{*}^{2}\left(U, \mathbb{C}^{r} \otimes A\right)$. This allows us to define $f \otimes a \in L_{*}^{2}\left(U, \mathbb{C}^{r} \otimes A\right)$ for any $f \in L_{*}^{2}\left(U, \mathbb{C}^{r}\right)$ and $a \in A$.

1.4.15 Lemma. Let $A$ be a unital $\mathrm{C}^{*}$-algebra with unit element $1_{A}$ and $U \subset M$ an open subset of $M$. Furthermore, let $r \in \mathbb{N}$. Choose an orthonormal basis $\left(e_{j}\right)_{j \in \mathbb{N}}$ for the Hilbert space $L^{2}\left(U, \mathbb{C}^{r}\right)$. Then $\left(e_{j} \otimes 1_{A}\right)_{j \in \mathbb{N}}$ is an orthonormal basis for the Hilbert $A$-module $L^{2}\left(U, \mathbb{C}^{r} \otimes A\right)$. In particular, $L^{2}\left(U ; \mathbb{C}^{r} \otimes A\right)$ is isomorphic to $\ell^{2}(A)$ as a Hilbert $A$-module.

Proof. First of all, we note that by the above Remark 1.4.14 $\left(e_{j} \otimes 1_{A}\right)_{j \in \mathbb{N}}$ is an orthonormal system in $L_{*}^{2}\left(U, \mathbb{C}^{r} \otimes A\right)$. To see that this system is an orthornormal basis it suffices to show that $\left(e_{j}\right)_{j \in \mathbb{N}}$ has dense $A$-linear span in $C_{\mathrm{cpt}}^{\infty}\left(U, \mathbb{C}^{r} \otimes A\right)$ where the closure is being taken with respect to the norm-topology of $L_{*}^{2}\left(U, \mathbb{C}^{r} \otimes\right.$ $A)$. Let $f \in C_{\mathrm{cpt}}^{\infty}\left(U, \mathbb{C}^{r} \otimes A\right)$ and set $K:=\operatorname{supp}(f)$. We denote by $C_{K}^{\infty}\left(U, \mathbb{C}^{r}\right)$ the space of all smooth functions with compact support in $K$, equipped with the topology of uniform convergence on $K$. Clearly, convergence to 0 in $C_{K}^{\infty}\left(U, \mathbb{C}^{r}\right)$ implies convergence to 0 in $L^{2}\left(U, \mathbb{C}^{r}\right)$. Therefore the proof will be complete if we show that $f$ is a uniform limit of functions in $C_{K}^{\infty}\left(U, \mathbb{C}^{r}\right) \otimes A$ since by Remark 1.4.14 the $A$-linear closure of $\left(e_{j}\right)_{j \in \mathbb{N}}$ is dense in this space in the norm-topology of $L_{*}^{2}\left(U, \mathbb{C}^{r} \otimes A\right)$. Let $\varepsilon>0$. Since $f$ is continuous and $K$ is compact we can choose points $x_{1}, \ldots, x_{k}$ such that for each $x \in K$ one has $\left\|f(x)-f\left(x_{j}\right)\right\|_{\mathbb{C}^{r} \otimes A}<\varepsilon$ for some $1 \leq j \leq k$. The open sets

$$
V_{j}:=\left\{x \in K ;\left\|f(x)-f\left(x_{j}\right)\right\|_{\mathbb{C}^{r} \otimes A}<\varepsilon\right\} \quad, \quad 1 \leq j \leq k
$$

cover $K$. Let $\left(\pi_{j}\right)_{1 \leq j \leq k}$ be a partition of unity subordinated to this open covering of $K$. Then

$$
\begin{aligned}
\left\|f(x)-\sum_{j=1}^{k} \pi_{j}(x) f\left(x_{j}\right)\right\|_{\mathbb{C}^{r} \otimes A} & =\left\|\sum_{j=1}^{k} \pi_{j}(x)\left(f(x)-f\left(x_{j}\right)\right)\right\|_{\mathbb{C}^{r} \otimes A} \\
& \leq \sum_{j=1}^{k} \pi_{j}(x)\left\|\left(f(x)-f\left(x_{j}\right)\right)\right\|_{\mathbb{C}^{r} \otimes A} \\
& <\varepsilon,
\end{aligned}
$$

which gives the desired result. 
From Lemma 1.4.13 and Lemma 1.4.15 we conclude the following corollary.

1.4.16 Corollary. The Hilbert $A$-module $L^{2}(M, E)$ is isomorphic to $\ell^{2}(A)$ for $\operatorname{dim}(M) \geq 1$.

\subsubsection{The vanishing of $K_{0}(i)$ : First proof}

1.4.17 Definition (Geodesic ray). Let $(M, g)$ be a Riemannian manifold. A geodesic ray in $M$ is a geodesic

$$
\gamma: \mathbb{R}_{+} \rightarrow M
$$

which minimizes the distance between any pair of points on itself, i.e.

$$
d\left(\gamma\left(t_{0}\right), \gamma\left(t_{1}\right)\right)=\int_{t_{0}}^{t_{1}}\left\|\gamma^{\prime}(t)\right\| d t=v\left(t_{1}-t_{0}\right)
$$

where $v$ is the constant velocity of $\gamma$.

We can always assume $v=1$.

Thus every restriction $\gamma_{\mid[a, b]}:[a, b] \rightarrow M$ of $\gamma$ to a compact subinterval $[a, b]$ of $\mathbb{R}_{+}$is a geodesic segment. The content of the following lemma, is that there always exists a geodesic ray in the given context.

1.4.18 Lemma (Existence of rays). In every complete and non-compact Riemannian manifold $(M, g)$ there exists at least one geodesic ray.

Proof. A proof for the existence of rays in the above setting can be found, for example, in [9, p. 51 and p. 135] or in [13, p. 92, Proposition 2.95].

1.4.19 Assumption. From now on we assume that $(M, g)$ is complete, noncompact, and of positive injectivity radius $\operatorname{inj}(M, g)>0$. Finally, choose $r>0$ with $r \leq \operatorname{inj}(M, g)$.

Let $p: S \rightarrow M$ be as in Assumption 1.4.12. The following remark is essentially the key to the proof of the subsequent Lemma 1.4.20: Suppose $s, t \in \Gamma_{\mathrm{cpt}}(M, S)$ and let $\varphi: \pi^{-1}(U) \rightarrow U \times A^{r}$ and $\psi: \pi^{-1}(V) \rightarrow V \times A^{r}$ be isometric trivializations of $S$ over sets $U$ and $V$ with $\operatorname{supp}(s) \subset U$ and $\operatorname{supp}(t) \subset V$. Then there exists functions $\bar{s}, \bar{t} \in C_{\mathrm{cpt}}^{\infty}\left(M, A^{r}\right)$ with $s(x)=\varphi^{-1}(x, \bar{s}(x))$ and $t(x)=\psi^{-1}(x, \bar{t}(x))$. One then has the following equality $(*)$

$$
\begin{aligned}
\langle s, t\rangle_{L^{2}(M, S)} & =\int_{M}\langle s(x), t(x)\rangle_{S_{x}} d x \\
& =\int_{M}\langle\bar{s}(x), \bar{t}(x)\rangle_{A^{r}} d x \\
& =\int_{M} \sum_{i=1}^{r}\left(\bar{s}^{i}(x)\right)\left(\bar{t}^{i}(x)\right)^{*} d x .
\end{aligned}
$$

The following lemma will be useful in the sequel. 
1.4.20 Lemma. Under the above Assumption 1.4.19, if $\gamma: \mathbb{R}_{+} \rightarrow M$ is a geodesic ray in $M$, then there exists a sequence $\left(f_{j}\right)_{j \in \mathbb{N}}$ in $\Gamma_{\mathrm{cpt}}(M, S)$ such that the following hold:

(i) $\operatorname{supp}\left(f_{j}\right) \subset B_{j}$, where $B_{j}:=B(\gamma(j r) ; r / 2)$. (Note that these balls are disjoint since we assume $v=1$, and that each ball is diffeomorphic to $\mathbb{R}^{n}$, $n=\operatorname{dim}(M)$, since $r \leq \operatorname{inj}(M, g)$ by our assumptions.)

(ii) $\left\langle f_{i}, f_{j}\right\rangle_{L^{2}(M, S)}=\delta_{i, j} \cdot 1_{\mathrm{C}^{*} \pi}$ in $\mathrm{C}^{*} \pi$.

(iii) $\left\langle g f_{j}, g f_{j}\right\rangle_{L^{2}(M, S)} \in \mathbb{C} \cdot 1_{\mathrm{C}^{*} \pi}$ whenever $g \in C(M)$.

Proof. Such a sequence of sections $f_{j} \in \Gamma_{\mathrm{cpt}}(M, S)$ can be constructed by first choosing a sequence of trivializations $\varphi_{i}: \pi^{-1}\left(U_{i}\right) \rightarrow U_{i} \times A^{r}$ of the bundle over sets $U_{j} \subset B_{j}$ and then choosing $\bar{f}_{j} \in C_{\mathrm{cpt}}^{\infty}\left(M,\left(\mathbb{C i}_{\mathrm{C}^{*} \pi}\right)\right)$ with $f_{j}(x)=\varphi_{j}^{-1}\left(x, \bar{f}_{j}(x)\right)$ using the above equality $(*)$ in such a way that (ii) holds. Property (i) is fulfilled by the choice of the $U_{j}$ as subset of $B_{J}$ and (iii) holds by $(*)$ and the choice of the $\bar{f}_{j}$ as scalar-valued functions.

1.4.21 Lemma. Under the above Assumption 1.4.19, if $p \in \operatorname{Proj}(A)$ is a projection and if $\left(f_{j}\right)_{j \in \mathbb{N}} \subset L^{2}(M, S)$ is a sequence like in Lemma 1.4.20, then $\mathrm{K}_{0}(i)$ maps $\left[\Theta_{f_{1} p, f_{1} p}\right]$ to zero.

Proof. (i) We use the notation of Definition 1.4.10 and consider the sequence $\left(\Theta_{f_{j} p, f_{j} p}\right)_{j \in \mathbb{N}}$. Obviously, the following relations hold:

$$
\Theta_{f_{j} p, f_{j} p}^{*}=\Theta_{f_{j} p, f_{j} p}^{2}=\Theta_{f_{j} p, f_{j} p}
$$

and

$$
\Theta_{f_{j} p, f_{j} p} \Theta_{f_{k} p, f_{k} p}=\delta_{j k} \Theta_{f_{j} p, f_{k} p}
$$

for all $j, k \in \mathbb{N}$. For any $N \in \mathbb{N}$ set

$$
T_{N}:=\sum_{j=1}^{N} \Theta_{f_{j} p, f_{j+1} p}=\sum_{j=1}^{N} f_{j} p\left\langle f_{j+1}, \cdot\right\rangle .
$$

We claim that the strong $\operatorname{limit} T:=\mathrm{s}-\lim _{N \rightarrow \infty} T_{N}$ exists. This can be proven as follows: Let $u \in L^{2}(M, S)$ and $M, N \in \mathbb{N}$ with $N>M$. We calculate:

$$
\begin{aligned}
\left\|\left(T_{N}-T_{M}\right) u\right\|_{L^{2}(M, S)}^{2} & =\left\|\sum_{j=M+1}^{N} f_{j} p\left\langle f_{j+1}, u\right\rangle\right\|_{L^{2}(M, S)}^{2} \\
& =\left\|\sum_{j=M+1}^{N}\left\langle f_{j+1} p, u\right\rangle_{L^{2}(M, S)}^{*}\left\langle f_{j+1} p, u\right\rangle_{L^{2}(M, S)}\right\|_{A} \\
& =\left\|\sum_{j=M+1}^{N}\left\langle f_{j+1}, u\right\rangle_{L^{2}(M, S)}^{*} p\left\langle f_{j+1}, u\right\rangle_{L^{2}(M, S)}\right\|_{A} .
\end{aligned}
$$

Hence, by Lemma 1.4.9 and the remark following it, $\left(T_{N} s\right)_{n \in \mathbb{N}}$ is a Cauchy sequence and therefore convergent. 


\section{Coarse index theory and positive scalar curvature}

(ii) In this part of the proof we show that $T$ is an element of $\mathrm{C}_{\mathrm{Roe}}^{*}(M ; A)$. First of all, $T$ is adjointable with adjoint given by

$$
T^{*}=\sum_{j=1}^{\infty} \Theta_{f_{j} p, f_{j+1} p}^{*}=\sum_{j=1}^{\infty} \Theta_{f_{j+1} p, f_{j} p} .
$$

Hence $T \in \mathscr{L}_{A}^{*}\left(L^{2}(M, S)\right)$. To see that $T$ is locally compact we proceed as follows. Let $g \in C_{0}(M)$. According to Corollary 1.4 .8 we can complete $\left(f_{j}\right)_{j \in \mathbb{N}}$ to an orthonormal basis $\left(e_{k}\right)_{k \in \mathbb{N}}$ of $L^{2}(M, S)$. One has

$$
\left\|T g-T_{N} g\right\|=\sup _{\|u\|=1}\left\|(T g) u-\left(T_{N} g\right) u\right\|_{L^{2}\left(M, \mathscr{V}_{M}\right)} .
$$

Since

$$
(T g) u-\left(T_{N} g\right) u=\sum_{j=N+1}^{\infty} f_{j} p\left\langle f_{j+1}, g u\right\rangle
$$

holds for any $u \in L^{2}(M, S)$, we find using Lemma 1.4.20 (ii)

$$
\begin{aligned}
\left\|(T g) u-\left(T_{N} g\right) u\right\|_{L^{2}(M, S)}^{2} & =\left\|\left\langle\sum_{j=N+1}^{\infty} f_{j} p\left\langle f_{j+1}, g u\right\rangle, \sum_{k=N+1}^{\infty} f_{k} p\left\langle f_{k+1}, g u\right\rangle\right\rangle\right\|_{A} \\
& =\left\|\sum_{j, k=N+1}^{\infty}\left\langle f_{j+1}, g u\right\rangle^{*} p\left\langle f_{j}, f_{k}\right\rangle p\left\langle f_{k+1}, g u\right\rangle\right\|_{A} \\
& =\left\|\sum_{j=N+1}^{\infty}\left\langle f_{j+1}, g u\right\rangle^{*} p\left\langle f_{j+1}, g u\right\rangle\right\|_{A} .
\end{aligned}
$$

Now Lemma 1.4.9 shows that $T_{N} g \rightarrow T g$ not only pointwise but also uniformly. Thus $T g$ is compact. Analogously, $g T$ is compact: by definition

$$
\left\|g T-g T_{N}\right\|=\sup _{\|u\|=1}\left\|\left(g T-g T_{N}\right) u\right\|_{L^{2}(M, S)} .
$$

One finds

$$
(g T) u-\left(g T_{N}\right) u=\sum_{j=N+1}^{\infty} g f_{j} p\left\langle f_{j+1}, u\right\rangle
$$

for any $u \in L^{2}(M, S)$. Here we have used that the multiplication by $g$ is a continuous operator. An auxiliary computation shows:

$$
\begin{aligned}
\left\|(g T) u-\left(g T_{N}\right) u\right\|_{L^{2}(M, S)}^{2} & =\left\|\sum_{j, k=N+1}^{\infty}\left\langle g f_{j} p\left\langle f_{j+1}, u\right\rangle, g f_{k}\left\langle f_{k+1}, u\right\rangle\right\rangle\right\|_{A} \\
& =\left\|\sum_{j, k=N+1}^{\infty}\left\langle f_{j+1}, u\right\rangle^{*}\left\langle g f_{j} p, g f_{k} p\right\rangle\left\langle f_{k+1}, u\right\rangle\right\|_{A} \\
& =\left\|\sum_{j, k=N+1}^{\infty}\left\langle f_{j+1}, u\right\rangle^{*} p\left\langle g f_{j}, g f_{k}\right\rangle p\left\langle f_{k+1} u\right\rangle\right\|_{A} \\
& =\left\|\sum_{j=N+1}^{\infty}\left\langle f_{j+1}, u\right\rangle^{*} p\left\langle g f_{j}, g f_{j}\right\rangle p\left\langle f_{j+1}, u\right\rangle\right\|_{A} .
\end{aligned}
$$


By Lemma 1.4.20 the sequence $\left\langle g f_{j+1}, g f_{j+1}\right\rangle \in \mathbb{C} \cdot 1_{A}$ converges to zero as $j \rightarrow$ $\infty$ since $g \in C_{0}(M)$. Thus the sequence $\left(a_{j}\right)_{j \in \mathbb{N}}$ with $a_{j}:=p\left\langle g f_{j}, g f_{j}\right\rangle p \in A_{+}$of positive elements is bounded by an element in $\mathbb{C} \cdot 1_{A}$. Because of this, it follows from Lemma 1.4.9 that $g T_{N} \rightarrow g T$ not only pointwise but also uniformly.

Finally, we show that $T$ has finite propagation. Concerning this: we have to show that there exists $R>0$ such that $\operatorname{supp}(T u)$, for any $u \in \Gamma_{\text {cpt }}(M, S)$, is contained in the $R$-ball $B(\operatorname{supp}(u) ; R)$. To this end assume that $x \in M$ is such that

$$
0 \neq(T u)(x)=\sum_{j=1}^{\infty} f_{j}(x) p\left\langle f_{j+1}, u\right\rangle .
$$

This means that not all summands $f_{j}(x) p\left\langle f_{j+1}, u\right\rangle$ in the RHS are zero. Hence there exists $j_{0} \in \mathbb{N}$ such that $f_{j_{0}}(x) \neq 0$ and $\left\langle f_{j_{0}+1}, u\right\rangle \neq 0$. The latter condition implies that there exists a point $x_{0}$ in $B\left(j_{0}+1\right)$ such that $u\left(x_{0}\right) \neq 0$, i.e. $x_{0} \in$ $\operatorname{supp}(u)$. Since $\gamma$ is a geodesic ray with velocity $v=1$ we have $d\left(\gamma\left(j_{0} r\right), \gamma\left(\left(j_{0}+\right.\right.\right.$ 1) $r))=r$. Hence, if we set $R:=2 r$, then

$$
\begin{aligned}
d\left(x, x_{0}\right) & \leq d\left(x, \gamma\left(j_{0} r\right)\right)+d\left(\gamma\left(j_{0} r\right), x_{0}\right) \\
& \leq \frac{r}{2}+d\left(\gamma\left(j_{0} r\right), x_{0}\right) \\
& \leq \frac{r}{2}+d\left(\gamma\left(j_{0} r\right), \gamma\left(\left(j_{0}+1\right) r\right)\right)+d\left(\gamma\left(\left(j_{0}+1\right) r\right), x_{0}\right) \\
& \leq \frac{r}{2}+\frac{r}{2}+d\left(\gamma\left(j_{0} r\right), \gamma\left(\left(j_{0}+1\right) r\right)\right)=R .
\end{aligned}
$$

Thus $\operatorname{supp}(T u) \subset B(\operatorname{supp}(u) ; R)$ proving that $T$ has finite propagation.

(iii) A calculation shows:

$$
P:=T^{*} T=\sum_{j=2}^{\infty} \Theta_{f_{j} p, f_{j} p}
$$

and

$$
Q:=T T^{*}=\sum_{j=1}^{\infty} \Theta_{f_{j} p, f_{j} p} .
$$

Obviously, $P$ and $Q$ are self-adjoint and a calculation shows $P^{2}=P$ and $Q^{2}=Q$. Thus $P$ and $Q$ are projections in $\mathrm{C}_{\mathrm{Roe}}^{*}(M ; A)$ which are Murrayvon Neumann equivalent. Therefore one has in $\mathrm{K}_{0}\left(\mathrm{C}_{\text {Roe }}^{*}(M ; A)\right)$

$$
\begin{aligned}
{\left[T T^{*}\right] } & =\left[T^{*} T+\Theta_{f_{1} p, f_{1} p}\right] \\
& =\left[T^{*} T\right]+\left[\Theta_{f_{1} p, f_{1} p}\right] .
\end{aligned}
$$

Here we have used [Rørdam et al., Prop. 3.1.7, (iv)]. Now, by definition, $\left[T^{*} T\right]=\left[T T^{*}\right]$. Hence, $\left[\Theta_{f_{1} p, f_{1} p}\right]=0$ in $\mathrm{K}_{0}\left(\mathrm{C}_{\text {Roe }}^{*}(M ; A)\right)$.

Next we recall the stabilization theorem for K-theory. 
1.4.22 Remark (The stabilization map in K-Theory). Let $A$ be a $\mathrm{C}^{*}$-algebra, $H$ a separable Hilbert space and $\mathscr{K}_{\mathbb{C}}(H)$ the $\mathrm{C}^{*}$-algebra of all compact operators on $H$. The stabilization by $\mathscr{K}_{\mathbb{C}}(H)$ is the spatial tensor product of $\mathscr{K}_{\mathbb{C}}(H)$ and $A$. Denote by $e \in \mathscr{K}_{\mathbb{C}}(H)$ any rank-1-projection, i.e. an elementary compact operator $\Theta_{x, x}(z)=\langle z, x\rangle x$ where $x \in H$ with $\langle x, x\rangle=1$. The map

$$
A \rightarrow \mathscr{K}_{\mathbb{C}}(H) \otimes A \quad, \quad a \mapsto e \otimes a
$$

is a ${ }^{*}$-homorphism. The map induced by it on even K-theory

$$
\sigma_{A}^{H}: \mathrm{K}_{0}(A) \rightarrow \mathrm{K}_{0}\left(\mathscr{K}_{\mathbb{C}}(H) \otimes A\right) \quad, \quad[p] \mapsto[e \otimes p]
$$

is independent of the choice of $e$ and is called the stabilization map for $H$. It is a (non-trivial) theorem that $\sigma_{A}^{H}$ is an isomorphism. A similar statement holds for odd K-theory. The usual identification of $\mathscr{K}_{\mathbb{C}}(H) \otimes \mathbb{C}$ with $\mathscr{K}_{\mathbb{C}}(H)$ given by scalar multiplication gives an identification of $\mathrm{K}_{0}(\mathbb{C})$ with $\mathrm{K}_{0}\left(\mathscr{K}_{\mathbb{C}}(H)\right)$ :

$$
\mathrm{K}_{0}(\mathbb{C}) \stackrel{\sigma_{\mathbb{C}}}{\longrightarrow} \mathrm{K}_{0}\left(\mathscr{K}_{\mathbb{C}}(H) \otimes \mathbb{C}\right) \rightarrow \mathrm{K}_{0}\left(\mathscr{K}_{\mathbb{C}}(H)\right) \quad, \quad[1] \mapsto[e \otimes 1] \mapsto[e] .
$$

The choice of an orthonormal basis $\left(e_{j}\right)_{j \in \mathbb{N}}$ and $\left(f_{j}\right)_{j \in \mathbb{N}}$ for $L^{2}(M, S)$ and $L^{2}(M, \mathbb{C})$, respectively, induces the isometric embedding

$$
\iota: L^{2}(M, \mathbb{C}) \cong \ell^{2}(\mathbb{C}) \hookrightarrow \ell^{2}(A) \cong L^{2}(M, S)
$$

given by

$$
\sum_{j \in \mathbb{N}} \sigma_{j} f_{j} \mapsto\left(\sigma_{j}\right)_{j \in \mathbb{N}} \mapsto\left(\sigma_{j} \cdot 1_{A}\right)_{j \in \mathbb{N}} \mapsto \iota(f):=\sum_{j \in \mathbb{N}} \sigma_{j} e_{j} .
$$

1.4.23 Lemma. Let $p_{1}, p_{2}, p_{3}, \ldots \in A$ be projections such that the abelian group $\mathrm{K}_{0}(A)$ is generated by $\left\{\left[p_{1}\right],\left[p_{2}\right],\left[p_{3}\right], \ldots\right\}$, If $f \in L^{2}(M, \mathbb{C})$ with $\|f\|_{L^{2}(M, \mathbb{C})}=$ 1 , then the even $K$-theory classes of $\Theta_{\iota(f) p_{j}, \iota(f) p_{j}}, j=1,2,3, \ldots$, generate $\mathrm{K}_{0}\left(\mathscr{K}_{A}\left(L^{2}(M, S)\right)\right)$.

Proof. By [23, Lemma 1.2.6 and its proof, p. 24] the map

$$
U: L^{2}(M, \mathbb{C}) \otimes_{s} A \rightarrow L^{2}(M, S) \quad, \quad\left(\sigma_{j}\right)_{j \in \mathbb{N}} \otimes a \mapsto\left(\sigma_{j} \cdot 1_{A}\right)_{j \in \mathbb{N}} \cdot a
$$

is an isomorphism of Hilbert $A$-modules. Let

$$
\mu: \mathscr{K}_{A}\left(L^{2}(M, \mathbb{C}) \otimes_{s} A\right) \rightarrow \mathscr{K}_{A}\left(L^{2}(M, S)\right) \quad, \quad \Theta_{x, y} \mapsto \Theta_{U(x), U(y)}
$$

be the identification of $\mathrm{C}^{*}$-algebras induced by $U$. According to [23, Lemma 1.2 .7$, p. 24] the map

$$
\lambda: \mathscr{K}_{\mathbb{C}}\left(L^{2}(M, \mathbb{C})\right) \otimes A \rightarrow \mathscr{K}_{A}\left(L^{2}(M, \mathbb{C}) \otimes_{s} A\right) \quad, \quad \lambda\left(\Theta_{x, y} \otimes b c^{*}\right)=\Theta_{x \otimes b, y \otimes c}
$$

is an isomorphism of $\mathrm{C}^{*}$-algebras. Therefore the composition $(*)$ of the maps in the subsequent diagram is an isomorphism:

$$
\begin{aligned}
\mathrm{K}_{0}(A) \stackrel{\sigma_{A}^{L^{2}(M, \mathbb{C})}}{\longrightarrow} & \mathrm{K}_{0}\left(\mathscr{K}_{\mathbb{C}}\left(L^{2}(M, \mathbb{C})\right) \otimes A\right) \\
\stackrel{\mathrm{K}_{0}(\lambda)}{\longrightarrow} & \mathrm{K}_{0}\left(\mathscr{K}_{A}\left(L^{2}(M, \mathbb{C}) \otimes_{s} A\right)\right) \\
\stackrel{\mathrm{K}_{0}(\mu)}{\longrightarrow} & \mathrm{K}_{0}\left(\mathscr{K}_{A}\left(L^{2}(M, S)\right)\right.
\end{aligned}
$$


Choose the rank-1-projection which gives $\sigma_{A}^{L^{2}(M, \mathbb{C})}$ to be equal $\Theta_{f, f}$. Then under the above isomorphism $(*)$ the class $\left[p_{j}\right] \in \mathrm{K}_{0}(A)$ is mapped under the isomorphism as follows:

$$
\begin{aligned}
& {\left[p_{j}\right] \stackrel{\sigma_{A}^{L^{2}(M, \mathbb{C})}}{\longrightarrow}\left[\Theta_{f, f} \otimes p_{j}\right]} \\
& \stackrel{\mathrm{K}_{0}(\lambda)}{\longrightarrow} \quad\left[\Theta_{f \otimes p_{j}, f \otimes p_{j}}\right] \\
& \stackrel{\mathrm{K}_{0}(\mu)}{\longrightarrow} \quad\left[\Theta_{U\left(f \otimes p_{j}\right), U\left(f \otimes p_{j}\right)}\right]=\left[\Theta_{\iota(f) p_{j}, \iota(f) p_{j}}\right]
\end{aligned}
$$

This implies the claim.

1.4.24 Lemma. Choose $\left(f_{j}\right)_{j \in \mathbb{N}}$ as in Lemma 1.4.20. Then the map $\mathrm{K}_{0}(i)$

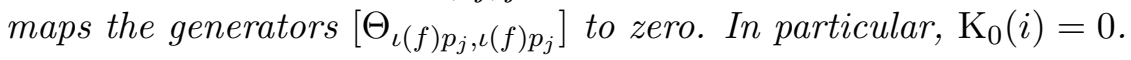

Proof. Complete $\left(f_{j}\right)_{j \in \mathbb{N}}$ to an orthonormal basis $\left(e_{k}\right)_{k \in \mathbb{N}}$ of $L^{2}(M, S)$. Then apply Lemma 1.4 .23 to $f \in L^{2}(M, \mathbb{C})$ with $\iota(f)=f_{1}$, to see that the classes $\left[\Theta_{f_{1} p_{j}, f_{1} p_{j}}\right]$ generate $\mathrm{K}_{0}\left(\mathscr{K}_{A}\left(L^{2}(M, S)\right)\right)$. From this the claim follows by Lemma 1.4.21.

\subsubsection{The vanishing of $\mathrm{K}_{0}(i)$ and $\mathrm{K}_{1}(i)$ : General proof.}

In the previous section we have shown that $\mathrm{K}_{0}(i)$ is zero for the inclusion map $i$ of the compact operators into the Roe $\mathrm{C}^{*}$-algebra. In this section we also prove that $\mathrm{K}_{1}(i)$ vanishes. Since the argument will also show that $\mathrm{K}_{0}(i)$ vanishes, we will obtain a second independent proof of this result. The argument used is an adaptation of well-known results from [8] and [22].

The following lemma will be useful in the proof of the next theorem.

1.4.25 Lemma. Let $\left(e_{j}\right)_{j \in \mathbb{N}}$ be an orthonormal basis of $L^{2}(M, S)$, and $\left(f_{j}\right)_{j \in \mathbb{N}}$ an orthonormal basis for $L^{2}(M, \mathbb{C})$. Furthermore, let $\iota: L^{2}(M, \mathbb{C}) \rightarrow L^{2}(M, S)$ be the isometric embedding given by $\iota\left(f_{j}\right)=e_{j}$ for each $j \in \mathbb{N}$. The following hold:

(i) The map $U: L^{2}(M, \mathbb{C}) \otimes_{s} A \rightarrow L^{2}(M, S)$ with $U(f \otimes a):=\iota(f) \cdot a$ is an isomorphism of Hilbert A-modules.

(ii) Define for each $u \in L^{2}(M, S)$ with $\langle u, u\rangle=1$ the *-homomorphism

$$
\Phi_{u}: A \rightarrow \mathscr{K}_{A}\left(L^{2}(M, S)\right) \quad, \quad a \mapsto \Theta_{u \cdot a, u} .
$$

Then $\Phi_{\iota(f)}$ induces an isomorphism in K-theory for each $f \in L^{2}(M, \mathbb{C})$ with $\|f\|=1$.

Proof. (i) Cf. [23, Lemma 1.2.6 and its proof, p. 24].

(ii) This was explained in the proof of Lemma 1.4.23.

1.4.26 Theorem. Let $(M, g)$ be a complete non-compact Riemannian spin manifold. Furthermore, let $S=\Sigma M \otimes \mathscr{V}$ be the spinor bundle of $M$ twisted by the Mishchenko line bundle of $M$. Then $\mathrm{K}_{p}(i)=0$ for $p=0,1$, where $i: \mathscr{K}_{A}\left(L^{2}(M, S)\right) \rightarrow \mathrm{C}^{*}(M ; A)$ is the inclusion map. 
Proof. (i) By Lemma 1.4.18 there exists a ray $\gamma: \mathbb{R}_{+} \rightarrow M$. Let $V: L^{2}\left(\mathbb{R}_{+}, A\right) \rightarrow L^{2}(M, S)$ be an isometry which covers ${ }^{11} \gamma$ and $f \in L^{2}(M, \mathbb{C})$ with $\|f\|=1$. The diagram

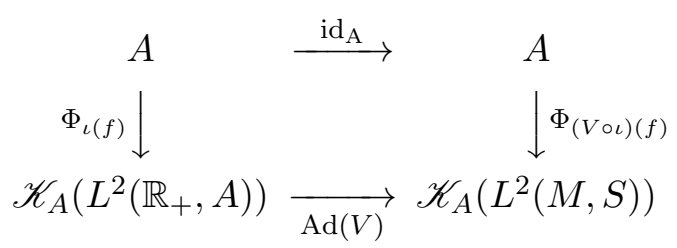

commutes and by Lemma 1.4.25 the vertical maps induce isomorphisms. Whence the map

$$
\gamma_{*}=\mathrm{K}_{p}(\operatorname{Ad}(V)): \mathrm{K}_{p}\left(\mathscr{K}_{A}\left(L^{2}\left(\mathbb{R}_{+}, A\right)\right)\right) \rightarrow \mathrm{K}_{p}\left(\mathscr{K}_{A}\left(L^{2}(M, S)\right)\right)
$$

is an isomorphism for $p=0,1$. From the commutative diagram

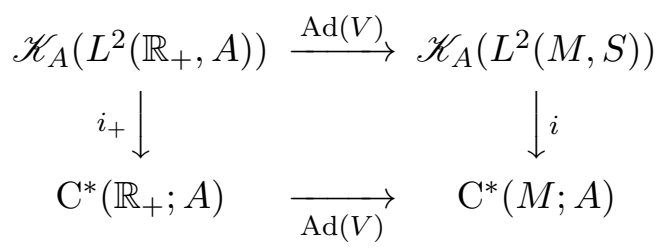

in which the vertical maps are the inclusion maps, we obtain the commutative diagramm

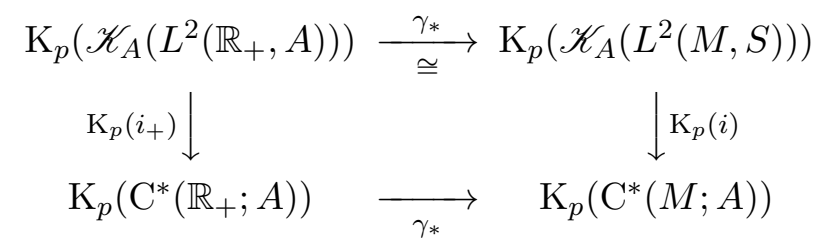

From this $\mathrm{K}_{p}(i)=0$ will follow by showing that $\mathrm{K}_{p}\left(\mathrm{C}^{*}\left(\mathbb{R}_{+} ; A\right)\right)$ vanishes. In order to show this we need to introduce various operators before we can complete the argument. This will be done in next part (ii) and after this preparation the proof will be completed in (iii).

(ii) First of all, set

$$
L^{2}\left(\mathbb{R}_{+}, A\right)_{\infty}:=\bigoplus_{j=1}^{\infty} L^{2}\left(\mathbb{R}_{+}, A\right)
$$

and let $U: L^{2}\left(\mathbb{R}_{+}, A\right) \rightarrow L^{2}\left(\mathbb{R}_{+}, A\right)_{\infty}$ be the isometric embedding given by

$$
U f:=(f \oplus 0 \oplus 0 \oplus \cdots) .
$$

The adjoint of $U$ is given by

$$
U^{*}\left(f_{1} \oplus f_{2} \oplus f_{3} \oplus \cdots\right)=f_{1} .
$$

We have $U^{*} U=I$ and $U U^{*}$ is the projection onto the first component. For the next step let $W: L^{2}\left(\mathbb{R}_{+}, A\right) \rightarrow L^{2}\left(\mathbb{R}_{+}, A\right)$ be the operator, which translates the

${ }^{11}$ See [22, Prop. 6.3.12, p. 150] for the existence of such an isometry. 
graph of a function one unit to the right side and extends the function trivially to the whole of $\mathbb{R}_{+}$, i.e.

$$
(W f)(t):= \begin{cases}f(t-1) & , t \geq 1 \\ 0 & , \text { otherwise. }\end{cases}
$$

Clearly, the adjoint of $W$ is given by left translation by one unit. Thus $\left(W^{*} f\right)(t)=f(t+1)$ for each $f \in L^{2}\left(\mathbb{R}_{+}, A\right)$ and $t \in \mathbb{R}_{+}$. We have $W^{*} W=I$ and $W W^{*}$ is the multiplication with the characteristic function $\chi_{[1, \infty)}$. Furthermore, the equalities

$$
\operatorname{supp}\left(W^{* j} f\right)=(\operatorname{supp}(f)-j) \cap \mathbb{R}_{+}
$$

and

$$
\operatorname{supp}\left(W^{j} f\right)=(\operatorname{supp}(f)+j)
$$

hold for each $j \in \mathbb{N}$. We notice that $M_{f} W=W M_{g}$ for $g=W^{*} f$. In particular, $\operatorname{supp}(g)=(\operatorname{supp}(f)-1) \cap \mathbb{R}_{+}$holds in this case.

In the next step we show that the operator

$$
\Psi(T):=\left(0 \oplus W T W^{*} \oplus W^{2} T W^{* 2} \oplus W^{3} T W^{* 3} \oplus \cdots\right)
$$

on $L^{2}\left(\mathbb{R}_{+}, A\right)_{\infty}$ is locally compact and finite propagation for each locally compact operator $T \in \mathscr{L}_{A}^{*}\left(\mathbb{R}_{+}, A\right)$ of finite propagation. To begin with, for any $\varphi, \psi \in C_{0}\left(\mathbb{R}_{+}\right)$we have

$$
\begin{aligned}
\rho(\varphi) \Psi(T) \rho(\psi) & =0 \oplus \bigoplus_{j=1}^{\infty} \rho(\varphi) W^{j} T W^{* j} \rho(\psi) \\
& =0 \oplus \bigoplus_{j=1}^{\infty} W^{j} \rho\left(\varphi_{j}\right) T \rho\left(\psi_{j}\right) W^{* j}
\end{aligned}
$$

for functions $\varphi_{j} \in C_{0}\left(\mathbb{R}_{+}\right)$and $\psi_{j} \in C_{0}\left(\mathbb{R}_{+}\right)$with $\operatorname{supp}\left(\varphi_{j}\right)=(\operatorname{supp}(\varphi)-j) \cap \mathbb{R}_{+}$ and $\operatorname{supp}\left(\psi_{j}\right)=(\operatorname{supp}(\psi)-j) \cap \mathbb{R}_{+}$. Since $T$ has finite propagation, this implies that $\Psi(T)$ also has finite propagation. Finally, we explain why $\Psi(T)$ is locally compact. First of all, we notice that

$$
\rho(f) \Psi(T)=\left(0 \oplus\left(\rho(f) W T W^{*}\right) \oplus\left(\rho(f) W^{2} T W^{* 2}\right) \oplus\left(\rho(f) W^{3} T W^{* 3}\right) \oplus \cdots\right)
$$

is an compact operator for each compactly supported function $f \in C_{0}\left(\mathbb{R}_{+}\right)$ simply because $\rho(f) W^{j}$ vanishes for $j$ sufficiently large and because $T$ is locally compact. Analogously, $\Psi(T) \rho(f)$ is compact. But then $\rho(f) \Psi(T)$ and $\Psi(T) \rho(f)$ are also compact for each $f \in C_{0}\left(\mathbb{R}_{+}\right)$.

In part (iii) we will use the isometry $V: L^{2}\left(\mathbb{R}_{+}, A\right)_{\infty} \rightarrow L^{2}\left(\mathbb{R}_{+}, A\right)_{\infty}$ given by

$$
V\left(f_{1} \oplus f_{2} \oplus f_{3} \oplus \cdots\right):=\left(0 \oplus W f_{1} \oplus W f_{2} \oplus \cdots\right) .
$$


Its adjoint is given by

$$
V^{*}\left(f_{1} \oplus f_{2} \oplus f_{3} \oplus \cdots\right)=\left(W^{*} f_{2} \oplus W^{*} f_{3} \oplus \cdots\right) .
$$

In (iii) we will use that $\operatorname{Ad}(V): \mathscr{L}_{A}^{*}\left(L^{2}\left(\mathbb{R}_{+}, A\right)_{\infty}\right) \rightarrow \mathscr{L}_{A}^{*}\left(L^{2}\left(\mathbb{R}_{+}, A\right)_{\infty}\right)$ maps $\mathrm{C}^{*}\left(\mathbb{R}_{+}, L^{2}\left(\mathbb{R}_{+}, A\right)\right)$ into itself. This can for instance be seen in the following way. If the operator $T \in \mathscr{L}_{A}^{*}\left(L^{2}\left(\mathbb{R}_{+}, A\right)_{\infty}\right)$ is locally compact and of finite propagation, then $V T V^{*}$ is also of this kind: to see that local compactness of $V T V^{*}$ let $\varphi \in C_{0}\left(\mathbb{R}_{+}\right)$. Then also $W^{*} \varphi \in C_{0}\left(\mathbb{R}_{+}\right)$and

$$
\rho(\varphi) V T V^{*}=V \rho\left(W^{*} \varphi\right) T V^{*}
$$

is compact since $\rho\left(W^{*} \varphi\right) T$ is compact and because of the fact that the compact operators form an ideal inside $\mathscr{L}_{A}^{*}\left(L^{2}\left(\mathbb{R}_{+}, A\right)_{\infty}\right)$. As $V T V^{*} \rho(\varphi)$ is compact if and only if $\rho(\varphi) V T^{*} V^{*}$ is compact, this shows that $V T V^{*}$ is locally compact. Finally, $V T V^{*}$ has finite propagation, because

$$
\begin{aligned}
\rho(\varphi) V T V^{*} \rho(\psi) & =(\rho(\varphi) V) T(\rho(\psi) V)^{*} \\
& =V \rho\left(W^{*} \varphi\right) T \rho\left(W^{*} \psi\right) V^{*}
\end{aligned}
$$

is zero if

$$
d\left(\operatorname{supp}\left(W^{*} \varphi\right), \operatorname{supp}\left(W^{*} \psi\right)\right)=d(\operatorname{supp}(\varphi), \operatorname{supp}(\psi)) \geq R
$$

where $R>0$ is choosen for $T$ as in (v) of Definition 1.2.5.

(iii) Consider the homomorphisms

$$
\Phi:=\operatorname{Ad}(U): \mathrm{C}^{*}\left(\mathbb{R}_{+}, L^{2}\left(\mathbb{R}_{+}, A\right)\right) \rightarrow \mathrm{C}^{*}\left(\mathbb{R}_{+}, L^{2}\left(\mathbb{R}_{+}, A\right)_{\infty}\right)
$$

and

$$
\Psi: \mathrm{C}^{*}\left(\mathbb{R}_{+}, L^{2}\left(\mathbb{R}_{+}, A\right)\right) \rightarrow \mathrm{C}^{*}\left(\mathbb{R}_{+}, L^{2}\left(\mathbb{R}_{+}, A\right)_{\infty}\right)
$$

given by

$$
\Phi(T):=\left(U T U^{*}:\left(f_{1} \oplus f_{2} \oplus f_{3} \oplus \cdots\right) \mapsto\left(T f_{1} \oplus 0 \oplus 0 \oplus \cdots\right)\right)
$$

and

$$
\Psi(T):=\left(0 \oplus W T W^{*} \oplus W^{2} T W^{* 2} \oplus W^{3} T W^{* 3} \oplus \cdots\right),
$$

respectively. Because of $U^{*} U=I, \mathrm{~K}_{p}(\Phi)$ is an injection for $p=0,1$. Obviously, $\Phi$ and $\Psi$ are orthogonal to each other. A straightforward computation shows that $\operatorname{Ad}(V) \circ(\Phi+\Psi)=\Psi$ holds. By [22, Lemma 3, p. 90] this implies the vanishing of $\mathrm{K}_{p}(\Phi)$ and thus $\mathrm{K}_{p}\left(\mathrm{C}^{*}\left(\mathbb{R}_{+}, A\right)\right)=0$ for $p=0,1$.

\subsubsection{The vanishing theorem}

In this final subsection of the chapter we prove the vanishing theorem. One half of the proof of this result uses the in the previous subsections established fact that the inclusion of the compact operators in the Roe $\mathrm{C}^{*}$-algebra induces the trivial map in K-theory, if the underlying complete Riemannian spin manifold is non-compact. The remaining part will use the following result which 
we establish next. We show that the relative Roe $\mathrm{C}^{*}$-algebra $\mathrm{C}_{K}^{*}(M ; A)$ of a Riemannian manifold $(M, g)$ with respect to a subset $K \subset M$ for which the scalar curvature of the metric is uniformly positive outside of $K$, contains the bounded transform $I-\chi(D)^{2}$ of the spinorial Dirac operator twisted with the Mishchenko line bundle for some normalizing function $\chi$. The idea for the construction of such a normalizing function $\chi$ is to decompose $f=\left(1-\chi^{2}\right)^{2}$ as $f=f_{\delta}+r_{\delta}$ and to use various estimates obtained from the functional calculus of $D$ as well as the Lichnerowicz formula to find out how to choose $f_{\delta}$ and $r_{\delta}$ appropriately in order to obtain a normalizing function as desired.

1.4.27 Theorem. Let $(M, g)$ be a complete Riemannian spin manifold and $K \subset M$ a subset such that the scalar curvature of the metric is uniformly positive outside $K$. Let $D: \Gamma(M, S) \rightarrow \Gamma(M, S)$ with $S=\Sigma M \otimes \mathscr{V}_{M}$ be the twisted Dirac operator obtained by twisting the spinorial Dirac operator of $(M, g)$ with the Mishchenko line bundle $\left(\mathscr{V}_{M}, \nabla^{\mathscr{V}_{M}}\right)$. Then there exists a normalizing function $\chi$ such that the bounded transform $\varphi(D)$ of $D$ associated with $\chi$ (i.e. $\left.\varphi=1-\chi^{2}\right)$ lies in the relative coarse $\mathrm{C}^{*}$-algebra of $(M, K)$ :

$$
\varphi(D)=I-\chi(D)^{2} \in \mathrm{C}_{K}^{*}(M ; A) .
$$

Proof. Let $\kappa_{0}>0$ be such that the scalar curvature $\kappa$ of the metric $g$ satisfies $\kappa \geq \kappa_{0}$ outside of $K$, and let $\chi$ be a normalizing function such that $\varphi=$ $1-\chi^{2}$ has support in $[-\alpha, \alpha]$ for $\alpha>0$ with $\alpha^{2}<\kappa_{0} / 4$. We will prove subsequently $\varphi(D) \in \mathrm{C}_{K}^{*}(M ; A)$ for such a choice of $\chi$. To this end we show that $\|\varphi(D) u\|$ becomes arbitrary small if the support of $u \in L^{2}(M, S)$ with $\|u\|=1$ is sufficiently far away from $K$, cf. Lemma 1.2.10. The argument will consist in deriving an estimate for $\|\varphi(D) u\|$ from more elementary estimates which contain $\|\varphi(D)\|$ as LHS and RHS, respectively, by means of a decomposition of $\varphi^{2}$ into functions whose growth we can control.

To begin with, set $f:=\varphi^{2}$ and let $\delta>0$. By Lemma 1.1.3 of the Appendix we can choose $f_{\delta}$ such that $f=f_{\delta}+r_{\delta}$ with a function $r_{\delta}$ such that $f_{\delta}=g_{\delta}^{2}$ for a function $g_{\delta} \in W$, and such that in addition

$$
\sup _{x \in \mathbb{R}}\left|x^{j} r_{\delta}(x)\right| \leq \delta
$$

for each $j=0,1,2$. Using these choices we now derive several estimates, which we will combine to obtain the desired result. For this let $u \in \Gamma_{\mathrm{cpt}}(M, S)$. Since $D$ is self-adjoint, one has

$$
\|\varphi(D) u\|^{2}=\langle f(D) u, u\rangle .
$$

Furthermore, our choice of $\varphi$ and $f_{\delta}$ together with the Cauchy-Schwarz inequality and basic properties of the functional calculus for $D$, imply the following two estimates (see Section 1.2 in the Appendix for more details)

$$
\left|\left\langle D^{2} f(D) u, u\right\rangle\right| \leq \alpha^{2}\|\varphi(D) u\|^{2}
$$

and

$$
\left|\left\langle D^{2} r_{\delta}(D) u, u\right\rangle\right| \leq \delta\|u\|^{2}
$$


Let $R>0$ be such that $\operatorname{supp}\left(\hat{g}_{\delta}\right) \subset[-R, R]$, then by a finite propagation speed argument, $\operatorname{supp}\left(g_{\delta}(D) u\right) \subset B(\operatorname{supp}(u) ; R)$. We have

$$
g_{\delta}(D)=\int_{-R}^{+R} \hat{g}_{\delta}(t) e^{i t D} d t=2 R \hat{g}_{\delta}\left(t_{0}\right) e^{i t_{0} D}
$$

for some $t_{0} \in(-R, R)$. Since $D$ has finite propagation speed $c_{D}=1$, one has $\operatorname{supp}\left(e^{i t_{0} D} u\right) \subset B\left(\operatorname{supp}(u) ;\left|t_{0}\right|\right)$ for each $u \in \Gamma_{\operatorname{cpt}}(M, S)$ and hence

$$
\begin{aligned}
\operatorname{supp}\left(g_{\delta}(D) u\right) & =\operatorname{supp}\left(e^{i t_{0} D} u\right) \\
& \subset B\left(\operatorname{supp}(u) ;\left|t_{0}\right|\right) \\
& \subset B(\operatorname{supp}(u) ; R) .
\end{aligned}
$$

Here we have used $\operatorname{supp}\left(g_{\delta}(D) u\right)=\operatorname{supp}\left(e^{i t_{0} D} u\right)$. This holds certainly if $\hat{g}_{\delta}\left(t_{0}\right)=0$ and otherwise because $\rho(\varphi) g_{\delta}(D) u=0$ is equivalent to the requirement that

$$
\begin{aligned}
0 & =\left\langle\rho(\varphi) g_{\delta}(D) u, v\right\rangle \\
& =\left\langle g_{\delta}(D) u, \rho(\varphi) v\right\rangle \\
& =\hat{g}_{\delta}\left(t_{0}\right)\left\langle e^{i t_{0} D} u, \rho(\varphi) v\right\rangle \\
& =\hat{g}_{\delta}\left(t_{0}\right)\left\langle\rho(\varphi) e^{i t_{0} D} u, v\right\rangle
\end{aligned}
$$

holds for each $v \in L^{2}(M, S)$, that is, to the requirement $\rho(\varphi) e^{i t_{0} D} u=0$.

To summarize, $\operatorname{supp}\left(g_{\delta}(D) u\right)$ is outside of $B(K ; R)$ if $u$ is such that $\operatorname{supp}(u) \cap$ $B(K ; 2 R)=\varnothing$. Hence $\operatorname{supp}\left(f_{\delta}(D) u\right)$ is outside of $B(K ; R)$ if the support of $u$ satisfies $\operatorname{supp}(u) \cap B(K ; 3 R)=\varnothing$.

From now on assume that $\operatorname{supp}(u)$ is outside of $B(K ; 3 R)$. Then, if $\Delta=\nabla^{*} \nabla$ is the connection Laplacian for the connection on the spinor bundle, we can derive using the Lichnerowicz formula, the following estimate

$$
\begin{aligned}
\left\langle D^{2} f_{\delta}(D) u, u\right\rangle & =\left\langle f_{\delta}(D) D^{2} u, u\right\rangle \\
& =\left\langle f_{\delta}(D)\left\{\Delta+\frac{\kappa}{4}\right\} u, u\right\rangle \\
& =\left\langle f_{\delta}(D)\left\{\Delta+\left(\frac{\kappa-\kappa_{0}}{4}\right)\right\} u, u\right\rangle+\frac{\kappa_{0}}{4}\left\langle f_{\delta}(D) u, u\right\rangle \\
& =\left\langle\left\{\Delta+\left(\frac{\kappa-\kappa_{0}}{4}\right)\right\} u, f_{\delta}(D) u\right\rangle+\frac{\kappa_{0}}{4}\left\langle f_{\delta}(D) u, u\right\rangle \\
& \geq \frac{\kappa_{0}}{4}\left\langle f_{\delta}(D) u, u\right\rangle
\end{aligned}
$$

for each $u \in \Gamma_{\mathrm{cpt}}(M, S)$ whose support lies outside of $B(K ; 3 R)$. Here we have used $f_{\delta}(D) \geq 0$ and that $\operatorname{supp}\left(f_{\delta}(D) u\right)$ is outside of $K$ and hence that $\kappa \geq \kappa_{0}$ holds there. Thus

$$
\left\langle D^{2} f_{\delta}(D) u, u\right\rangle \geq \frac{\kappa_{0}}{4}\left\langle f_{\delta}(D) u, u\right\rangle
$$


holds for each $u \in \Gamma_{\text {cpt }}(M, S)$ for which $\operatorname{supp}(u)$ lies outside of $B(K ; 3 R)$. Finally, we obtain using (1) - (4) the following estimate

$$
\begin{aligned}
\left(\frac{\kappa_{0}}{4}-\alpha^{2}\right)\|\varphi(D) u\|^{2} & \leq \frac{\kappa_{0}}{4}\langle f(D) u, u\rangle-\left\langle D^{2} f(D) u, u\right\rangle \\
& \leq \frac{\kappa_{0}}{4}\langle f(D) u, u\rangle-\left\langle D^{2} f_{\delta}(D) u, u\right\rangle-\left\langle D^{2} r_{\delta}(D) u, u\right\rangle \\
& \leq \frac{\kappa_{0}}{4}\langle f(D) u, u\rangle-\left\langle D^{2} f_{\delta}(D) u, u\right\rangle+\delta\|u\|^{2} \\
& \leq \frac{\kappa_{0}}{4}\langle f(D) u, u\rangle-\frac{\kappa_{0}}{4}\left\langle f_{\delta}(D) u, u\right\rangle+\delta\|u\|^{2} \\
& =\frac{\kappa_{0}}{4}\left\langle r_{\delta}(D) u, u\right\rangle+\delta\|u\|^{2} \\
& \leq\left(\frac{\kappa_{0}}{4}+1\right) \delta\|u\|^{2} .
\end{aligned}
$$

In conclusion, one has

$$
\|\varphi(D) u\|^{2} \leq\left(\frac{\kappa_{0}}{4}-\alpha^{2}\right)^{-1}\left(\frac{\kappa_{0}}{4}+1\right) \delta\|u\|^{2}
$$

for each $u \in \Gamma_{\mathrm{cpt}}(M, S)$ with $\operatorname{supp}(u)$ outside of $B(K ; 3 R)$.

Finally, let $\varepsilon>0$. Assume $u \in L^{2}(M, S)$ satisfies $\|u\|=1$ and that $\operatorname{supp}(u)$ lies outside of $B(K ; 3 R)$. Then there exists $v \in \Gamma_{\text {cpt }}(M, S)$ with $\|u-v\|<\varepsilon$ and $\operatorname{supp}(v)$ lying outside of $B(K ; 3 R)$. Then

$$
\begin{aligned}
\|\varphi(D) u\| & \leq\|\varphi(D)\| \varepsilon+\|\varphi(D) v\| \\
& \leq\|\varphi(D)\| \varepsilon+\left(\frac{\kappa_{0}}{4}-\alpha^{2}\right)^{-1}\left(\frac{\kappa_{0}}{4}+1\right) \delta\|v\|^{2} .
\end{aligned}
$$

The last expression becomes arbitrarily small if one chooses $\varepsilon$ and $\delta$ sufficiently small (and hence $R$ sufficiently large).

1.4.28 Theorem (Vanishing theorem). Let $(M, g)$ be as in Assumption 1.4.19. Assume that $K \subset M$ is a compact subset such that the scalar curvature of the metric is uniformly positive outside of $K$. Then $\operatorname{ind}_{p}(D) \in \mathrm{K}_{p}\left(\mathrm{C}^{*}(M, A)\right)$ vanishes for $p=0,1$. Here $D: \Gamma(M, S) \rightarrow \Gamma(M, S)$ with $S=\Sigma M \otimes \mathscr{V}_{M}$ is the twisted Dirac operator obtained by twisting the spinorial Dirac operator of $(M, g)$ with the Mishchenko line bundle $\left(\mathscr{V}_{M}, \nabla^{\mathscr{V}} M\right)$.

Proof. We first of all consider the case where $p=0$. For reasons of space we set $A=\mathrm{C}^{*} \pi_{1}(M)$ and abbreviate $\mathrm{C}^{*}(M ; A), \mathrm{D}^{*}(M ; A) \subset \mathscr{L}_{A}^{*}\left(L^{2}\left(M, S_{+}\right)\right)$ and $\mathscr{K}_{A}\left(L^{2}\left(M, S_{+}\right)\right)$as $\mathrm{C}^{*}, \mathrm{D}^{*}$ and $\mathscr{K}_{A}$. Using this notation we consider the following commutative diagram

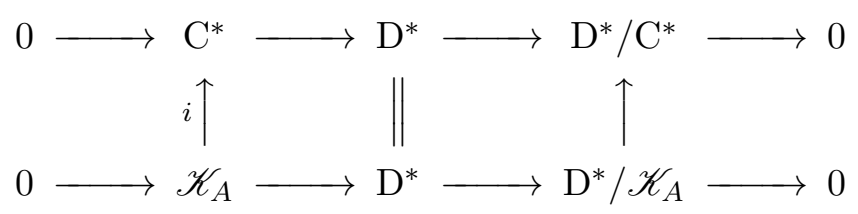




\section{Coarse index theory and positive scalar curvature}

in which $i$ denotes the inclusion map. From it we obtain by naturality of the six-term exact sequence the following commutative diagram

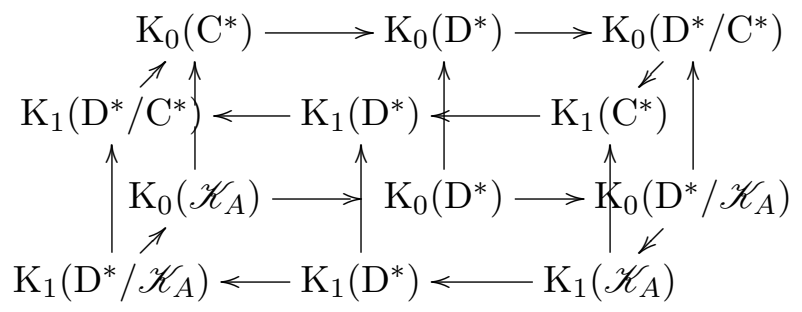

By Lemma 1.4.24 (and more restrictive assumptions like in Assumption 1.4.19) or Theorem 1.4.26 the map $\mathrm{K}_{0}(i)$ induced by the inclusion $i$ of $\mathscr{K}_{A}\left(L^{2}\left(M, S_{+}\right)\right)$ into $\mathrm{C}^{*}(M ; A)$ vanishes. According to Theorem 1.4.27 there exists a normalizing function $\chi$ such that $K:=I-\chi(D)^{2} \in \mathscr{K}_{A}\left(L^{2}(M, S)\right)$. Since

$$
\chi(D)=\left[\begin{array}{cc}
0 & \chi\left(D_{-}\right) \\
\chi\left(D_{+}\right) & 0
\end{array}\right]
$$

we thus find compact operators $K_{1} \in \mathscr{K}_{A}\left(L^{2}\left(M, S_{+}\right)\right)$and $K_{2} \in$ $\mathscr{K}_{A}\left(L^{2}\left(M, S_{-}\right)\right)$with

$$
\left[\begin{array}{ll}
I_{+}-\chi\left(D_{-}\right) \chi\left(D_{+}\right) & \\
& I_{-}-\chi\left(D_{+}\right) \chi\left(D_{-}\right)
\end{array}\right]=\left[\begin{array}{ll}
K_{1} & \\
& K_{2}
\end{array}\right]
$$

Here $I_{ \pm} \in \mathscr{L}_{A}^{*}\left(L^{2}\left(M, S_{ \pm}\right)\right)$is the identity operator. By definition $\operatorname{ind}_{0}(D)=$ $\partial_{1}\left[U^{*} \chi\left(D_{+}\right)\right]$. On the other hand $U^{*} \chi\left(D_{+}\right)$defines a unitary in $\mathrm{D}^{*} / \mathscr{K}_{A}$ as the following computations show:

$$
\begin{aligned}
\left(U^{*} \chi\left(D_{+}\right)\right)\left(\chi\left(D_{-}\right) U\right) & =U^{*} \chi\left(D_{+}\right) \chi\left(D_{-}\right) U \\
& =U^{*}\left(I_{-}-K_{2}\right)(D) U \\
& \sim I_{+} \bmod \mathscr{K}_{A} .
\end{aligned}
$$

and

$$
\begin{aligned}
\left(\chi\left(D_{-}\right) U\right)\left(U^{*} \chi\left(D_{+}\right)\right) & =\chi\left(D_{-}\right) \chi\left(D_{+}\right) \\
& =\left(I_{+}-K_{1}\right)(D) \\
& \sim I_{+} \bmod \mathscr{K}_{A} .
\end{aligned}
$$

This implies $\operatorname{ind}_{0}(D)=0$.

Finally, we can handle the case where $p=1$ analogously to the previous case. Again we set $A=\mathrm{C}^{*} \pi_{1}(M)$ and abbreviate $\mathrm{C}^{*}(M ; A), \mathrm{D}^{*}(M ; A) \subset$ $\mathscr{L}_{A}^{*}\left(L^{2}(M, S)\right)$ and $\mathscr{K}_{A}\left(L^{2}(M, S)\right)$ as $\mathrm{C}^{*}, \mathrm{D}^{*}$ and $\mathscr{K}_{A}$. Again there exists by naturality of the six-term exact sequence the following commutative diagram

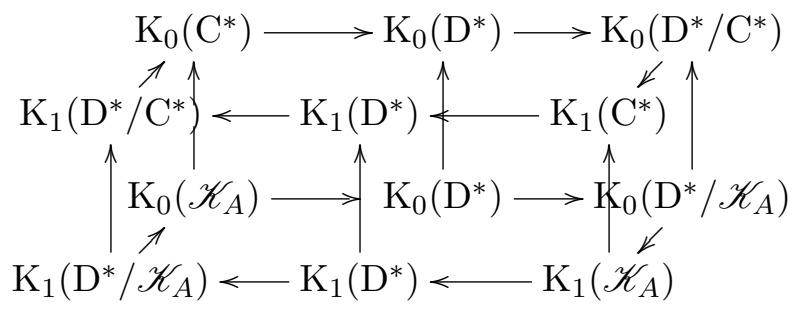


By Theorem 1.4.26 the map $\mathrm{K}_{1}(i)$ induced by the inclusion $i$ of $\mathscr{K}_{A}\left(L^{2}(M, S)\right)$ into $\mathrm{C}^{*}(M ; A)$ vanishes. Again there exists by Theorem 1.4 .27 a normalizing function $\chi$ such that $K:=I-\chi(D)^{2} \in \mathscr{K}_{A}$. By definition $\operatorname{ind}_{1}(D)=\partial_{1}[(I+$ $\chi(D)) / 2$ ] but the following computation shows that $(I+\chi(D)) / 2$ defines a projection in $\mathrm{D}^{*} / \mathscr{K}_{A}$ :

$$
\begin{aligned}
\left(\frac{I+\chi(D)}{2}\right)^{2} & =\left(\frac{I+\chi(D)}{2}\right)+\frac{\left(\chi^{2}-1\right)(D)}{4} \\
& \sim\left(\frac{I+\chi(D)}{2}\right) \bmod \mathscr{K}_{A} .
\end{aligned}
$$

This implies $\operatorname{ind}_{1}(D)=0$.

\subsection{Roe's partitioned manifold index theorem}

\subsubsection{Introduction}

The partitioned manifold index theorem of Roe relates the odd index of a Dirac operator on an odd dimensional complete Riemannian manifold, partitioned by a compact hypersurface, to the index of the Dirac operator on the hypersurface. The result appeared first 1989 in [35]. Two years after this, Higson gave a new proof of this theorem in [21]. On first sight Higson's formulation of the theorem differs from the one given by Roe, but actually they are equivalent as will be explained later in this section. Recently Zadeh [51] extended the theorem in Higson's formulation to the $\mathrm{C}^{*}$-linear context. A similar, though different, generalization in the same direction was given and applied before in [5] by Block and Weinberger.

\subsubsection{Dirac operators on hypersurfaces}

Before stating the partitioned manifold index theorem, we recall the definition of a partitioned manifold and that of an induced spin structure and then discuss Dirac operators on a hypersurfaces.

1.5.1 Definition (Partitioning hypersurface, partitioned manifold). Let $(M, g)$ be an oriented Riemannian manifold. A hypersurface in $M$ is an submanifold $N \subset M$ of codimension 1 (usually equipped with the induced metric) which is oriented by a unit normal vector field $\nu$. The hypersurface $N$ is a partitioning hypersurface if $M \backslash N$ is the disjoint union of two open (not necessarily connected) subsets $M_{+}$and $M_{-}$of $M$ with $\partial M_{ \pm}=N$. Where applicable, we say that $N$ partitions $M$ and that $M$ is a partitioned manifold with partitioning $\left(M, M_{+}, M_{-}\right)$. We will always orient $N$ such that the the unit normal vector field along $N$ points into $M_{+}$.

Of course, with this definition every manifold admits a partition (simply let $N$ be the boundary of a coordinate ball in $M$ ). A rather interesting question is 
whether a given manifold $N$ can be realized as a partitioning hypersurface in another given manifold.

5.2 Induced spin structure. A submanifold of a spin manifold needs not to be a spin manifold itself. Nevertheless, it is sometimes possible to induce a spin structure from an ambient manifold on a submanifold. Let $\left(M, g_{M}\right)$ be an $n$ dimensional Riemannian manifold with spin structure $\sigma_{M}=\left(\operatorname{Spin}(M), \Theta_{M}\right)$. We consider a $k$-codimensional submanifold $N \subset M$ of $M$ and ask whether and how we can use the given spin structure to induce a spin structure $\sigma_{N}$ on the manifold $N$.

Recall the following definition.

1.5.3 Definition (Reduction of a principal bundle/the structure group). Let $\lambda: H \rightarrow G$ be a continuous group homomorphism and $\left(P_{G}, \pi_{G}, M ; G\right)$ a $G$ principal bundle. A $H$-principal bundle $\left(P_{H}, \pi_{H}, M ; H\right)$ together with a continuous map $f: P_{H} \rightarrow P_{G}$ is a $\lambda$-reduction if the following diagram commutes:

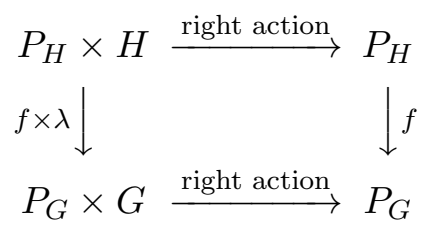

1.5.4 Definition (Induced spin structure). Let $(M, g)$ be a Riemannian spin manifold with spin structure $\left(\operatorname{Spin}(M), \Theta_{M}\right)$, and $N$ a hypersurface oriented by the normal vectorfield $\nu$. The sub-bundle $P$ of $\mathbf{S O}(M)_{\mid N}$ given by

$$
P:=\left\{\left(s_{1}, \ldots, s_{n-1}, \nu\right) ;\left(s_{1}, \ldots, s_{n-1}\right) \in \mathbf{S O}(N)\right\} \subset \mathbf{S O}(M)_{\mid N}
$$

is an $\mathrm{SO}(n-1)$-reduction of $\mathbf{S O}(M)_{\mid N}$ which is isomorphic to $\mathbf{S O}(N)$ as a $\mathrm{SO}(n-1)$-principal bundle. In this case $f$ is the inclusion map, the action of $\mathrm{SO}(n-1)$ on $P$ is the one induced by the inclusion $\mathrm{SO}(n-1) \subset \mathrm{SO}(n)$ and the given action of $\operatorname{SO}(n)$ on $\mathbf{S O}(M)$. Finally, let $\sigma_{N}=\left(\operatorname{Spin}(N), \Theta_{N}\right)$ be the spin structure given by $\operatorname{Spin}(N):=\Theta_{M}^{-1}(P) \subset \operatorname{Spin}(M)_{\mid N}$ with the action of $\operatorname{Spin}(n-1)$ induced by the $\operatorname{Spin}(n)$-action on $\operatorname{Spin}(M)_{\mid N}$, and $\Theta_{N}:=$ $\left(\Theta_{M}\right)_{\mid \operatorname{Spin}(N)}$ as bundle projection. That this defines indeed a spin structure follows directly from the fact that $\sigma_{M}$ is such a structure. The spin structure $\sigma_{N}$ is called the induced spin structure.

1.5.5 Example. Let $M$ be a oriented $(n+1)$-dimensional manifold for which $\mathbf{S O}(M)$ is trivial and which is equipped with the trivial spin structure, and let $N \subset M$ be a 1-codimensional submanifold oriented by the outward pointing normal vector field $\nu$. By choosing a trivializing global oriented orthonormal frame $\left(E_{1}, \ldots, E_{n+1}\right)$, we can identify $\mathbf{S O}(M)$ with $M \times \operatorname{SO}(n+1)$. Simply identify the frame $\left(E_{1}(p), \ldots, E_{n+1}(p)\right) \cdot A$, with $A \in \mathrm{SO}(n+1)$. for a given point $p$ with $(p, A) \in M \times \mathrm{SO}(n+1)$. The bundle $\mathrm{SO}(M)_{\mid N}$ is also trivial. Let us also assume that there exists a global section $\left(E_{1}, \ldots, E_{n}\right)$ of $\mathbf{S O}(N)$ and consider the global section $\left(E_{1}, \ldots, E_{n}, \nu\right)$ with last component equal to $\nu$. 
This identifies $\mathbf{S O}(M)_{\mid N}$ with $N \times \mathrm{SO}(n+1)$, and $P$ with $N \times \mathrm{SO}(n)$. Since $\Theta_{M}=\operatorname{id}_{M} \times \theta_{n+1}$, we have

$$
\operatorname{Spin}(N)=\Theta_{M}^{-1}(P)=\left\{(p, g) \in N \times \operatorname{Spin}(n+1) ; \theta_{n+1}(g) \in \operatorname{Spin}(n)\right\} .
$$

The diagram

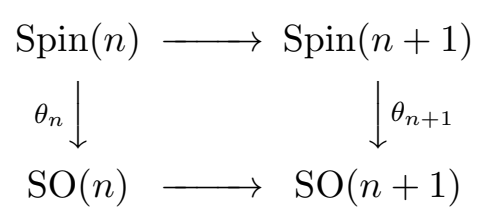

commutes and $\theta_{n+1}(g) \in \mathrm{SO}(n)$ is equivalent to $g \in \operatorname{Spin}(n)$, we thus find that $\Theta_{M}^{-1}(P)=N \times \operatorname{Spin}(n)$ is the trivial spin structure on $N$.

5.6 Dirac operators on hypersurfaces. Here we recall some well-known results on the Dirac operator on a hypersurface. See [2] and [14]. For this purpose let $\left(M, g_{M}\right)$ be an $(n+1)$-dimensional Riemannian manifold with metric $g_{M}$ and spin structure $\sigma_{M}=\left(\operatorname{Spin}(M), \Theta_{M}\right)$. The associated Dirac operator $D_{M}: \Gamma(M, \Sigma M) \rightarrow \Gamma(M, \Sigma M)$ acts on sections of the associated spinor bundle $\Sigma M=\operatorname{Spin}(M) \times{ }^{\sigma_{2 n+1}} \Delta_{2 n+1}$. We consider a hypersurface $N$ of $M$ which is oriented by a normal vector field $\nu$, and we equip this submanifold with the induced metric $g_{N}=i^{*} g_{M}$ (here $i: N \rightarrow M$ is the inclusion map) and the induced spin structure $\left(\operatorname{Spin}(N), \Theta_{N}\right)$. We will describe the intrinsically defined Dirac operator $D_{N}: \Gamma(N, \Sigma N) \rightarrow \Gamma(N, \Sigma N)$ extrinsically by means of the spinor bundle $\Sigma M$, the Clifford multiplication $c_{M}$, and the covariant spinor derivative $\nabla^{\Sigma M}$ induced by the covariant Levi-Civita derivative $\nabla^{M}$ of the ambient manifold $M$. As the following table makes clear, the rank of $\Sigma M$ can only be equal to that of $\Sigma N$ if $n=\operatorname{dim}(N)$ is even (let $k \in \mathbb{N}_{0}$ and $\langle n\rangle$ the dimension of the representation space $\Delta_{n}$ ).

\begin{tabular}{|c|c|c|}
\hline & $n=2 k$ & $n=2 k+1$ \\
\hline$\langle n\rangle=\left\lfloor\frac{n}{2}\right\rfloor$ & $k$ & $k$ \\
\hline$\langle n+1\rangle=\left\lfloor\frac{n}{2}\right\rfloor$ & $k$ & $k+1$ \\
\hline
\end{tabular}

From now on we assume $n=\operatorname{dim}(N)$ to be even.

1.5.7 Lemma. Let $n=2 k$ be even and let $\left(e_{1}, \ldots, e_{n}, e_{n+1}\right)$ be an orthonormal basis of $\mathbb{R}^{n+1}$ (with the usual Euclidean scalar product). Then the algebra isomorphism $\alpha: \mathrm{Cl}^{c}(n) \rightarrow \mathrm{Cl}^{c}(n+1)^{0}$ with $\alpha\left(e_{j}\right)=e_{j} e_{n+1}$ (for $j=1, \ldots, n)$ restricts on $\operatorname{Spin}(n)$ to the inclusion map from $\operatorname{Spin}(n)$ into $\operatorname{Spin}(n+1)$. If $\kappa_{n+1}: \mathrm{Cl}^{c}(n+1) \rightarrow \operatorname{End}\left(\Delta_{n+1}\right)$ is an irreducible algebra representation, then $\kappa_{n}:=\kappa_{n+1} \circ \alpha$ defines an irreducible algebra representation $\kappa_{n}: \mathrm{Cl}^{c}(n) \rightarrow \operatorname{End}\left(\Delta_{n}\right)$ (set $\left.\Delta_{n}:=\Delta_{n+1}\right)$. Hence these representations are up to equivalence the unique spinor representations of the Clifford algebras $\mathrm{Cl}^{c}(n)$ and $\mathrm{Cl}^{c}(n+1)$. Furthermore, $\Delta_{n}^{ \pm}=\operatorname{Eig}\left(M_{e_{n+1}} ; \pm \sqrt{-1}\right) \subset \Delta_{n+1}$ is the eigenspace of the endomorphism of $\Delta_{n+1}$, which is given by multiplication with $e_{n+1}$ from the right.

1.5.8 Definition (Restriction of a Dirac operator). Let $M$ and $N$ be as in Section 1.5.2. We drop the spin condition and assume only that $\left(S, \nabla^{S}\right)$ is a 
Dirac bundle on $M$ (for instance, the spinorial Dirac bundle coming from a spin structure on $M)$ with associated Dirac operator $D: \Gamma(M, S) \rightarrow \Gamma(M, S)$. In this situation, the restriction of $\left(S, \nabla^{S}\right)$ to $N$, written $\operatorname{res}_{N}\left(S, \nabla^{S}\right)$, is the Dirac bundle $\left(T, \nabla^{T}\right)$ given by the following data: the Dirac bundle $T:=S_{\mid N}$, the restriction of $S$ to $N$ equipped with the Clifford multiplication $\bar{c}_{N}: T N \rightarrow$ $\operatorname{End}\left(S_{\mid N}\right)$ with $\bar{c}_{N}(v)\left(s_{x}\right)=c_{M}(v)\left(s_{x}\right)$, where $c_{M}: T M \rightarrow \operatorname{End}(S)$ is Clifford multiplication for $S$. The covariant derivative operator $\nabla^{T}$ is ${ }^{12}$

$$
\nabla_{X}^{T}\left(s_{\mid N}\right):=\left(\nabla_{X}^{S} s+\frac{1}{2}\left(\nabla_{X}^{M} \nu\right) \cdot \nu \cdot s\right)_{\mid N} .
$$

Here $X$ is a vector field tangent to $N$ and $s \in \Gamma(M, S)$ is a section with restriction $s_{\mid N} \in \Gamma(N, T)$ and $\nabla^{M}$ is the usual covariant derivative operator of the Levi-Civita connection given by the metric of $M$. The restriction of $D$ to the hypersurface $N$ is the Dirac operator

$$
\operatorname{res}_{N}(D): \Gamma\left(N, S_{\mid N}\right) \rightarrow \Gamma\left(N, S_{\mid N}\right)
$$

associated with the restricted Dirac bundle of $\left(S, \nabla^{S}\right)$. On $\operatorname{res}_{N}\left(S, \nabla^{S}\right)$ we consider the grading operator $\gamma_{\operatorname{res}_{N}\left(S, \nabla^{S}\right)}: S_{\mid N} \rightarrow S_{\mid N}$ with $\gamma_{\operatorname{res}_{N}}\left(s_{x}\right):=$ $c_{N}(\nu(x))\left(s_{x}\right)$ for $s_{x} \in S_{x}$ over $x \in N$.

We can now state the desired description of the instrinsic Dirac operator by exterior data.

1.5.9 Proposition. With the notation from Subsection 1.5.2, there exists an isomorphism between $\operatorname{res}_{N}\left(\Sigma M, \nabla^{\Sigma M}\right)$ and the intrinsically defined spinorial Dirac bundle $\left(\Sigma N, \nabla^{\Sigma N}\right)$ which respects the $\mathbb{Z} / 2$-grading (recall that $n$ is even).

Proof. To begin with, recall $\Delta_{2 n}=\Delta_{2 n+1}=\mathbb{C}^{\langle n\rangle}$ as vector spaces. We explain how one can realize the spin representaions $\sigma_{2 n}$ and $\sigma_{2 n+1}$ in such a way that $\sigma_{2 n+1 \mid \operatorname{Spin}(2 n)}=\sigma_{2 n}$. After this is done, a vector bundle isomorphism $\Phi: \Sigma N \rightarrow$ $(\Sigma M)_{\mid N}$ can be obtained from the following readily verified result: if $f: P_{H} \rightarrow$ $P_{G}$ is a reduction for $\lambda: H \rightarrow G$, and if $\rho_{G}: G \rightarrow \operatorname{GL}(V)$ and $\rho_{H}: H \rightarrow \operatorname{GL}(V)$ are finite-dimensional linear group representations with $\rho_{H}=\rho_{G} \circ \lambda$, then the map $\Phi: P_{H} \times{ }^{\rho_{H}} V \rightarrow P_{G} \times{ }^{\rho_{G}} V$ with $\Phi[p, v]=[f(p), v]$ is an injective homomorphism of vector bundles of the same rank, and thus an isomorphism. In our case $f: \operatorname{Spin}(N)=\Theta^{-1}(P) \rightarrow \operatorname{Spin}(M)_{\mid N}$ is the inclusion map and $\lambda: \operatorname{Spin}(n-1) \rightarrow \operatorname{Spin}(n)$ is the inclusion obtained from $\mathbb{R}^{n-1} \subset \mathbb{R}^{n}$. Thus $\Phi: \Sigma N \rightarrow(\Sigma M)_{\mid N}$ is given by $\Phi[p, v]=[p, v]$.

In order to be able to see that $\Phi$ is compatible with the $\mathbb{Z} / 2$-gradings, i.e. $\Phi \circ \gamma_{\Sigma N}=\gamma_{(\Sigma M)_{\mid N}} \circ \Phi$, we recall how the Clifford multiplication $c_{M}: \mathfrak{X}(M) \times \Gamma(M, \Sigma) \rightarrow \Gamma(M, \Sigma M)$ is defined: first of all, one identifies $T M$ with $\operatorname{Spin}(M) \times^{\rho} \mathbb{R}^{2 n+1}$, where $\rho: \operatorname{Spin}(2 n+1) \rightarrow \mathrm{GL}\left(\mathbb{R}^{n}\right)$ is the linear representation given by the left regular representation of $\mathrm{SO}(2 n+1)$ on $\mathbb{R}^{2 n+1}$ and

\footnotetext{
${ }^{12}$ The second summand in this definition - the first one is given by the pullback derivative operator - has to be added to guarantee the compatibility with the Levi-Civita connection on $N$.
} 
the spin covering $\Theta_{2 n+1}: \operatorname{Spin}(2 n+1) \rightarrow \mathrm{SO}(2 n+1)$. Under this identification the Clifford multiplication $\cdot: \mathbb{R}^{2 n+1} \otimes \Delta_{2 n+1} \rightarrow \Delta_{2 n+1}$ induces a multiplication between tangent vectors and spinors via (let $x \in M$ )

$$
T_{x} M \otimes(\Sigma M)_{x} \rightarrow(\Sigma M)_{x} \quad, \quad[p, v] \otimes[p, \psi] \mapsto[p, v \cdot \psi] .
$$

If the identification of $T M$ with $\operatorname{Spin}(M) \times^{\rho} \mathbb{R}^{2 n+1}$ is given by the obvious map $T M \rightarrow \operatorname{Spin}(M) \times^{\rho} \mathbb{R}^{2 n+1}$, which maps as follows:

$$
\sum_{i=1}^{2 n+1} \lambda_{i} E_{i}(x) \mapsto\left[\Theta_{M}^{-1}\left(E_{1}, \ldots, E_{2 n+1}\right),\left(\lambda_{1}, \ldots, \lambda_{2 n+1}\right)\right],
$$

then $\nu(x)$ corresponds to $\left[\Theta_{M}^{-1}\left(E_{1}, \ldots, E_{2 n}, \nu\right), e_{2 n+1}\right]$. Thus, the Clifford multiplication by $\nu(x)$ is given by the Clifford multiplication by $e_{2 n+1}$ on $\Delta_{2 n+1}$. With this at hand one obtains

$$
\begin{aligned}
\Phi\left(\gamma_{\Sigma N}\left(\psi_{x}\right)\right) & =\Phi\left(\nu(x) \cdot \psi_{x}\right) \\
& =\Phi\left[\Theta_{M}^{-1}\left(E_{1}, \ldots, E_{2 n}, \nu\right), e_{n+1} \cdot v\right] \\
& =\gamma_{\Sigma M}\left(\psi_{x}\right)
\end{aligned}
$$

for any $\psi_{x}=\left[\Theta_{M}^{-1}\left(E_{1}, \ldots, E_{2 n}, \nu\right), v\right]$ in $\Sigma N$.

Now Lemma 1.5.7 shows how one has to choose the spin representations.

In order to see that $\Phi \circ\left(\nabla_{X}^{\Sigma N} s\right)=\nabla_{X}^{\operatorname{res}_{N}(\Sigma M)}(\Phi \circ s)$ holds for any section $s \in \Gamma(N, \Sigma N)$ and each $X \in \mathfrak{X}(N)$ we make the following remarks: by definition of $\nabla^{\Sigma N}$ one has

$$
\nabla_{X}^{\Sigma N} s=X . s+\frac{1}{2} \sum_{1 \leq i<j \leq n} g_{N}\left(\nabla_{X}^{N} E_{i}, E_{j}\right) c_{N}\left(E_{i}\right)\left(c_{N}\left(E_{j}\right) . s\right)
$$

for all $X \in \mathfrak{X}(N), s \in \Gamma(N, \Sigma N)$ and any $\mathbf{S O}(N)$-frame $\left(E_{1}, \ldots, E_{n}\right)$. Analogously, one has

$$
\nabla_{Y}^{\Sigma M} t=Y . t+\frac{1}{2} \sum_{1 \leq k<l \leq n+1} g_{M}\left(\nabla_{Y}^{M} F_{k}, F_{l}\right) c_{M}\left(F_{k}\right)\left(c_{M}\left(F_{l}\right) \cdot t\right)
$$

for all $Y \in \mathfrak{X}(M), t \in \Gamma(M, \Sigma M)$ and any $\mathbf{S O}(M)$-frame $\left(F_{1}, \ldots, F_{n}, F_{n+1}\right)$. Now let $\nu: N \rightarrow T M_{\mid N}$ be the given unit normal field and $\left(E_{1}, \ldots, E_{n}\right)$ an $\mathbf{S O}(N)$-frame. Then for each $x \in N$ the tuple $\left(E_{1}(x), \ldots, E_{n}(x), \nu(x)\right)$ is an oriented orthonormal basis for $T_{x} N$ and we can do the following calculation valid on $N$ :

$$
\begin{aligned}
\nabla_{Y}^{\Sigma_{M} M} t=Y . & +\frac{1}{2} \sum_{1 \leq k<l \leq n} g_{M}\left(\nabla_{Y}^{M} E_{k}, E_{l}\right) c_{M}\left(E_{k}\right)\left(c_{M}\left(E_{l}\right) \cdot t\right) \\
& +\frac{1}{2} \sum_{k=1}^{n} g_{M}\left(\nabla_{Y}^{M} E_{k}, \nu\right) c_{M}\left(E_{k}\right)\left(c_{M}(\nu) \cdot t\right) \\
=Y . & +\frac{1}{2} \sum_{1 \leq k<l \leq n} g_{M}\left(\nabla_{Y}^{M} E_{k}, E_{l}\right) c_{M}\left(E_{k}\right)\left(c_{M}\left(E_{l}\right) \cdot t\right) \\
& +\frac{1}{2} c_{M}\left(\sum_{k=1}^{n} g_{M}\left(\nabla_{Y}^{M} E_{k}, \nu\right) E_{k}\right)\left(c_{M}(\nu) \cdot t\right) .
\end{aligned}
$$


Since one has

$$
g_{M}\left(\nabla_{Y}^{M} E_{k}, \nu\right)=-g_{M}\left(E_{k}, \nabla_{Y}^{M} \nu\right)+Y . g_{M}\left(E_{k}, \nu\right)=-g_{M}\left(E_{k}, \nabla_{Y}^{M} \nu\right)
$$

for any $1 \leq k \leq n$, we can rewrite the sum occuring in the third summand term of Equation (1.5.4) in the following way:

$$
\begin{aligned}
\sum_{k=1}^{n} g_{M}\left(\nabla_{Y}^{M} E_{k}, \nu\right) E_{k} & =-\sum_{k=1}^{n} g_{M}\left(\nabla_{Y}^{M} \nu, E_{k}\right) E_{k} \\
& =g_{M}\left(\nabla_{Y}^{M} \nu, \nu\right) \nu-\nabla_{Y}^{M} \nu \\
& =-\nabla_{Y}^{N} \nu .
\end{aligned}
$$

Here the last equality comes from the definition of $\nabla^{N}$. Now notice that $g_{M}\left(\nabla_{Y}^{M} E_{k}, E_{l}\right)=g_{N}\left(\nabla_{Y}^{N} E_{k}, E_{l}\right)$ holds by the following computation

$$
\begin{aligned}
g_{M}\left(\nabla_{Y}^{M} E_{k}, E_{l}\right) & =g_{M}\left(\nabla_{Y}^{N} E_{k}+g_{M}\left(\nabla_{Y}^{M} E_{k}, \nu\right) \nu, E_{l}\right) \\
& =g_{M}\left(\nabla_{Y}^{N} E_{k}, E_{l}\right)+g_{M}\left(\nabla_{Y}^{M} E_{k}, \nu\right) g_{M}\left(\nu, E_{l}\right) \\
& =g_{M}\left(\nabla_{Y}^{N} E_{k}, E_{l}\right) \\
& =g_{N}\left(\nabla_{Y}^{N} E_{k}, E_{l}\right) .
\end{aligned}
$$

Thus we finally obtain from (1.5.4) and (1.5.5) the following formula:

$$
\begin{aligned}
\nabla_{Y}^{\Sigma M} t=Y . t & +\frac{1}{2} \sum_{1 \leq k<l \leq n} g_{N}\left(\nabla_{Y}^{M} E_{k}, E_{l}\right) c_{M}\left(E_{k}\right)\left(c_{M}\left(E_{l}\right) . t\right) \\
& -\frac{1}{2} c_{M}\left(\nabla_{Y}^{N} \nu\right)\left(c_{M}(\nu) . t\right) .
\end{aligned}
$$

Setting $t=\Phi \circ s$ and $Y=X$ we obtain from equation (1.5.1) and (1.5.6) the following formula, valid on $N$ :

$$
\begin{gathered}
\left(\nabla_{X}^{\mathrm{res}_{N}(\Sigma M)}(\Phi \circ s)\right)(x)=\nabla_{X}^{\Sigma M}(\Phi \circ s)+\frac{1}{2} c_{M}\left(\nabla_{X}^{M} \nu\right)\left(c_{M}(\nu)(\Phi \circ s)\right) \\
=X .(\Phi \circ s)+\frac{1}{2} \sum_{1 \leq k<l \leq n} g_{N}\left(\nabla_{Y}^{M} E_{k}, E_{l}\right) \therefore \\
\\
\because c_{M}\left(E_{k}\right)\left(c_{M}\left(E_{l}\right)(\Phi \circ s)\right) .
\end{gathered}
$$

One has $c_{M}\left(E_{k}\right)\left(c_{M}\left(E_{l}\right)(\Phi \circ s)\right)=c_{N}\left(E_{k}\right)\left(c_{N}\left(E_{l}\right)(\Phi \circ s)\right)$ for all $1 \leq k, l \leq n$ as follows from the following two computations: let $v \in T_{x} N \subset T_{x} M$, Then $v=\left(v^{1}, \ldots, v^{2 n}, 0\right)$ with respect to the basis $\left(E_{1}(x), \ldots, E_{2 n}(x), \nu(x)\right)$. Let $f_{s}: \operatorname{Spin}(N) \rightarrow \Delta_{2 n}$ be an equivariant function with $s(x)=\left[\tilde{x}, f_{s}(\tilde{x})\right]$ for any $x \in N$ covered by $\tilde{x} \in \mathbf{S p i n}(N)$. With this notation, one has on one hand

$$
\begin{aligned}
c_{M}(v)(\Phi(s(x))) & =c_{M}(v)\left[\tilde{x}, f_{s}(\tilde{x})\right] \\
& =\left[\tilde{x}, \kappa_{2 n+1}\left(v^{1}, \ldots, v^{2 n}, 0\right)\left(f_{s}(\tilde{x})\right)\right]
\end{aligned}
$$

and on the other hand

$$
\begin{aligned}
\Phi\left(c_{N}(v)(s(x))\right) & =\left[\tilde{x}, \kappa_{2 n}\left(v^{i} e_{i}\right)\left(f_{s}(\tilde{x})\right)\right] \\
& =\left[\tilde{x}, \kappa_{2 n+1}\left(v^{i} e_{i} e_{n+1}\right)\left(f_{s}(\tilde{x})\right)\right] \\
& =\left[\tilde{x}, \kappa_{2 n+1}\left(v^{i} e_{i}\right)\left(\kappa_{2 n+1}\left(e_{n+1}\right)\left(f_{s}(\tilde{x})\right)\right)\right] \\
& =\left[\tilde{x},-\kappa_{2 n+1}\left(e_{n+1}\right)\left(\kappa_{2 n+1}\left(v^{i} e_{i}\right)\left(f_{s}(\tilde{x})\right)\right)\right] .
\end{aligned}
$$


Notice that $e_{2 n+1}^{2}=-1$. Thus the claim follows by comparing equation (1.5.2) with equation (1.5.7).

\subsubsection{The theorem}

Let $M$ be a complete Riemannian spin manifold of odd dimension, which is partitioned by a compact hypersurface. In [35] Roe constructs a homomorphism $\zeta_{N}: \mathrm{K}_{1}\left(\mathrm{C}^{*}(M)\right) \rightarrow \mathbb{Z}$ using the pairing between odd K-theory and odd cyclic cohomology. For the moment we postpone the definition of this homomorphism and state the partitioned manifold index theorem. After this we will describe how the homomorphism is constructed.

1.5.10 Theorem (Partitioned manifold index theorem). Let $(M, g)$ be a complete Riemannian spin manifold of odd dimension. Assume that $M$ is partitioned by a compact hypersurface $N \subset M$. Then the equality

$$
\zeta_{N}\left(\operatorname{ind}_{1}\left(D_{M}\right)\right)=\operatorname{ind}\left(D_{N}\right)
$$

holds.

We continue with the construction of Roe's homomorphism $\zeta_{N}$. This is constructed using Connes' cyclic cohomology whose definition we recall next.

1.5.11 Definition. For each complex algebra $A$ one defines the Hochschild cochain complex $\left(C^{k}(A), b_{k}\right)_{k \in \mathbb{N}_{0}}$ and the subcomplex $\left(C_{\lambda}^{k}(A), b_{k}\right)_{k \in \mathbb{N}_{0}}$ given by the cyclic cochain complex by the following data:

1. A cochain (of degree $k$ ) is a $\left(k+1\right.$ )-linear functional $\phi: A^{k+1} \rightarrow \mathbb{C}$. The (additive) group of all cochains of degree $k$ is denoted by $C^{k}(A)$. It is customary to denote the elements of $A^{k+1}$ by $\left(a_{0}, \ldots, a_{k}\right)$.

2. A cyclic cochain (of degree $k$ ) is a chain $\phi \in C^{k}(A)$ which satisfies the following cyclic permutation condition: One has

$$
\phi\left(a_{0}, \ldots, a_{k}\right)=(-1)^{k} \phi\left(a_{k}, a_{0}, \ldots, a_{k-1}\right) .
$$

If one defines the cyclic permutation operator

$$
\lambda=\lambda_{k}: C^{k}(A) \rightarrow C^{k}(A)
$$

by

$$
\left(\lambda_{k} \phi\right)\left(a_{0}, \ldots, a_{k}\right):=(-1)^{k} \phi\left(a_{k}, a_{0}, \ldots, a_{k-1}\right)
$$

then the (additive) group of all cyclic cochains of degree $k$, denoted by $C_{\lambda}^{k}(A)$, is exactly $\operatorname{ker}\left(1-\lambda_{k}\right)$.

3. The boundary map

$$
b=b_{k}: C^{k}(A) \rightarrow C^{k+1}(A)
$$

as well as the induced boundary map (denoted by the same letter)

$$
b=b_{k}: C_{\lambda}^{k}(A) \rightarrow C_{\lambda}^{k+1}(A)
$$


is given by the formula

$$
\begin{array}{r}
\left(b_{k} \phi\right)\left(a_{0}, \ldots, a_{k+1}\right):=\sum_{j=0}^{k}(-1)^{j} \phi\left(a_{0}, \ldots, a_{j} a_{j+1}, \ldots, a_{k+1}\right) \\
+(-1)^{k} \phi\left(a_{k+1} a_{0}, a_{1}, \ldots, a_{k}\right)
\end{array}
$$

for $\phi \in C^{k}(A)$ and $\phi \in C_{\lambda}^{k}(A)$.

Finally, the Hochschild cohomology (of degree $k$ ) of $A$, denoted by $H^{k}(A)$ and the cyclic cohomology (of degree $k$ ) of $A$, denoted by $H_{\lambda}^{k}(A)$, is defined as the homology (of degree $k$ ) of the Hochschild and cyclic cochain complex, respectively.

Let the complex algebra in the previous definition be the Roe $\mathrm{C}^{*}$-algebra $\mathrm{C}^{*}(M)$. In [35] Roe considers the cyclic cocycle $\zeta \in C_{\lambda}^{1}\left(\mathrm{C}^{*}(M)\right)$ given by

$$
\zeta:(A, B) \mapsto \operatorname{Tr}([P A P, P B P]-P[A, B] P) .
$$

Here $P$ is the operator given by multiplication by the characteristic function of $M_{+}$. This defines a class in the odd cyclic cohomology $H_{\lambda}^{\text {odd }}\left(\mathrm{C}^{*}(M)\right)$ of the Roe $\mathrm{C}^{*}$-algebra. Recall that there exists for any $\mathrm{C}^{*}$-algebra $A$ a pairing

$$
\langle\cdot, \cdot\rangle: \mathrm{K}_{1}(A) \times H_{\lambda}^{\text {odd }}(A) \rightarrow \mathbb{C}
$$

between the odd $\mathrm{K}$-theory and odd cyclic cohomology of $A$. It is given by the following formula for which we refer the reader to [26, p. 157]: for $[u] \in \mathrm{K}_{1}(A)$ represented by $u \in \mathrm{GL}_{\infty}(A)$ and $[\phi] \in H_{\lambda}^{2 n-1}(A)$ represented by $\phi \in C_{\lambda}^{2 n-1}(A)$ one defines

$$
\langle[u],[\phi]\rangle=C_{n} \phi\left(u^{-1}-1, u-1, \ldots, u^{-1}-1, u-1\right)
$$

where $C_{n} \in \mathbb{R}$ is a constant depending only on $n \in \mathbb{N}$. With this notation, Roe's homomorphism is given as follows

$$
\zeta_{N}: \mathrm{K}_{1}\left(\mathrm{C}^{*}(M)\right) \rightarrow \mathbb{Z} \quad, \quad[u] \mapsto\langle[u],[\zeta]\rangle .
$$

As we have already remarked in the introduction to this section, there is a second version of the partitioned manifold index theorem due to Higson. We set out to briefly review Higson's approach and then relate this version with the cyclic cocycle-description of Roe. Higson (and following him, also Zadeh) consider the Cayley transform

$$
U:=(D+i I)(D-i I)^{-1}
$$

of $D$, and the operator

$$
U_{+}=M_{\phi_{-}}+M_{\phi_{+}} U
$$

obtained from it. Here $\phi_{+}$is a smooth function on $M$ which coincides outside a compact neighbourhood of $N$ with the characteristic function of $M_{+}$, and $\phi_{-}$ is defined as $\phi_{-}:=1-\phi_{+}$. As it turns out, this operator is Fredholm (in the case of Zadeh's paper it is Fredholm in the sense of Fomenko and Mishchenko) and its Fredholm index equals the index of $D_{N}$. This is Higson's formulation of Roe's index theorem for partitioned manifolds which we now state as a theorem. 


\section{Coarse index theory and positive scalar curvature}

1.5.12 Theorem (Partitioned manifold index theorem). Let $(M, g)$ be a complete Riemannian spin manifold of odd dimension. Assume that $M$ is partitioned by a compact hypersurface $N \subset M$. Then the equality

$$
\operatorname{ind}\left(U_{+}\right)=\operatorname{ind}\left(D_{N}\right)
$$

holds.

We now try to explain why this formulation is equivalent to the original one. For this we follow a corresponding remark in [18, p. 44]. One has $U=\varphi(D)$ if the normalizing function is chosen as $\chi(t)=\frac{2}{\pi} \arctan (t)$ and by definition $\operatorname{ind}_{1}(D)$ is the class $[U] \in \mathrm{K}_{1}\left(\mathrm{C}^{*}(M)\right)$. Recall, that there exists the following index pairing between the $\mathrm{K}$-homology and K-theory of a $\mathrm{C}^{*}$-algebra $A$

$$
\begin{aligned}
\langle\cdot, \cdot\rangle: & K^{1}(A) \times K_{1}(A) \\
([\mathscr{H}, \rho, F],[u]) & \rightarrow
\end{aligned} \quad \operatorname{ind}(P u P-(1-P): P \mathscr{H} \rightarrow P \mathscr{H})
$$

where $P:=(1+F) / 2$. If $A=\mathrm{C}^{*}(M)$ is the Roe $\mathrm{C}^{*}$-algebra, we can consider the cycle

$$
\left[L^{2}(M, S), \rho_{\text {std }}, 2 P-1\right]
$$

with $P:=M_{\chi_{+}}$, where $\chi_{+}$is the charactistic function of the $M_{+}$. The index pairing of this cycle with the odd index class $\operatorname{ind}_{1}(D)=[U]$ is exactly the index $\operatorname{ind}\left(U_{+}\right)$considered by Higson. The relation between the pairing $(*)$ between cyclic cohomology and K-homology and the pairing (**) between K-theory and K-homology is given by Connes' Chern character formula (also known as Connes' index theorem)

$$
\left\langle\mathrm{Ch}^{\text {odd }}(\mathscr{H}, F),[u]\right\rangle=\langle(\mathscr{H}, \rho, F),[u]\rangle,
$$

which can be found for example in [26, Prop. 4.2.5, p. 176]. Using this, the equivalence of the two stated formulations of the partitioned manifold index 
1. Coarse index theory and positive scalar curvature

theorem follows from the subsequent computation:

$$
\begin{aligned}
\zeta_{N}[u] & =\langle[u],[\zeta]\rangle \\
& =\frac{1}{4} \zeta\left(u^{-1}-1, u-1\right) \\
& =\frac{1}{4} \operatorname{Tr}\left(\left[P\left(u^{-1}-1\right) P, P(u-1) P\right]-P\left[u^{-1}-1, u-1\right] P\right) \\
& =\frac{1}{4} \operatorname{Tr}\left(P u^{-1} P u P-P u P u^{-1} P\right) \\
& =\operatorname{Tr}\left(P-\left(P u^{-1} P u P\right)\right)-\operatorname{Tr}\left(P-\left(P u P u^{-1} P\right)\right) \\
& =\operatorname{Tr}\left(P-\left(P u^{-1} P u P\right)\right)-\operatorname{Tr}\left(\left(P u 1 u^{-1} P\right)-\left(P u P u^{-1} P\right)\right) \\
& =\operatorname{Tr}\left(P-\left(P u^{-1} P u P\right)\right)-\operatorname{Tr}\left(P u(1-P)(P u(1-P))^{*}\right) \\
& =-\operatorname{Tr}\left(\left(-P+P u^{-1} P u P\right)+\left((P u(1-P))^{*}(P u(1-P))\right)\right) \\
& =-\operatorname{Tr}\left(-P+P u^{-1} P u P+(1-P) u^{-1} P^{2} u(1-P)\right) \\
& =-\operatorname{Tr}\left(-P+P u^{-1} P u P+(1-P) u^{-1} P u(1-P)\right) \\
& =-\operatorname{Tr}\left(P u^{-1} P u-P-(2 P-I) u^{-1} P u+(2 P-I) u^{-1} P u P\right) \\
& =-\frac{1}{4} \operatorname{Tr}\left(F\left[F, u^{-1}\right][F, u]\right) \\
& =\left\langle\operatorname{Ch} \text { odd }\left(L^{2}(M, E), \rho_{\text {std }}, 2 P-1\right),[u]\right\rangle \\
& =\left\langle\left(L^{2}(M, E), \rho_{\text {std }}, 2 P-1\right),[u]\right\rangle \\
& =\operatorname{ind}(P u P-(1-P)) \\
& =\operatorname{ind}(P u P) .
\end{aligned}
$$

The previous calculation contains a third version of the partitioned manifold theorem, which is considered in [38]. It reads as follows.

1.5.13 Theorem (Partitioned manifold index theorem). Let $(M, g)$ be a complete Riemannian spin manifold of odd dimension. Assume that $M$ is partitioned by a compact hypersurface $N \subset M$. Let

$$
\varphi_{N}: \mathrm{K}_{1}(M) \rightarrow \mathbb{Z}
$$

be the map constructed in the following way: let $A \subset \mathscr{L}_{\mathbb{C}}^{*}\left(L^{2}(M, \Sigma M)\right)$ be the $\mathrm{C}^{*}$-algebra obtained by adjoining a unit to $\mathrm{C}^{*}(M)$ and set $H_{+}:=$ $P\left(L^{2}(M, \Sigma M)\right)$. Map the class $[u] \in \mathrm{K}_{1}(M)$ represented by $u \in \mathrm{GL}_{\infty}(A)$ to the Fredholm index of Toeplitz-type operator

$$
T_{u}:\left(H_{+}\right)^{n} \rightarrow\left(H_{+}\right)^{n}
$$

obtained by first multiplying by $u$ and then compressing to $\left(H_{+}\right)^{n}$ by the orthogonal projection $P \oplus \cdots \oplus P$. Then the equality

$$
\varphi_{N}\left(\operatorname{ind}_{1}\left(D_{M}\right)\right)=\operatorname{ind}\left(D_{N}\right)
$$

holds. 


\section{Coarse index theory and positive scalar curvature}

Finally, we remark that another proof of the partitioned manifold index theorem is given in [37].

The general version of the partitioned manifold index theorem now reads as follows, see [51].

1.5.14 Theorem (Coefficient version of Roe's partitioned index theorem). Let $M$ be an odd-dimensional manifold with $\operatorname{dim}(M) \geq 3$ and $N \subset M$ a compact partitioning hypersurface, let $S$ be a Dirac bundle of Hilbert A-modules over $M$ with associated Dirac operator $D: \Gamma(M, S) \rightarrow \Gamma(M, S)$. Let

$$
\varphi_{N}: \mathrm{K}_{1}\left(\mathrm{C}^{*}(M, A)\right) \rightarrow \mathrm{K}_{0}(A) \quad, \quad[u] \mapsto \operatorname{ind}_{A}\left(T_{u}\right)
$$

be the homomorphism defined by the partitioning hypersurface. Then one has

$$
\varphi_{N}\left(\operatorname{ind}_{1}(D)\right)=\operatorname{ind}_{A}\left(\operatorname{res}_{N}(D)\right) .
$$

Here $\operatorname{res}_{N}(D)$ is the restriction of $D$ to $N$ as in Definition 1.5.8.

1.5.15 Remark. For $A=\mathbb{C}$ this is the usual partitioned index theorem as it was given by Roe.

1.5.16 Example. If $M$ is spin and $N$ carries the induced spin structure, we can identify the restricted spinor bundle $(\Sigma M)_{\mid N}$ with the intrinsic spinor bundle $\Sigma N$ and the restricted spinorial Dirac operator $\operatorname{res}_{N}\left(D_{M}\right)$ with the intrinsic spinorial Dirac operator $D_{N}$ of $N$. This remains true if one twists: the restriction of the Dirac operator $D_{M, E}=D_{M} \otimes E$, which is obtained by twisting the spinorial Dirac operator $D_{M}$ on $M$ with a Banach bundle $(E, \nabla)$ equipped with a covariant derivative operator $\nabla$, can be identified with $D_{N} \otimes E_{\mid N}$ where $E_{\mid N}$ carries the restricted covariant derivative operator $\nabla^{E_{\mid N}}$. In order to see this, we first of all notice that by definition

$$
\operatorname{res}_{N}(\Sigma M \otimes E)=(\Sigma M \otimes E)_{\mid N}=(\Sigma M)_{\mid N} \otimes\left(E_{\mid N}\right)
$$

and secondly, that the connection on $\operatorname{res}_{N}(\Sigma M \otimes E)$ is given by (1.5.1) as

$$
\nabla_{X}^{\mathrm{res}_{N}(\Sigma M \otimes E)} s_{\mid N}=\left(\nabla_{X}^{\Sigma M \otimes E} s-\frac{1}{2} \bar{c}_{M}\left(\nabla_{X}^{M} \nu\right)\left(\bar{c}_{M}(\nu)(s)\right)\right)_{\mid N}
$$

for any section $s \in \Gamma(M, \Sigma M \otimes E)$. If $s=\psi \otimes t$ is an elementary tensor section with $\psi \in \Gamma(M, \Sigma M)$ and $t \in \Gamma(M, E)$, then formula (1.5.11) becomes

$$
\begin{aligned}
\nabla_{X}^{\mathrm{res}_{N}(\Sigma M \otimes E)} s_{\mid N} & =\left(\nabla_{X}^{\Sigma M \otimes E} s-\frac{1}{2}\left(\nabla_{X}^{M} \nu\right) \cdot \nu \cdot s\right)_{\mid N} \\
& =\left(\left(\nabla_{X}^{\Sigma M} \psi\right) \otimes t+\psi \otimes \nabla_{X}^{E} t\right)_{\mid N}- \\
& \quad\left(\left(\frac{1}{2} \bar{c}_{M}\left(\nabla_{X}^{M} \nu\right)\left(\bar{c}_{M}(\nu)(\psi)\right)\right) \otimes t\right)_{\mid N} \\
& =\left(\left(\nabla_{X}^{\operatorname{res}(\Sigma M)} \psi\right) \otimes t_{\mid N}\right)+\left(\psi \otimes\left(\nabla_{X}^{E} t\right)\right)_{\mid N} \\
& =\nabla_{X}^{\operatorname{res}_{N}(\Sigma M) \otimes E_{\mid N}}(\psi \otimes t)_{\mid N} \\
& =\nabla_{X}^{\operatorname{res}_{N}(\Sigma M) \otimes E_{\mid N}} s_{\mid N} .
\end{aligned}
$$


Here we have used that $\bar{c}_{M}: T M \rightarrow \operatorname{End}(\Sigma M \otimes E)$ acts by $\bar{c}_{M}(v)=c_{M}(v) \otimes \operatorname{id}_{E}$ for each $v \in T M$. Hence, $\operatorname{res}_{N}\left(D_{M, E}\right)=D_{N, E_{\mid N}}$ follows.

1.5.17 Remark. We briefly want to compare our definition of $\operatorname{res}_{N}\left(D_{M}\right)$ with the one Higson uses in his note [19]: let $M$ be a $(2 n+1)$-dimensional manifold and $\left(S, \nabla^{S}\right)$ a Dirac bundle on $M$. Both Higson's and our Dirac operator on $N$, act on sections of $S_{\mid N}$ equipped with the Clifford multiplication of $T N$ induced by the embedding $T N \rightarrow T M_{\mid N}$, cf. [19, p. 440]. On this bundle we consider the covariant derivative operator given by Equation (1.5.1) of Definition 1.5.8. Higson chooses an arbitrary compatible connection on $S_{\mid N}$ instead, again cf. [19, p. 440]. Finally, let us compare the grading on $S_{\mid N}$ that Higson uses with the one we have chosen in Definition 1.5.8. For this we need to explain that Higson $\left[19\right.$, p. 439] makes the following additional assumption on $\left(S, \nabla^{S}\right)$ : let $\left(E_{0}, E_{1}, \ldots, E_{2 n}\right)$ be a local orthonormal frame for $M$ and set

$$
\eta:=(\sqrt{-1})^{1+n} E_{0} E_{1} \cdots E_{2 n},
$$

then it is assumed that the Dirac bundle $\left(S, \nabla^{S}\right)$ is such that

$$
\eta s=s
$$

holds for each section $s \in \Gamma(M, S)$. If this additional assumption is not already true for $\left(S, \nabla^{S}\right)$ it can be enforced following [19] as follows: one considers the self-adjoint endomorphism $\sigma \in \operatorname{End}(S)$ given by left multiplication with $\eta$ on the fibres. We can then consider the eigenbundles $S_{ \pm}=\operatorname{Eig}(\sigma ; \pm 1)$. Then $S=$ $S_{+} \oplus S_{-}$and $\nabla^{S}$ as well as $\pm D$ maps $S_{ \pm}$to $S_{ \pm}$(this follows from [31, (5.4) on p. 114 as well as (5.25) on p. 128]) and we obtain a decomposition $D=D_{+} \oplus D_{-}$. The sections of the Dirac bundle $\left(S_{+}, \nabla\right)$ satisfy the desired relation, so that we can replace (if necessary) $(S, \nabla)$ by $\left(S_{+}, \nabla\right)$. With this out of the way, Higson finally orients $N$ as $\partial M_{-}$and chooses the self-adjoint endomorphism $\varepsilon: S_{\mid N} \rightarrow S_{\mid N}$ with $\varepsilon(s)=(\sqrt{-1})^{n} E_{1} \cdots E_{2 n} s$ as grading operator for $S_{\mid N}$. If $s \in \operatorname{Eig}(\varepsilon ; \pm 1)$, then by $(*)$ one has

$$
\begin{aligned}
\pm(\sqrt{-1}) \nu s & =(\sqrt{-1}) \nu \varepsilon(s) \\
& =(\sqrt{-1})^{1+n} \nu E_{1} \cdots E_{2 n} s \\
& =\eta s \\
& =s
\end{aligned}
$$

Thus $\operatorname{Eig}(\varepsilon ; \pm 1)=\operatorname{Eig}(\nu ; \mp i)$. This explains the connection between the grading on $S_{\mid N}$ used in [19] and the grading we have choosen in Definition 1.5.8.

\subsection{A geometric application}

In this section we consider a codimension two-obstruction theorem as an example of an application of the (general version of) the vanishing theorem and the partitioned manifold index theorem. This is joint work with Hanke and Schick. This results goes back to a similar result by Gromov and Lawson [16, 


\section{Coarse index theory and positive scalar curvature}

Thm. 7.47] and describes a situation in which the non-existence of a positive scalar curvature metric on a submanifold of codimension two obstructs the existence of such a metric on the ambient manifold.

A basic problem in the application of the partitioned manifold index theorem to a geometrical situation is to extend the various structures given on the partitioning submanifold to the ambient manifold. The aforementioned application identifies a geometrical setting in which such an extension is actually possible and is described in Theorem 1.6.9.

We begin by reviewing the product formula for the Rosenberg index. This will play an important role in the proof of Theorem 1.6.9.

6.1 Product formula for the Rosenberg index. In this section we extend the definition of the Rosenberg $\alpha$-index to odd-dimensional manifolds as in [17, Sec. 3, p. 14 and Sec. 4, p. 20], using a Kasparov-type product

$$
\hat{\otimes}: \mathrm{K}_{p}\left(A_{1}\right) \times \mathrm{K}_{q}\left(A_{2}\right) \rightarrow \mathrm{K}_{p+q}\left(A_{1} \hat{\otimes} A_{2}\right) \quad, \quad p, q \in \mathbb{Z} / 2
$$

whose definition we recall next. Here and in the following $\hat{\otimes}$ denotes the (minimal) graded tensor product for $\mathrm{C}^{*}$-algebras, see [3, p. 116].

We assume the reader is familiar with the Kasparov product

$$
\hat{\otimes}_{D}: \mathrm{KK}(A, D) \times \mathrm{KK}(D, B) \rightarrow \mathrm{KK}(A, B) .
$$

and

$$
\hat{\otimes}_{D}: \operatorname{KK}\left(A_{1}, B_{1} \hat{\otimes} D\right) \times \mathrm{KK}\left(D \hat{\otimes} A_{2}, B_{2}\right) \rightarrow \mathrm{KK}\left(A_{1} \hat{\otimes} A_{2}, B_{1} \hat{\otimes} B_{2}\right),
$$

respectively. This product can be extended to a product

$$
\hat{\otimes}_{D}: \operatorname{KK}_{p}(A, D) \times \operatorname{KK}_{q}(D, B) \rightarrow \mathrm{KK}_{p+q}(A, B) \quad, \quad p, q \in \mathbb{Z} / 2
$$

between the higher KK-groups $\operatorname{KK}_{p}(A, B)$. These are defined for $p \in \mathbb{N}_{\geq 1}$ by means of the complex Clifford algebras $\mathrm{Cl}_{p}^{c}$ as

$$
\mathrm{KK}_{p}(A, B):=\operatorname{KK}\left(A, B \otimes \mathrm{Cl}_{p}^{c}\right)
$$

and one sets $\operatorname{KK}_{0}(A, B):=\mathrm{KK}(A, B)$ in addition to this. Likewise, $\mathrm{K}_{p}(A)=$ $\mathrm{KK}_{p}\left(\mathbb{C}, A \otimes \mathrm{Cl}_{p}^{c}\right)$ for $p \in \mathbb{N}_{\geq 1}$. Since we only need $\mathrm{KK}_{p}(A, B)$ for $p=0$ and $p=1$ here, we give no further details and only recall that $\mathrm{Cl}_{1}^{c}=\mathbb{C} \cdot 1 \oplus \mathbb{C} \cdot i$. Next, we explain the definition of the 'mixed Kasparov product'

$$
\hat{\otimes}_{D}^{p, q}: \mathrm{KK}_{p}(A, D) \times \mathrm{KK}_{q}(D, B) \rightarrow \mathrm{KK}_{p+q}(A, B) \quad, \quad p, q \in \mathbb{Z} / 2 .
$$

Of course, for $p, q=0$ the 'mixed product' $\hat{\otimes}_{D}^{0,0}$ is simply the usual product $\hat{\otimes}_{D}$. To define $\otimes_{D}^{p, q}$ for other values of $p$ and $q$ use $[3,18.10$, p. 181] and the exterior tensor product.

Finally, we obtain the 'K-theory version'

$$
\hat{\otimes}: \mathrm{K}_{p}\left(A_{1}\right) \times \mathrm{K}_{q}\left(A_{2}\right) \rightarrow \mathrm{K}_{p+q}\left(A_{1} \hat{\otimes} A_{2}\right) \quad, \quad p, q \in \mathbb{Z} / 2
$$


of the mixed Kasparaov product announced in (5.2) by writing $\mathbb{C}=\mathbb{C} \otimes \mathbb{C}$ and $A=A \otimes \mathbb{C}$ and the following diagram

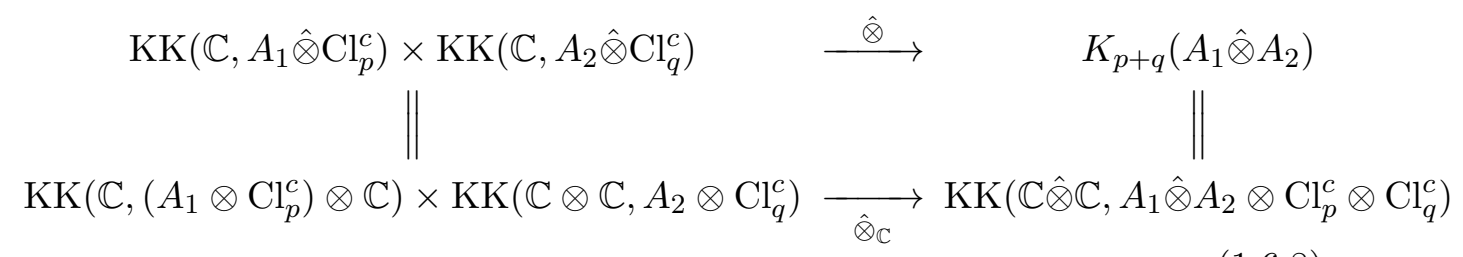

Here the lower horizontal arrow is given by (1.6.3).

Let us briefly review Schochet's Künneth formula: for a $\mathrm{C}^{*}$-algebra $A$ introduce the graded group $\mathrm{K}_{*}(A):=\mathrm{K}_{0}(A) \oplus \mathrm{K}_{1}(A)$. If $A$ and $B$ are $\mathrm{C}^{*}$-algebras we can consider the graded tensor product of $\mathrm{K}_{*}(A)$ and $\mathrm{K}_{*}(B)$. It is given by

$$
\mathrm{K}_{*}(A) \hat{\otimes} \mathrm{K}_{*}(B)=\left(\mathrm{K}_{*}(A) \otimes \mathrm{K}_{*}(B)\right)_{0} \oplus\left(\mathrm{K}_{*}(A) \otimes \mathrm{K}_{*}(B)\right)_{1}
$$

with even part

$$
\left(\mathrm{K}_{*}(A) \hat{\otimes} \mathrm{K}_{*}(B)\right)_{0}=\left(\mathrm{K}_{0}(A) \otimes \mathrm{K}_{0}(B)\right) \oplus\left(\mathrm{K}_{1}(A) \otimes \mathrm{K}_{1}(B)\right)
$$

and odd part

$$
\left(\mathrm{K}_{*}(A) \hat{\otimes} \mathrm{K}_{*}(B)\right)_{1}=\left(\mathrm{K}_{1}(A) \otimes \mathrm{K}_{0}(B)\right) \oplus\left(\mathrm{K}_{0}(A) \otimes \mathrm{K}_{1}(B)\right) .
$$

According to [42], if $A$ and $B$ are separable and $A$ is also nuclear, there exists a short exact sequence

$$
0 \rightarrow \mathrm{K}_{*}(A) \hat{\otimes} \mathrm{K}_{*}(B) \stackrel{\rho}{\rightarrow} \mathrm{K}_{*}(A \hat{\otimes} B) \stackrel{\sigma}{\rightarrow} \operatorname{Tor}\left(\mathrm{K}_{*}(A), \mathrm{K}_{*}(B)\right) \rightarrow 0 .
$$

We will not need the definition of $\rho$ and $\sigma$ here, but see [42]. (The map $\rho$ is denoted $\alpha$ in [42]. But since we are using this letter to denote the Rosenberg index we use different notation here.)

1.6.2 Theorem (Product formula). For $i=1,2$ let $M_{i}$ be a compact spin manifold and let $A_{i}$ be $\mathbb{Z} / 2$-graded $\mathrm{C}^{*}$-algebras, and let $S_{i} \rightarrow M_{i}$ be $A_{i}$-Dirac bundle with associated Dirac operator $D_{i}$. Then the exterior product $S_{1} \otimes S_{2}$ is $a\left(A_{1} \otimes A_{2}\right)$-Dirac bundle and the index ind $(D)$ of the associated Dirac operator $D_{1} \otimes D_{2}$ can be computed using the Kasparov product

$$
\hat{\otimes}: \mathrm{K}_{0}\left(A_{1}\right) \hat{\otimes} \mathrm{K}_{0}\left(A_{2}\right) \rightarrow \mathrm{K}_{0}\left(A_{1} \hat{\otimes} A_{2}\right)
$$

as

$$
\operatorname{ind}\left(D_{1} \otimes D_{2}\right)=\operatorname{ind}\left(D_{1}\right) \hat{\otimes} \operatorname{ind}\left(D_{2}\right)
$$

Proof. See [46].

1.6.3 Definition (Even and odd Rosenberg index). If $M$ is even-dimensional we set $\alpha_{0}(M):=\alpha(M)$. If $M$ is odd-dimensional, $M \times S^{1}$ is even-dimensional and we can consider $\alpha\left(M \times S^{1}\right)$. By the product formula of Theorem 1.6.2 $\alpha\left(M \times S^{1}\right)=\alpha(M) \hat{\otimes} \alpha\left(S^{1}\right)$ in $\mathrm{K}_{0}\left(\mathrm{C}^{*} \pi_{1}(M) \hat{\otimes} \mathrm{C}^{*} \pi_{1}\left(S^{1}\right)\right)$. On the other hand

$$
\left[\mathrm{K}_{0}\left(\mathrm{C}^{*} \pi_{1}(M)\right) \otimes \mathbb{Z} \cdot g_{0}\right] \oplus\left[\mathrm{K}_{1}\left(\mathrm{C}^{*} \pi_{1}(M)\right) \otimes \mathbb{Z} \cdot g_{1}\right]
$$


is isomorphic to $\mathrm{K}_{0}\left(\mathrm{C}^{*} \pi_{1}(M) \hat{\otimes} \mathrm{C}^{*} \pi_{1}\left(S^{1}\right)\right)$ via $\rho$ by Schochet's Künneth formula. Here we have written $\mathrm{K}_{0}\left(\mathrm{C}^{*} \pi_{1}\left(S^{1}\right)\right)=\mathbb{Z} \cdot g_{0}$ and $\mathrm{K}_{1}\left(\mathrm{C}^{*} \pi_{1}\left(S^{1}\right)\right)=\mathbb{Z} \cdot g_{1}$. Finally, define $\alpha_{1}(M) \in \mathrm{K}_{1}\left(\mathrm{C}^{*} \pi_{1}(M)\right)$ as the element such that $\alpha_{1}(M) \hat{\otimes} g_{1}$ is mapped onto $\alpha\left(M \times S^{1}\right)$ under the isomorphism $\rho$.

1.6.4 Lemma. Let $\mathscr{H}$ be a Hilbert A-module, which is isomorphic to the standard module $\ell^{2}(A)$. Denote by $\mathscr{Q}(\mathscr{H})=\mathscr{L}_{A}^{*}(A) / \mathscr{K}_{A}(\mathscr{H})$ the associated Calkin algebra and by $\pi: \mathscr{L}_{A}^{*}(A) \rightarrow \mathscr{Q}(\mathscr{H})$ the quotient map. If $T \in \operatorname{Fred}_{A}(\mathscr{H})$ is an A-linear Fredholm operator, i.e. the image $\pi(T) \in \mathrm{GL}\left(\mathscr{Q}_{A}(\mathscr{H})\right)$ of $T$ in the Calkin algebra is invertible, then

$$
\operatorname{ind}_{A}(T)=\partial_{1}[\pi(T)] .
$$

Here $[\pi(T)] \in \mathrm{K}_{1}(A)$ the class of $\pi(T) \in \mathrm{GL}\left(\mathscr{Q}_{A}(\mathscr{H})\right)$ and $\partial_{1}: \mathrm{K}_{1}(A) \rightarrow \mathrm{K}_{0}(A)$ is the index map.

Proof. By [48, Lemma 17.1.4, p. 270] there exists $K \in \mathscr{K}_{A}(\mathscr{H})$ such that $T+K$ admits a polar decomposition $T+K=S R$ with $S$ a partial isometry and $R$ positive and invertible such that $I-S^{*} S, I-S S^{*} \in \mathscr{K}_{A}(\mathscr{H})$ are compact projections. In particular, it follows that

$$
\pi(T)=\pi(T+K)=\pi(S) \pi(R)
$$

is invertible and since $R$ is invertible, $\pi(S)$ is invertible. For $0 \leq t \leq 1$ set

$$
R_{t}:=S(t R+(1-t) I) .
$$

Then $R_{0}=S$ and $R_{1}=S R=T+K$. For any $0 \leq t \leq 1$, the operator

$$
\pi\left(R_{t}\right)=\pi(S) \pi(t R+(1-t) I)
$$

is invertible. Hence $\pi(S) \sim_{h} \pi(T+K)=\pi(T)$ are homotopic in $\operatorname{GL}\left(\mathscr{Q}_{A}(\mathscr{H})\right)$. From this we finally obtain

$$
\begin{aligned}
\partial_{1}[\pi(T)] & =\partial_{1}[\pi(T+K)] \\
& =\partial_{1}[\pi(S)] \\
& =\left[I-S^{*} S\right]-\left[I-S S^{*}\right] \\
& =\operatorname{ind}_{A}(T) .
\end{aligned}
$$

This completes the proof.

1.6.5 Lemma. Let $M$ be a compact Riemannian spin manifold with fundamental group $\pi$. Then the Roe $\mathrm{C}^{*}$-algebra $\mathrm{C}^{*}\left(M ; \mathrm{C}^{*} \pi\right)$ coincides with the $\mathrm{C}^{*}$ algebra $\mathscr{K}_{\mathrm{C}^{*} \pi}\left(L^{2}(M, S)\right)$ of all $\mathrm{C}^{*} \pi$-compact operators. Furthermore, the even Roe indices (if $M$ is even dimensional)

$$
\operatorname{ind}_{0}\left(D_{M}\right) \in \mathrm{K}_{0}\left(\mathscr{K}_{\mathrm{C}^{*} \pi}\left(L^{2}(M, S)\right)\right)
$$

coincides with the Rosenberg $\alpha$-indices

$$
\alpha_{0}(M)=\operatorname{ind}_{\mathrm{C}^{*} \pi}\left(D_{M}\right) \in \mathrm{K}_{p}\left(\mathscr{K}_{\mathrm{C}^{*} \pi}\left(L^{2}(M, S)\right)\right) .
$$


Proof. The invariance of the generalized Fredholm index with respect to the application of normalizing functions as well as unitary invariance of the generalized Fredholm index, and Lemma 1.6.4 allows us to make the following computation

$$
\begin{aligned}
\alpha_{0}(M) & =\operatorname{ind}_{\mathrm{C}^{*} \pi}\left(D_{M}^{+}\right) \\
& =\operatorname{ind}_{\mathrm{C}^{*} \pi}\left(\chi\left(D_{M}^{+}\right)\right) \\
& =\operatorname{ind}_{\mathrm{C}^{*} \pi}\left(U^{*} \chi\left(D_{M}^{+}\right)\right) \\
& =\partial_{1}\left[\pi\left(U^{*} \chi\left(D_{M}^{+}\right)\right)\right] \\
& =\operatorname{ind}_{0}\left(D_{M}\right)
\end{aligned}
$$

This completes the proof.

1.6.6 Remark. A statement analogous to Lemma 1.6.5 should be true for the odd Roe index. A similar result can be found in [39]. We defer to give a proof here.

6.7 Extending the Mishchenko bundle. Let $M$ be a spin manifold with spinor bundle $\Sigma M, A=\mathrm{C}^{*}\left(\pi_{1}(M)\right)$ the group $C^{*}$-algebra of the fundamental group $\pi_{1}(M)$ of $M$, and $S$ the Dirac bundle given by $\Sigma M \otimes \mathscr{V}_{M}$ with $\mathscr{V}_{M}:=$ $\widetilde{M} \times{ }^{\pi_{1}(M)} A$. In Proposition 1.5.9 we saw that the restrition of the Dirac operator on $M$ to $N$ can be identified with the intrinsic Dirac operator of $N$ (with the induced spin structure). Now as we take the fundamental group into account we can no longer expect that the Dirac operator twisted by the Mishchenko line bundle on $M$ will restrict to the corresponding operator on $N$ simply because it may happen that the fundamental group $\pi_{1}(N)$ of $N$ is trivial although $\pi_{1}(M)$ might not be trivial. In order to apply Roe's index theorem for partitioned manifolds one needs an extension of the Mishchenko line bundle on the partitioning manifold to the partitioned ambient manifold. This is a non-trivial matter since such an extensions does not always exist. The following theorem due to Hanke and Schick describes a situation where such an extension is possible.

1.6.8 Definition. The double of a manifold $M$ with boundary $N=\partial M$ is $\mathbf{D}(M)=M \cup_{N} M$. If $M$ is a manifold without boundary and $W \subset M$ is a 0codimensional submanifold with boundary, we set $\mathbf{D}(M, W):=\mathbf{D}(M \backslash \operatorname{int}(W))$.

The reader who is interested in the detailed construction of the double of a manifold, can consult [27, Chapter VI, Section 5].

1.6.9 Theorem (Hanke, Schick). Let $M$ be a connected closed manifold with $\operatorname{dim}(M) \geq 3$ and $W \subset M$ a connected submanifold of codimension 0 with boundary $\partial W$. Additionaly, assume that the following holds:

(1) The boundary $\partial W$ is connected.

(2) The second homotopy group of $M$ vanishes: $\pi_{2}(M)=0$.

(3) The Hurewicz map hur: $\pi_{1}(\partial W) \rightarrow H_{1}(\partial W)$ becomes injective when restricted to the kernel $\operatorname{ker}\left(i_{*}\right) \subset \pi_{1}(\partial W)$ of the map induced by inclusion map $i: \partial W \rightarrow W$. 
1. Coarse index theory and positive scalar curvature

(4) The inclusion map $j: W \rightarrow M$ induces a monomorphism $\pi_{1}(j): \pi_{1}(W) \rightarrow$ $\pi_{1}(M)$ whose image has infinite index.

Then the following holds:

(a) Let $p: \bar{M} \rightarrow M$ be the covering corresponding to the subgroup $j_{*}\left(\pi_{1}(W)\right)$ of $\pi_{1}(M)$, and $\bar{W} \subset \bar{M}$ be a lift of $W$ to $\bar{M}$. If we consider the manifold $\mathbf{D}(\bar{M}, \bar{W})$ which is partitioned by $\partial \bar{W}$ of $\bar{W}$, then there exists an extension of the Mishchenko line bundle $\mathscr{V}_{\partial \bar{W}}$ over $\partial \bar{W}$ to a flat bundle $\mathscr{E}$ over $\mathbf{D}(\bar{M}, \bar{W})$

(b) In the case where $W=N \times \mathbf{D}^{2}$ is a trivial tubular neighbourhood of a connected ${ }^{13}$ submanifold $N \subset M$ with $\operatorname{codim}(N)=2$, the manifold $M$ admits no metric of positive scalar curvature if either (i) $M$ is odd-dimensional and $\alpha_{1}(N) \neq 0$, or if (ii) $M$ is even-dimensional and $\alpha_{0}(N) \neq 0$. Here $\alpha_{0}(N)$ and $\alpha_{1}(N)$ is the even and odd Rosenberg index of $N$, respectively, as it is defined in Definition 1.6.3.

Proof. Let $p: \bar{M} \rightarrow M$ be the covering corresponding to the subgroup $j_{*}\left(\pi_{1}(W)\right)$ of $\pi_{1}(M)$. With this choice we can, by standard covering theory, lift the inclusion map $j: W \rightarrow M$ to an injection $\bar{j}: W \rightarrow \bar{M}$, and $\bar{j}_{*}: \pi_{1}(W) \rightarrow \pi_{1}(\bar{M})$ is bijective. Set $\bar{W}:=\bar{j}(W)$.

(a) Let $k: \partial \bar{W} \rightarrow \bar{M} \backslash \bar{W}$ be the inclusion map. Consider

$$
k_{*}: \pi_{1}(\partial \bar{W}) \rightarrow \pi_{1}(\bar{M} \backslash \bar{W})
$$

Subclaim: The following hold:

(i) $k_{*}$ is injective.

(ii) There exists $r: \pi_{1}(\bar{M} \backslash \bar{W}) \rightarrow \pi_{1}(\partial \bar{W})$ with $r \circ k_{*}=\operatorname{id}_{\pi_{1}(\partial \bar{W})}$.

With other words, $k_{*}$ is a split injection.

Ad (i): Applying the functor $\pi_{1}$ to the commutative diagram

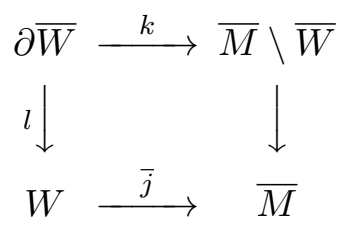

in which the vertical maps are given by inclusions and the covering projection $p$ (thus $l=i \circ p_{\mid \partial \bar{W}}^{\mid W}$ ), we obtain the commutative diagram

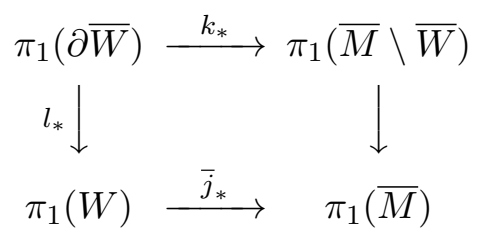

\footnotetext{
${ }^{13}$ Such that $\partial W=N \times S^{1}$ satisfies assumption (1)
} 


\section{Coarse index theory and positive scalar curvature}

The map $\bar{j}_{*}$ is injective. Indeed, $j_{*}=p_{*} \circ \bar{j}_{*}$ and $j_{*}$ is injective by assumption (4). Thus $\operatorname{ker}\left(k_{*}\right) \subset \operatorname{ker}\left(l_{*}\right)$. Therefore, if $[\alpha] \in \operatorname{ker}\left(k_{*}\right)$, then the path $\alpha$ is both nullhomotopic as a map to $\bar{W}$ and as a map to $\bar{M} \backslash \bar{W}$. Consequently, there exists a singular sphere $\sigma: S^{2} \rightarrow \bar{M}$ which maps the lower and upper semisphere $S_{-}^{2}$ and $S_{+}^{2}$, into $\bar{W}$ and $\bar{M} \backslash \bar{W}$, respectively, and the equator $S^{1} \subset S^{2}$ onto the curve $\operatorname{im}(\alpha)$. We have $\sigma_{*}\left[S^{2}\right]=0$ because of $\pi_{2}(\bar{M})=\pi_{2}(M)=0$ by assumption (2). But $\partial^{\operatorname{MVS}}\left(\sigma_{*}\left[S^{2}\right]\right)=\alpha_{*}\left[S^{1}\right]=\operatorname{hur}_{1}[\alpha]$ by construction of the boundary operator $\partial^{\mathrm{MVS}}$ of the Mayer-Vietoris sequence of the triad $(\bar{M}, \bar{M} \backslash \bar{W}, \bar{W})$. The diagram

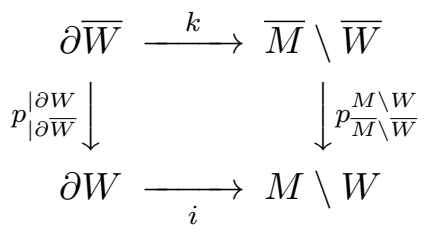

commutes. Because of this $\left(p^{\mid \partial W}\right)_{*}$ maps $\operatorname{ker}\left(k_{*}\right)$ into $\operatorname{ker}\left(i_{*}\right)$ and the diagram

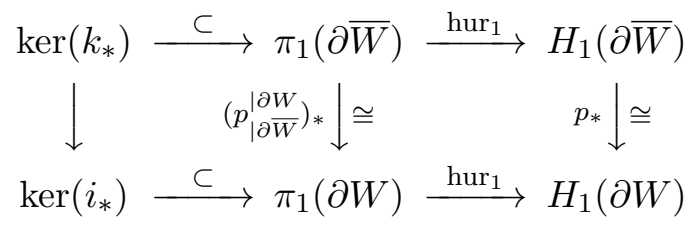

commutes because the Hurewicz morphism is natural. By assumption (3) the lower horizontal composition in this diagram is injective, and therefore the same is also true for the upper horizontal one. Thus $[\alpha]=0$, proving that $k_{*}$ is injective.

Ad (ii): By considering the long exact sequence of the pair $(\bar{M}, \bar{W})$ one finds $H_{1}(\bar{M}, \bar{W})=0$ because the inclusion $l$ is an isomorphism on zeroth homology as both $\bar{M}$ and $\bar{W}$ are path connected.

The pair $(\bar{M}, \bar{W})$ is 1-connected and the relative Hurewicz theorem as well as assumption (2) imply $H_{2}(\bar{M}, \bar{W})=0$.

Thus the groups $H_{1}(\bar{M} \backslash \bar{W}, \partial \bar{W})$ and $H_{2}(\bar{M} \backslash \bar{W}, \partial \bar{W})$ vanish (use excision and homotopy invariance). Therefore $H_{1}(k): H_{1}(\partial \bar{W}) \rightarrow H_{1}(\bar{M} \backslash \bar{W})$ is an isomorphism. The diagram

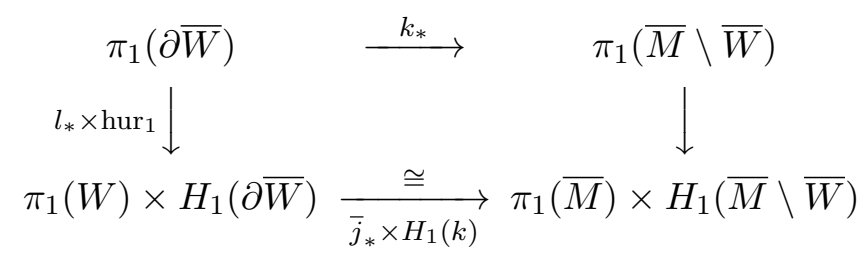

obtained from Diagram 1.6.9 by taking the products with the Hurewicz morphism commutes, and the lower horizontal arrow is an isomorphism since both $\bar{j}_{*}$ and $H_{1}(k)$ are isomorphism.

The map $l_{*} \times$ hur $_{1}$ is injective. Indeed, if $[\alpha] \in \pi_{1}(\partial \bar{W})$ lies in the kernel of this map, then $[\alpha]$ lies in both the kernel of $l_{*}$ and the kernel of hur ${ }_{1}$. But 
$l=i \circ p_{\mid \partial \bar{W}}^{\mid \partial W}$ and thus $[\alpha]$ must lie in $\left(p_{\mid \partial \bar{W}}^{\mid \partial W}\right)^{-1}\left(\operatorname{ker}\left(i_{*}\right)\right)=\operatorname{ker}\left(k_{*}\right)$. But by assumption (3) hur ${ }_{1}$ is injective on $\operatorname{ker}\left(k_{*}\right)$ as we noticed before. Hence $[\alpha]=1$, proving that $l_{*} \times$ hur $_{1}$ is injective. Thus we can regard $\pi_{1}(\partial \bar{W})$ as subgroup of $\pi_{1}(\bar{M}) \times H_{1}(\bar{M} \backslash \bar{W})$ via the injection given by the composition of this injection with the lower horizontal arrow. The right vertical arrow then surjects onto this subgroup of $\pi_{1}(\bar{M}) \times H_{1}(\bar{M} \backslash \bar{W})$ and gives a retraction $r$ of $k_{*}$.

Finally, we remark that the diagram

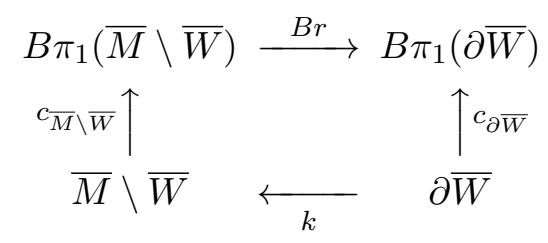

in which the vertical maps are given by the classifying maps of the corresponding universal coverings, commutes up to homotopy exactly if the diagram

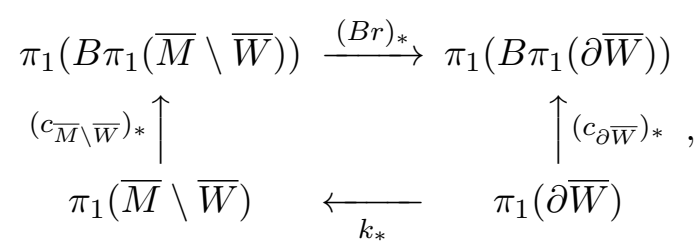

applying the functor $\pi_{1}$, commutes, see [47, Section 8.7 and 8.8]. But this follows directly from $r \circ k_{*}=\mathrm{id}_{B \pi_{1}(\partial \bar{W})}$. Indeed, if $X$ is any space, then under the identification of $\pi_{1}\left(B \pi_{1}(X)\right)$ with $\pi_{1}(X)$ via the boundary operator in the long exact sequence of the fibration $E \pi_{1}(X) \rightarrow B \pi_{1}(X)$, the map induced on $\pi_{1}$-level by $c_{X}: X \rightarrow B \pi_{1}(X)$ is identified with $\operatorname{id}_{B \pi_{1}(X)}$. Therefore, if we set $\mathscr{E}:=\left(B r \circ c_{\bar{M} \backslash \bar{W}}\right)^{*}\left(E \pi_{1}(\partial \bar{W})\right)$, then $k^{*} \mathscr{E} \cong \mathscr{V} \partial \bar{W}$ by the Homotopy Theorem, see [47, Thm. 14.3.3]. This completes the proof of assertion (a).

(b) We consider the cases where $M$ is odd- and even-dimensional, respectively, seperately, beginning with the odd-dimensional case.

(i) Assume $M$ is odd dimensional. Denote by $W$ a trivial normal neighbourhood of $N$. Then $W$ is a zero-codimensional submanifold of $M$. The image $\bar{W}$ of $W$ under $\bar{j}$ is a zero-codimensional submanifold of $\bar{M}$. The manifold $\mathbf{D}(\bar{M}, \bar{W})$ admits a spin structure and is partitioned by the boundary $\partial \bar{W} \cong N \times S^{1}$ of $\bar{W}$. By part (a) there is a flat bundle $\mathscr{E}$ over $\mathbf{D}(\bar{M}, \bar{W})$ which extends the Mishchenko line bundle $\mathscr{V}$ over $\partial \bar{W}$. By Theorem 1.5.14 and Example 1.5.16 we have:

$$
\zeta_{\partial \bar{W}}\left(\operatorname{ind}_{1}\left(D_{\mathbf{D}(\bar{M}, \bar{W}), \mathscr{E}}\right)\right)=\operatorname{ind}_{0}\left(D_{\partial \bar{W}, \mathscr{V}}\right) \in \mathrm{K}_{0}\left(\mathrm{C}^{*}\left(\pi_{1}(\partial \bar{W})\right)\right) .
$$

Furthermore, we have by Lemma 1.6.5

$$
\begin{aligned}
\operatorname{ind}_{0}\left(D_{\partial \bar{W}, \mathscr{V}}\right) & =\alpha_{0}(\partial \bar{W}) \\
& =\alpha_{0}(\partial W) \\
& =\alpha_{0}\left(N \times S^{1}\right) \\
& =\rho^{-1}\left(\alpha_{1}(N) \hat{\otimes} g_{1}\right)
\end{aligned}
$$


since $W \cong \bar{W}$ and because of the product formula for the Rosenberg $\alpha$-index. (Here $g_{1}$ is such that $\mathrm{K}_{1}\left(\mathrm{C}_{\max }^{*}(\mathbb{Z})\right)=\mathbb{Z} \cdot g_{1}$. See Definition 1.6.3.) In conclusion,

$$
\rho\left(\zeta_{\partial \bar{W}}\left(\operatorname{ind}_{1}\left(D_{\mathbf{D}(\bar{M}, \bar{W}), \mathscr{E}}\right)\right)\right)=\alpha_{1}(N) \hat{\otimes} g_{1}
$$

and hence $\operatorname{ind}_{1}\left(D_{\mathbf{D}(\bar{M}, \bar{W}), \mathscr{E}}\right) \neq 0$ as we assume $\alpha_{1}(N) \neq 0$. Since the bundle $\mathscr{E}$ is flat this and Lemma 1.3.9 imply that $\mathbf{D}(\bar{M}, \bar{W})$ has no PSC-metric outside of a compact subset. Now, if $M$ would admit a metric of positive scalar curvature then $\bar{M}$ would admit a metric of uniformly positive scalar curvature which in turn would imply that $\mathbf{D}(\bar{M}, \bar{W})$ admits a metric with uniformly positive scalar curvature outside of a compact neighbourhood of $\partial W$ which contradicts $\operatorname{ind}_{1}\left(D_{\mathbf{D}(\bar{M}, \bar{W}), \mathscr{E}}\right) \neq 0$.

(ii) Assume now that $M$ is even-dimensional. In this case we replace the pair $(M, N)$ by $\left(M \times S^{1}, N \times S^{1}\right)$. Since $N$ has trivial normal bundle in $M$ the normal bundle of $N \times S^{1}$ in $M \times S^{1}$ is trivial. Also the fundamental group of the submanifold still injects into the fundamental group of the ambient manifold. Since

$$
\begin{aligned}
\alpha_{1}\left(N \times S^{1}\right) \neq 0 & \Longleftrightarrow \alpha_{1}(N) \hat{\otimes} g_{1} \neq 0 \\
& \Longleftrightarrow \rho^{-1}\left(\alpha_{1}(N) \hat{\otimes} g_{1}\right)=\alpha(N) \hat{\otimes} \alpha\left(S^{1}\right) \neq 0 \\
& \Longrightarrow \alpha(N) \neq 0
\end{aligned}
$$

it follows from (i) that $M \times S^{1}$ admits no metric of postive scalar curvature. Hence $M$ has no such metric. 


\section{A counterexample to a conjecture about positive scalar curvature}

\subsection{Introduction}

The Gromov-Lawson-Rosenberg Conjecture gives a necessary and sufficient condition in terms of an index theoretic obstruction for a closed connected spin manifold (of dimension $\geq 5$ ) to admit a Riemannian metric of positive scalar curvature. An analogous conjecture in the non-spin case is not known, though a result of Gromov-Lawson-Stolz shows that each simply connected non-spin manifold admits a Riemannian metric of positive scalar curvature. In [7] Chang has proposed a conjecture which gives a necessary and sufficient condition in terms of a homological obstruction for a closed connected totally non-spin manifold (of dimension $\geq 5$ ) to admit a Riemannian metric of positive scalar curvature. Here a totally non-spin manifold is one for which neither the manifold nor its universal covering admits a spin structure. Chang attributes the conjecture to Rosenberg and Weinberger. In this chapter we show that the counterexample from [41] to the (unstable) Gromov-Lawson-Rosenberg Conjecture can be enhanced to give a counterexample to the general version of the Chang-RosenbergWeinberger Conjecture.

\subsection{Preliminary remarks}

2.1 Smooth connected sums. Let $M$ and $N$ be connected smooth manifolds of the same dimension $n$. A detailed construction of the smooth connected sum $M \# N$, including a proof that it is again a smooth manifold, can be found in [27, pp. 90-92]. It is useful to know that the fundamental group and also the homology groups of $M \# N$ can be expressed completely in terms of those of $M$ and $N$. For example one has $\pi_{1}(M \# N)=\pi_{1}(M) * \pi_{1}(N)$ for the fundamental groups if $n \geq 3$, see [27, p. 94] or [45], and similarly $H_{p}(M \# N) \cong H_{p}(M) \oplus H_{p}(N)$ for the homology groups in degree $1 \leq p \leq n-1$, see [10] or [27, p. 94]. Of course, $H_{0}(M \# N) \cong H_{n}(M \# N) \cong \mathbb{Z}$, if we assume that $M$ and $N$ are orientable. Sometimes we want to prevent a manifold from admitting a spin structure (without changing the fundamental group). This can be achieved by taking the connected sum with a suitable non-spinable manifold as the following lemma shows.

2.2.2 Lemma. Let $M_{1}$ and $M_{2}$ be closed, oriented manifolds of the same dimension $n \geq 3$. Then $M_{1} \# M_{2}$ is spin if and only if both $M_{1}$ and $M_{2}$ are spin. 
Proof. " $\Rightarrow$ ": Since $n \geq 3$ the trivial spin structure is the only spin structure on $S^{n-1}$. By removing suitable closed discs $D_{1}$ and $D_{2}$ from $M_{1}$ and $M_{2}$, respectively, we can regard $M_{1} \backslash D_{1}$ and $M_{2} \backslash D_{2}$ as open submanifolds of $M_{1} \# M_{2}$. Of course, these inherit spin structures from $M_{1} \# M_{2}$. These spin structures in turn induce a spin structures on $\partial D_{1} \subset M_{1}$ and $\partial D_{2} \subset M_{2}$, which can be extended to the whole of $M_{1}$ and $M_{2}$ trivially.

"६": See [1] or [31, p. 91, Remark 2.17].

If $M$ and $N$ are connected smooth manifold, then $X:=M \# N$, for $N$ simplyconnected has the same fundamental group as $M$ according to paragraph 2.1. Furthermore, the universal covering of $M \# N$ can be obtained from the universal covering of $M$ in the following easy manner: First, we can assume that $M \# N$ is obtained by gluing $N$ to $M$ by identifying a disc in $N$ with a disc $M$ which itself lies completely in a neighbourhood which is uniformly covered by the covering map from $\bar{M}$ onto $M$. Then $\overline{M \# N}$ is $\bar{M}$ with a copy of $N$ attached in the obvious way to every sheet over the chosen uniformly covered neighbourhood.

2.3 Spin structures and covering spaces. Let $M$ be a connected closed manifold and $p: \bar{M} \rightarrow M$ its universal covering. We can ask whether a spin structure on $M$ ascends to a spin structure on $\bar{M}$, and vice versa, whether a spin structure on $\bar{M}$ descends to one on $M$. The latter is false: If $\bar{M}$ is spin, then $M$ needs not to be spinable, not even orientable, as one can see by looking at real projective spaces. A sufficient criterion to decide whether a spin structure on $\bar{M}$ induces a spin structure on $M$ can be found, e.g., in [14, Proposition 1.4 .2 , p. 28]. On the other hand, it is true that a spin structure on $M$ always ascends to one on $\bar{M}$ (this is even true for any covering, not only the universal one). If $M$ itself admits no spin structure one can at least ask if there is one on $\bar{M}$. If this is the case, one calls $M$ almost spin, see [32, Definition 1.2, p. 104]. Thus, e.g., real projective spaces are almost spin. If not even $\bar{M}$ admits a spin structure (and hence the same must be true for $M$ ), one calls $M$ totally non-spin, see [7, p. 1621].

2.2.4 Example (Examples of totally non-spin manifolds). Any simplyconnected (and hence orientable) non-spin manifold, e.g. $\mathbb{C P}^{2 n}$ or $\mathrm{SU}(3) / \mathrm{SO}(3)$. For the latter, see $[12$, p. 50 f.]. On the other hand no manifold of dimension less or equal to 3 is totally non-spin.

\subsection{The counterexample}

In this section we give a counterexample to the following conjecture from [7].

2.3.1 Conjecture. Suppose that $M$ is a totally non-spin manifold with fundamental group $\Gamma$ and dimension $n=\operatorname{dim}(M)$ at least five. Let $f: M \rightarrow \underline{B} \Gamma$ be the composition of the classifying map $c: M \rightarrow B \Gamma$ of the universal covering of $M$, and the natural map $B \Gamma \rightarrow \underline{B} \Gamma$. Denote by $[M]$ the fundamental class of $M$ in $H_{n}(M)$. Then $M$ admits a metric of positive scalar curvature if and only if $f_{*}[M]$ vanishes in $H_{n}(\underline{B} \Gamma)$. 
Here $B \Gamma$ is the classifying space for the group $\Gamma$ and $\underline{B} \Gamma$ is the classifying space for proper actions, cf. [7, p. 1623].

Our counterexample is based on Schick's counterexample to the (unstable) Gromov-Lawson-Rosenberg conjecture, given in [41]. There a 5-dimensional closed spin-manifold $M$ with fundamental group $\Gamma=\mathbb{Z}^{4} \oplus \mathbb{Z} / 3$ is constructed, whose Rosenberg index vanishes, but which nevertheless does not admit a metric of positive scalar curvature. By taking the connected sum of this manifold $M$ with any simply-connected non-spin manifold, we obtain a totally non-spin manifold, which has the same fundamental group as $M$. Therefore $B \Gamma=B\left(\mathbb{Z}^{4}\right) \times B(\mathbb{Z} / 3)=\mathbb{T}^{4} \times B(\mathbb{Z} / 3)$ and analogously $\underline{B} \Gamma=\mathbb{T}^{4}$ by $[7,(1)$ and (4), p. 1624]. Specifically, $H_{n}(\underline{B} \Gamma)=0$ for $n \geq 5$, so that the condition on $f_{*}[X]$ from Conjecture 2.3.1 is satisfied in the case at hand. The argument in [41] relies on the following observation by Stolz, and we will also make significant use of this result.

2.3.2 Lemma. Let $X$ be a topological space. Denote for any $n \in \mathbb{N}_{\geq 2}$ by $H_{n}^{+}(X)$ the set of all homology classes $f_{*}[M] \in H_{n}(X)$ where $M$ is an n-dimensional manifold which admits a metric of positive scalar curvature, and $f: M \rightarrow X$ is a continuous map. Then for any class $u \in H^{1}(X)$ the map

$$
u \cap: H_{n}(X) \rightarrow H_{n-1}(X)
$$

maps $H_{n}^{+}(X)$ into $H_{n-1}^{+}(X)$ if $3 \leq n \leq 8$.

Proof. See [41] for $3 \leq n \leq 7$ and [24, Thm 4.4] for $n=8$.

2.3.3 Proposition. Let $M$ be the manifold constructed in [41] and $N$ a simply connected manifold of dimension 5, which admits no spin structure. Then the manifold $X:=M \# N$ has non-spin universal covering and admits no metric with positive scalar curvature.

Proof. First of all, if $X$ is constructed as above, we have already noted that it has non-spin universal covering. To obtain an explicit simply-connected nonspin 5-manifold $N$, one can start with $\mathbb{C} P^{2} \times S^{1}$, which is non-spin as $\mathbb{C} P^{2}$ is, and then do surgery on the embedded $S^{1}$ to obtain the simply-connected $N$. Because this surgery does not touch the embedded $\mathbb{C} P^{1}$ with its non-spin normal bundle, the resulting $N$ remains a non-spin manifold.

In order to see that $X$ admits no metric of positive scalar curvature, we use the same argument as in [41]. To begin with, we choose the model $B \Gamma=\mathbb{T}^{4} \times B \mathbb{Z} / 3$. Recall,

$$
H_{n}\left(\mathbb{T}^{d}\right)=\mathbb{Z}^{d(n)} \quad, \quad d(n)=\left(\begin{array}{l}
d \\
n
\end{array}\right)
$$

and

$$
H_{n}(B \mathbb{Z} / k)=H_{n}(\mathbb{Z} / k)= \begin{cases}\mathbb{Z}, & n=0 \\ \mathbb{Z} / k, & n \text { odd } \\ 0, & n \text { even }\end{cases}
$$


Together with the Künneth formula this gives

$$
H_{k}(B \Gamma)=\bigoplus_{p_{1}+\cdots+p_{5}=k} H_{p_{1}}\left(X_{1}\right) \otimes \cdots \otimes H_{p_{4}}\left(X_{4}\right) \otimes H_{p_{5}}\left(X_{5}\right) .
$$

Here we have written $\mathbb{T}^{4}=X_{1} \times \cdots \times X_{4}$ as product of four copies of $\mathbb{T}$, and $X_{5}$ for $B \mathbb{Z} / 3$.

Fix a basepoint $x=\left(x_{1}, \ldots, x_{5}\right) \in B \Gamma$ and let $p: \mathbb{T} \rightarrow B \mathbb{Z} / 3$ be a map which induces an epimorphism on $\pi_{1}$ as in [41], as well as $f_{j}: X_{j} \rightarrow B \Gamma$ the map which includes $X_{j}$ identically and basepoint-preserving. We denote by $[*] \in$ $H_{0}(B \Gamma)$ the canonical generator. Next, choose for each $1 \leq j \leq 4$ generators $g_{j} \in H_{1}\left(X_{j}\right)$ and elements $g_{j}^{*} \in H^{1}\left(X_{j}\right)$ with $\left\langle g_{j}^{*}, g_{j}\right\rangle=1$, and let $g_{5} \in H_{1}\left(X_{5}\right)$ be $p_{*}[\mathbb{T}]$ where $[\mathbb{T}]$ is the standard generator for $H_{1}(\mathbb{T})$. Introduce the elements $v_{j}:=\left(f_{j}\right)_{*}\left(g_{j}\right) \in H_{1}(B \Gamma)$ for $j=1, \ldots, 5$ as well as $a_{1}, \ldots, a_{4} \in H^{1}(B \Gamma)$ with

$$
\begin{aligned}
& a_{1}:=\left(\mathrm{pr}_{1}\right)^{*}\left(g_{1}^{*}\right) \times 1 \times 1 \times 1 \times 1, \\
& a_{2}:=1 \times\left(\mathrm{pr}_{2}\right)^{*}\left(g_{2}^{*}\right) \times 1 \times 1 \times 1, \\
& a_{3}:=1 \times 1 \times\left(\mathrm{pr}_{3}\right)^{*}\left(g_{3}^{*}\right) \times 1 \times 1, \\
& a_{4}:=1 \times 1 \times 1 \times\left(\mathrm{pr}_{4}\right)^{*}\left(g_{4}^{*}\right) \times 1
\end{aligned}
$$

Finally, set

$$
w:=v_{1} \times \cdots \times v_{4} \times v_{5} \in H_{5}(B \Gamma)
$$

and

$$
z:=[*] \times[*] \times[*] \times v_{4} \times v_{5} \in H_{2}(B \Gamma) .
$$

By the Künneth formula, $w \neq 0$ and $z \neq 0$. Furthermore,

$$
z=a_{1} \cap\left(a_{2} \cap\left(a_{3} \cap w\right)\right) \in H_{2}(B \Gamma) .
$$

For example one has

$$
\begin{aligned}
a_{3} \cap w & =\left(\left(1 \times 1 \times\left(\mathrm{pr}_{3}\right)^{*}\left(g_{3}^{*}\right)\right) \times(1 \times 1)\right) \cap\left(\left(v_{1} \times v_{2} \times v_{3}\right) \times\left(v_{4} \times v_{5}\right)\right) \\
& =\left(\left(1 \times 1 \times\left(\mathrm{pr}_{3}\right)^{*}\left(g_{3}^{*}\right)\right) \cap\left(v_{1} \times v_{2} \times v_{3}\right)\right) \times\left((1 \times 1) \cap\left(v_{4} \times v_{5}\right)\right) \\
& =\left(\left(1 \cap v_{1}\right) \times\left(1 \cap v_{2}\right) \times\left(\left(\mathrm{pr}_{3}\right)^{*}\left(g_{3}\right)^{*} \cap v_{3}\right)\right) \times\left(\left(1 \cap v_{4}\right) \times\left(1 \cap v_{5}\right)\right) \\
& =v_{1} \times v_{2} \times\left(\left(\operatorname{pr}_{3}\right)^{*}\left(g_{3}^{*}\right) \cap v_{3}\right) \times v_{4} \times v_{5} \\
& =v_{1} \times v_{2} \times[*] \times v_{4} \times v_{5},
\end{aligned}
$$

because of $\left(\mathrm{pr}_{3}\right)^{*}\left(g_{3}^{*}\right) \cap\left(i_{3}\right)_{*}\left(g_{3}\right)=\left\langle g_{3}^{*}, g_{3}\right\rangle[*]$. Let $f: \mathbb{T}^{5} \rightarrow \mathbb{T}^{4} \times B \mathbb{Z} / 3$ be given by $f=\left(f_{1} \times f_{2} \times f_{3} \times f_{4}\right) \times\left(f_{5} \circ p\right)$ and choose $\left(g_{1} \times \cdots \times g_{4}\right) \times[\mathbb{T}]=$ : $\left[\mathbb{T}^{5}\right]$ as fundamental class for $\mathbb{T}^{5}$. Then $f_{*}\left[\mathbb{T}^{5}\right]=w$. As in [41] one can construct a bordism in $\Omega_{5}^{\mathrm{spin}}(B \Gamma)$ from $f$ to a map $g: M \rightarrow B \Gamma$ which induces an isomorphism on $\pi_{1}$-level. This defines the manifold $M$. Now let $N$ be any simply-connected closed non-spin manifold of dimension 5 and set $X:=M \# N$.

Finally, assume that $X$ admits a metric of positive scalar curvature. Then consider the map $h: M \sqcup N \rightarrow B \Gamma$ on the disjoint union of $M$ and $N$, which 
equals $g$ on $M$ and sends $N$ to a point. One has $h_{*}[M \sqcup N]=g_{*}[M]=w$ and since $M \sqcup N$ is bordant to $M \# N$, it follows that $w \in H_{5}^{+}(X)$. But then it follows from $(*)$ as well as Lemma 2.3.2 that $w$ is mapped to $z$ under the following map

$$
\begin{aligned}
H_{5}^{+}(B \Gamma) & \stackrel{a_{3} \cap}{\longrightarrow} H_{4}^{+}(B \Gamma) \\
& \stackrel{a_{2} \cap}{\longrightarrow} H_{3}^{+}(B \Gamma) \\
& \stackrel{a_{1} \cap}{\longrightarrow} H_{2}^{+}(B \Gamma) .
\end{aligned}
$$

Hence $z=k_{*}\left[S^{2}\right]$ for some $k: S^{2} \rightarrow B \Gamma$ since $S^{2}$ is the only orientable surface which admits a metric of positive scalar curvature. On the other hand, $\pi_{2}(B \Gamma)=0$ so that $k$ is null homotopic. This implies $z=0$, which is a contradiction. 


\section{A. Appendix}

\subsection{Some technical lemmas}

In this section we prove a few technical results, which will be useful in the main part.

1.1.1 Lemma (Technical lemma). If one chooses for each $\varepsilon>0$ real numbers $0<R_{\varepsilon}<S_{\varepsilon}$ with $R_{\varepsilon} \rightarrow \infty$ as $\varepsilon \rightarrow 0$ as well as $S_{\varepsilon}-R_{\varepsilon}=C=$ const., then there exist functions $\Phi_{\varepsilon} \in C_{\mathrm{cpt}}^{\infty}(\mathbb{R})$ satisfying the following:

(i) $0 \leq \Phi_{\varepsilon} \leq 1$.

(ii) $\Phi_{\varepsilon}$ vanishes outside of $\left[-S_{\varepsilon}, S_{\varepsilon}\right]$ and is constantly equal to 1 on $\left[-R_{\varepsilon}, R_{\varepsilon}\right]$.

(iii) There exists a uniform bound for the first and second derivative of $\Phi_{\varepsilon}$, i.e. $\left\|\Phi_{\varepsilon}^{\prime}\right\|_{\infty}<D$ and $\left\|\Phi_{\varepsilon}^{\prime \prime}\right\|_{\infty}<D$ with a constant $D>0$ which does not depend on $\varepsilon$.

Proof. Let $\varepsilon>0$. Choose $g_{\varepsilon} \in C_{\mathrm{cpt}}^{\infty}(\mathbb{R})$ with support in the set $\left\{x \in \mathbb{R} ; R_{\varepsilon} \leq\right.$ $\left.|x| \leq S_{\varepsilon}\right\}$ such that $h_{\varepsilon}(x):=\int_{-\infty}^{x} g_{\varepsilon}(t) d t$ is equal to $h_{\varepsilon, 1}+h_{\varepsilon, 2}$, where $h_{\varepsilon, 1}$ is an everywhere non-negative bump function with support $\left[-S_{\varepsilon},-R_{\varepsilon}\right]$ and total mass $\int h_{\varepsilon, 1}(x) d x=1$ and $h_{\varepsilon, 2}$ is the negative of the reflection of $h_{\varepsilon, 1}$. Set $\Phi_{\varepsilon}(x):=\int_{-\infty}^{x} h_{\varepsilon}(t) d t$. Then (i) and (ii) obviously hold. Furthermore, we can assume that all functions $g_{\varepsilon}$ are translates of each other, and hence the same applies to all functions $h_{\varepsilon}$. This implies (iii).

Let $W$ be the set of all smooth $L^{1}$-functions with compactly supported Fourier transform.

1.1.2 Lemma. Let $f \in C_{\mathrm{cpt}}^{\infty}(\mathbb{R})$ be an smooth function with compact support. Then there exists for each $\delta>0$ a function $f_{\delta} \in W$ with the following properties (where the suprema are taken over all $x \in \mathbb{R}$ ):

(i) $\sup \left|f(x)-f_{\delta}(x)\right|<\delta$,

(ii) $\sup \left|x\left(f(x)-f_{\delta}(x)\right)\right|<\delta$,

(iii) $\sup \left|x^{2}\left(f(x)-f_{\delta}(x)\right)\right|<\delta$, and

(iv) $\left\|f_{\delta}\right\|_{\infty} \leq\|f\|_{\infty}$.

Furthermore, $\operatorname{supp}\left(\hat{f}_{\delta}\right) \subset[-C(\delta), C(\delta)]$ where $C(\delta) \rightarrow \infty$ for $\delta \rightarrow 0$. 


\section{A. Appendix}

Proof. Let $\delta>0$. Consider the Fourier transform $F:=\hat{f} \in \mathscr{S}$ of $f$. (In the following we denote the Fourier transform of a function which is denoted by a lower case letter by the corresponding upper case letter. This will simplify notation when we consider derivatives of such transforms.) Let $\varepsilon>0$ and $R_{\varepsilon}, S_{\varepsilon}>0$ and $\Phi_{\varepsilon}$ as well as $C>0$ be as in the Technical Lemma 1.1.1. Define an auxiliary function $f_{\varepsilon}$ by its Fourier transform via $F_{\varepsilon}(\xi):=\Phi_{\varepsilon}(\xi) F(\xi)$. Notice $\operatorname{supp}\left(F_{\varepsilon}\right) \subset \operatorname{supp}\left(\Phi_{\varepsilon}\right) \subset\left[-S_{\varepsilon}, S_{\varepsilon}\right]$. Thus, with $C(\varepsilon):=S_{\varepsilon}$, one has $\operatorname{supp}\left(\hat{f}_{\varepsilon}\right) \subset[-C(\varepsilon), C(\varepsilon)]$ and $C(\varepsilon) \rightarrow \infty$ for $\varepsilon \rightarrow 0$. In the end we will define $f_{\delta}$ as $f_{\varepsilon}$ for an appropriate $\varepsilon>0$, which will be such that we can prove (i) - (iii). For this we make use of the following fact: Whenever $u$ and $v$ are (suitable) functions with Fourier transforms $U$ and $V$, one has

$$
\left\|x^{k}(u(x)-v(x))\right\|_{\infty} \leq\left\|U^{(k)}(\xi)-V^{(k)}(\xi)\right\|_{1} .
$$

Choose $m \in \mathbb{N}$ with $m \geq 2$.

Ad (i): Since $F$ is a Schwartz function there exists a constant $D_{m}>0$ such that $\left|\xi^{m} F(\xi)\right| \leq D_{m}$ for each $\xi \in \mathbb{R}$. Using this we can make the following estimate:

$$
\begin{aligned}
\left\|F-F_{\varepsilon}\right\|_{1} & =\left\|\left(1-\Phi_{\varepsilon}\right) F\right\|_{1} \\
& \leq \int_{|\xi| \geq R_{\varepsilon}}|F(\xi)| d \xi \\
& \leq D_{m} \int_{|\xi| \geq R_{\varepsilon}} \frac{d \xi}{|\xi|^{m}} .
\end{aligned}
$$

This implies (i) since $R_{\varepsilon} \rightarrow \infty$ as $\varepsilon \rightarrow 0$.

Ad (ii): To begin with, one has:

$$
F^{\prime}(\xi)-F_{\varepsilon}^{\prime}(\xi)=\left(1-\Phi_{\varepsilon}(\xi)\right) F^{\prime}(\xi)+\Phi_{\varepsilon}^{\prime}(\xi) F(\xi) .
$$

Furthermore, there exists $D_{m}>0$ with $\left|\xi^{m} F(\xi)\right| \leq D_{m}$ and $\left|\xi^{m} F^{\prime}(\xi)\right| \leq D_{m}$ for each $\xi \in \mathbb{R}$. Using this we can estimate as follows:

$$
\begin{aligned}
\left\|F^{\prime}-F_{\varepsilon}^{\prime}\right\|_{1} & \leq\left\|\left(1-\Phi_{\varepsilon}\right) F^{\prime}\right\|_{1}+\left\|\Phi_{\varepsilon}^{\prime} F\right\|_{1} \\
& \leq \int_{|\xi| \geq R_{\varepsilon}}\left|F^{\prime}(\xi)\right| d \xi+C \int_{R_{\varepsilon} \leq|\xi| \leq S_{\varepsilon}}|F(\xi)| d \xi \\
& \leq D_{m} \int_{|\xi| \geq R_{\varepsilon}} \frac{d \xi}{|\xi|^{m}}+C D_{m} \int_{R_{\varepsilon} \leq|\xi| \leq S_{\varepsilon}} \frac{d \xi}{|\xi|^{m}} .
\end{aligned}
$$

This, together with the fact that $R_{\varepsilon} \rightarrow \infty$ as $\varepsilon \rightarrow 0$, implies (ii).

Ad (iii): This can be proven by the same reasoning as in (ii): First of all, one has:

$$
F^{\prime \prime}(\xi)-F_{\varepsilon}^{\prime \prime}(\xi)=\left(1-\Phi_{\varepsilon}(\xi)\right) F^{\prime \prime}(\xi)+\Phi_{\varepsilon}^{\prime \prime}(\xi) F(\xi) .
$$

Using this, we can estimate as follows:

$$
\begin{aligned}
\left\|F^{\prime \prime}-F_{\varepsilon}^{\prime \prime}\right\|_{1} & \leq\left\|\left(1-\Phi_{\varepsilon}\right) F^{\prime \prime}\right\|_{1}+\left\|\Phi_{\varepsilon}^{\prime \prime} F\right\|_{1} \\
& \leq \int_{|\xi| \geq R_{\varepsilon}}\left|F^{\prime \prime}(\xi)\right| d \xi+C \int_{R_{\varepsilon} \leq|\xi| \leq S_{\varepsilon}}|F(\xi)| d \xi .
\end{aligned}
$$




\section{A. Appendix}

Since $F$ is a Schwartz function and $R_{\varepsilon} \rightarrow \infty$ as $\varepsilon \rightarrow \infty$ this implies (iii).

Ad (iv): Let $\varphi_{\varepsilon} \in W$ be such that $\Phi_{\varepsilon}=\hat{\varphi}_{\varepsilon}$. By definition, $f_{\varepsilon}=(2 \pi)^{-1}\left(\varphi_{\varepsilon} * f\right)$. Young's inequality implies the following estimate:

$$
\left\|f_{\varepsilon}\right\|_{\infty}=\left\|\varphi_{\varepsilon} * f\right\|_{\infty} \leq\left\|\varphi_{\varepsilon}\right\|_{1}\|f\|_{\infty} .
$$

Since $\left\|\varphi_{\varepsilon}\right\|_{1} \leq\left\|\Phi_{\varepsilon}\right\|_{\infty} \leq 1$, we can conclude (iv).

1.1.3 Lemma. Let $f \in C_{\mathrm{cpt}}^{\infty}(\mathbb{R})$ be a non-negative, compactly supported, smooth function. Then there exists for each $\varepsilon>0$ a function $f_{\varepsilon} \in C^{\infty}(\mathbb{R})$ with the following properties:

(i) $f_{\varepsilon} \geq 0$ and the square-root $g_{\varepsilon}$ of $f_{\varepsilon}$ belongs to $W$,

(ii) $\sup \left|f(x)-f_{\varepsilon}(x)\right|<\varepsilon$,

(iii) $\sup \left|x\left(f(x)-f_{\varepsilon}(x)\right)\right|<\varepsilon$ and

(iv) $\sup \left|x^{2}\left(f(x)-f_{\varepsilon}(x)\right)\right|<\varepsilon$.

Furthermore, $\operatorname{supp}\left(f_{\varepsilon}\right) \subset[-C(\varepsilon), C(\varepsilon)]$ where $C(\varepsilon) \rightarrow \infty$ for $\varepsilon \rightarrow 0$.

Proof. Set $F:=\sqrt{f}$. Because this function is continuous and vanishes outside of a compact set, there exists by [30, Thm. 2.3, 13.2, p. 357] and [30, Thm. $3.1,13.3$, p. 359] a smooth function $G$ which is uniformly as close to $F$ as one wants. By Lemma 1.1.2, $G$ admits a decomposition $G=G_{\delta}+R_{\delta}$ with $G_{\delta} \in W$ such that

$$
\sup _{x \in \mathbb{R}}\left|x^{k} R_{\delta}(x)\right|<\delta \quad, \quad k=0,1,2
$$

as well as $\left\|G_{\delta}\right\|_{\infty} \leq\|G\|$. Furthermore, $\operatorname{supp}\left(G_{\delta}\right) \subset[-C(\delta), C(\delta)]$ where $C(\delta) \rightarrow \infty$ for $\delta \rightarrow 0$. One has

$$
G^{2}=G_{\delta}^{2}+2 G_{\delta} R_{\delta}+R_{\delta}^{2} .
$$

Set $f_{\delta}:=G_{\delta}^{2}$ and $r_{\delta}:=2 G_{\delta} R_{\delta}+R_{\delta}^{2}$. Then (i) holds.

Ad (ii) - (iv): For each $k=0,1,2$ one has

$$
\begin{aligned}
\left|x^{k}\left(f-f_{\delta}\right)(x)\right| & \left.=\mid x^{k}\left(\sqrt{f}-\sqrt{f_{\delta}}\right)(x)\left(\sqrt{f}+\sqrt{f_{\delta}}\right)(x)\right) \mid \\
& \leq\left|x^{k}\left(F-G_{\delta}\right)(x)\right|\left\|F+G_{\delta}\right\|_{\infty} \\
& \leq\left(\left|x^{k}(F-G)(x)\right|+\left|x^{k}\left(G-G_{\delta}\right)(x)\right|\right)\left\|F+G_{\delta}\right\|_{\infty} \\
& \leq\left(\left|x^{k}(F-G)(x)\right|+\left|x^{k} R_{\delta}(x)\right|\right)\left(\|F\|_{\infty}+\left\|G_{\delta}\right\|_{\infty}\right)
\end{aligned}
$$

This implies the claim. 


\section{A. Appendix}

\subsection{More detailed estimates}

For equation (2): Set $g(t):=t^{2}$ and $h(t):=f(t) g(t)=t^{2} f(t)$. With this one obtains using the Cauchy-Schwarz inequality and Theorem 1.3.3 the following estimates

$$
\begin{aligned}
\left|\left\langle D^{2} f(D) u, u\right\rangle\right| & =|\langle h(D) u, u\rangle| \\
& =|\langle(f \cdot g)(D) u, u\rangle| \\
& =|\langle(f(D) \circ g(D)) u, u\rangle| \\
& =|\langle(\varphi(D) \circ \varphi(D) \circ g(D)) u, u\rangle| \\
& =|\langle(\varphi(D) \circ g(D)) u, \varphi(D) u\rangle| \\
& =|\langle(g(D) \circ \varphi(D)) u, \varphi(D) u\rangle| \\
& =|\langle g(D)(\varphi(D) u), \varphi(D) u\rangle| \\
& \leq\|g(D)(\varphi(D) u)\|\|\varphi(D) u\| \\
& \leq\|g(D)\|\|\varphi(D) u\|^{2} \\
& \leq\|g\|_{\infty}\|\varphi(D) u\|^{2} \\
& \leq \alpha^{2}\|\varphi(D) u\|^{2} .
\end{aligned}
$$

For equation (3): Set $g(t):=t^{2}$ and $h(t)=g(t) f_{\delta}(t)$. Then one obtains using $\|h\|_{\infty} \leq \delta$ the estimate

$$
\begin{aligned}
\left|\left\langle D^{2} f_{\delta}(D) u, u\right\rangle\right| & =\left|\left\langle\left(g \cdot f_{\delta}\right)(D) u, u\right\rangle\right| \\
& =|\langle h(D) u, u\rangle| \\
& \leq\|h(D) u\|\|u\| \\
& \leq\|h\|_{\infty}\|u\|^{2} \\
& \leq \delta\|u\|^{2} .
\end{aligned}
$$




\section{Bibliography}

[1] Bernd Ammann and Emmanuel Humbert. The spinorial $\tau$-invariant and 0-dimensional surgery. J. Reine Angew. Math., 624:27-50, 2008.

[2] Helga Baum. Complete Riemannian manifolds with imaginary Killing spinors. Ann. Global Anal. Geom., 7(3):205-226, 1989.

[3] Bruce Blackadar. K-theory for operator algebras, volume 5 of Mathematical Sciences Research Institute Publications. Cambridge University Press, Cambridge, second edition, 1998.

[4] J. Bland and M. Kalka. Complete metrics of negative scalar curvature on noncompact manifolds. In Nonlinear problems in geometry (Mobile, Ala., 1985), volume 51 of Contemp. Math., pages 31-35. Amer. Math. Soc., Providence, RI, 1986.

[5] Jonathan Block and Shmuel Weinberger. Arithmetic manifolds of positive scalar curvature. J. Differential Geom., 52(2):375-406, 1999.

[6] Ulrich Bunke. A $K$-theoretic relative index theorem and Callias-type Dirac operators. Math. Ann., 303(2):241-279, 1995.

[7] Stanley Chang. Positive scalar curvature of totally nonspin manifolds. Proc. Amer. Math. Soc., 138(5):1621-1632, 2010.

[8] Stanley Chang, Shmuel Weinberger, and Guoliang Yu. Taming 3-manifolds using scalar curvature. Geom. Dedicata, 148:3-14, 2010.

[9] Jeff Cheeger and David G. Ebin. Comparison theorems in Riemannian geometry. AMS Chelsea Publishing, Providence, RI, 2008.

[10] Jean Dieudonné. A history of algebraic and differential topology 19001960. Modern Birkhäuser Classics. Birkhäuser Boston Inc., Boston, MA, 2009.

[11] Nelson Dunford and Jacob T. Schwartz. Linear operators. Part II. Wiley Classics Library. John Wiley \& Sons Inc., New York, 1988.

[12] Thomas Friedrich. Dirac operators in Riemannian geometry, volume 25 of Graduate Studies in Mathematics. American Mathematical Society, Providence, RI, 2000.

[13] Sylvestre Gallot, Dominique Hulin, and Jacques Lafontaine. Riemannian geometry. Universitext. Springer-Verlag, Berlin, third edition, 2004. 
[14] Nicolas Ginoux. The Dirac spectrum, volume 1976 of Lecture Notes in Mathematics. Springer-Verlag, Berlin, 2009.

[15] Mikhael Gromov and H. Blaine Lawson, Jr. The classification of simply connected manifolds of positive scalar curvature. Ann. of Math. (2), 111(3):423-434, 1980.

[16] Mikhael Gromov and H. Blaine Lawson jun. Positive scalar curvature and the Dirac operator on complete Riemannian manifolds. Publ. Math., Inst. Hautes Étud. Sci., 58:83-196, 1983.

[17] Bernhard Hanke and Thomas Schick. Enlargeability and index theory. $J$. Differential Geom., 74(2):293-320, 2006.

[18] Nigel Higson. $K$-homology and operators on non-compact manifolds. Preprint availabe from Higson's webpage.

[19] Nigel Higson. A note on the cobordism invariance of the index. Topology, 30(3):439-443, 1991.

[20] Nigel Higson, Erik Kjær Pedersen, and John Roe. $C^{*}$-algebras and controlled topology. K-Theory, 11(3):209-239, 1997.

[21] Nigel Higson and John Roe. Analytic K-homology. Oxford Mathematical Monographs. Oxford University Press, Oxford, 2000.

[22] Nigel Higson, John Roe, and Guoliang Yu. A coarse Mayer-Vietoris principle. Math. Proc. Cambridge Philos. Soc., 114(1):85-97, 1993.

[23] Kjeld Knudsen Jensen and Klaus Thomsen. Elements of KK-theory. Mathematics: Theory \& Applications. Birkhäuser Boston Inc., Boston, MA, 1991.

[24] Michael Joachim and Thomas Schick. Positive and negative results concerning the Gromov-Lawson-Rosenberg conjecture. In Geometry and topology: Aarhus (1998), volume 258 of Contemp. Math., pages 213-226. Amer. Math. Soc., Providence, RI, 2000.

[25] Jerry L. Kazdan and F. W. Warner. Existence and conformal deformation of metrics with prescribed Gaussian and scalar curvatures. Ann. of Math. (2), 101:317-331, 1975.

[26] Masoud Khalkhali. Basic noncommutative geometry. EMS Series of Lectures in Mathematics. European Mathematical Society (EMS), Zürich, 2009 .

[27] Antoni A. Kosinski. Differential manifolds, volume 138 of Pure and Applied Mathematics. Academic Press Inc., Boston, MA, 1993.

[28] Dan Kucerovsky. Functional calculus and representations of $C_{0}(\mathbb{C})$ on a Hilbert module. Q. J. Math., 53(4):467-477, 2002. 
Bibliography

[29] E. Christopher Lance. Hilbert $C^{*}$-modules, volume 210 of London Mathematical Society Lecture Note Series. Cambridge University Press, Cambridge, 1995.

[30] Serge Lang. Real analysis. Addison-Wesley Publishing Company Advanced Book Program, Reading, MA, second edition, 1983.

[31] H. Blaine Lawson, Jr. and Marie-Louise Michelsohn. Spin geometry, volume 38 of Princeton Mathematical Series. Princeton University Press, Princeton, NJ, 1989.

[32] Varghese Mathai. Nonnegative scalar curvature. Ann. Global Anal. Geom., 10(2):103-123, 1992.

[33] Gerard J. Murphy. $C^{*}$-algebras and operator theory. Academic Press Inc., Boston, MA, 1990.

[34] Vladimir E. Nazaikinskii, Anton Yu. Savin, and Boris Yu. Sternin. Elliptic theory and noncommutative geometry, volume 183 of Operator Theory: Advances and Applications. Birkhäuser Verlag, Basel, 2008.

[35] John Roe. Partitioning non-compact manifolds and the dual toeplitz problem. Operator algebras and application. Vol. 1: Structure theory; K-theory, geometry and topology, Pap. UK-US Jt. Semin., Warwick/UK 1987, Lond. Math. Soc. Lect. Note Ser. 135, 187-228, 1988.

[36] John Roe. Exotic cohomology and index theory. Bull. Amer. Math. Soc. (N.S.), 23(2):447-453, 1990.

[37] John Roe. Coarse Cohomology and Index Theory on Complete Riemannian Manifolds. Mem. Amer. Math. Soc., 104(497):x+90, 1993.

[38] John Roe. Index theory, coarse geometry, and topology of manifolds, volume 90 of CBMS Regional Conference Series in Mathematics. Published for the Conference Board of the Mathematical Sciences, Washington, DC, 1996.

[39] John Roe. Comparing analytic assembly maps. Q. J. Math., 53(2):241-248, 2002 .

[40] John Roe. Lectures on coarse geometry, volume 31 of University Lecture Series. American Mathematical Society, Providence, RI, 2003.

[41] Thomas Schick. A counterexample to the (unstable) Gromov-LawsonRosenberg conjecture. Topology, 37(6):1165-1168, 1998.

[42] Claude Schochet. Topological methods for $C^{*}$-algebras. II. Geometric resolutions and the Künneth formula. Pacific J. Math., 98(2):443-458, 1982.

[43] Herbert Schröder. $K$-theory for real $C^{*}$-algebras and applications, volume 290 of Pitman Research Notes in Mathematics Series. Longman Scientific \& Technical, Harlow, 1993. 


\section{Bibliography}

[44] Yu. P. Solovyov and E. V. Troitsky. $C^{*}$-algebras and elliptic operators in differential topology, volume 192 of Translations of Mathematical Monographs. American Mathematical Society, Providence, RI, 2001.

[45] Ralph Stöcker and Heiner Zieschang. Algebraische Topologie. Mathematische Leitfäden. B. G. Teubner, Stuttgart, second edition, 1994.

[46] Stephan Stolz. Concordance classes of positive scalar curvature metrics. Preprint availabe from Stolz' webpage.

[47] Tammo tom Dieck. Algebraic topology. EMS Textbooks in Mathematics. European Mathematical Society (EMS), Zürich, 2008.

[48] Niels Erik Wegge-Olsen. K-theory and $C^{*}$-algebras. Oxford Science Publications. The Clarendon Press Oxford University Press, New York, 1993.

[49] Guoliang Yu. $K$-theoretic indices of Dirac type operators on complete manifolds and the Roe algebra. K-Theory, 11(1):1-15, 1997.

[50] Mostafa Esfahani Zadeh. A note on some classical results of GromovLawson. Available from arxiv.org under arXiv:0912.3352.

[51] Mostafa Esfahani Zadeh. Index theory and partitioning by enlargeable hypersurfaces. J. Noncommut. Geom., 4(3):459-473, 2010. 